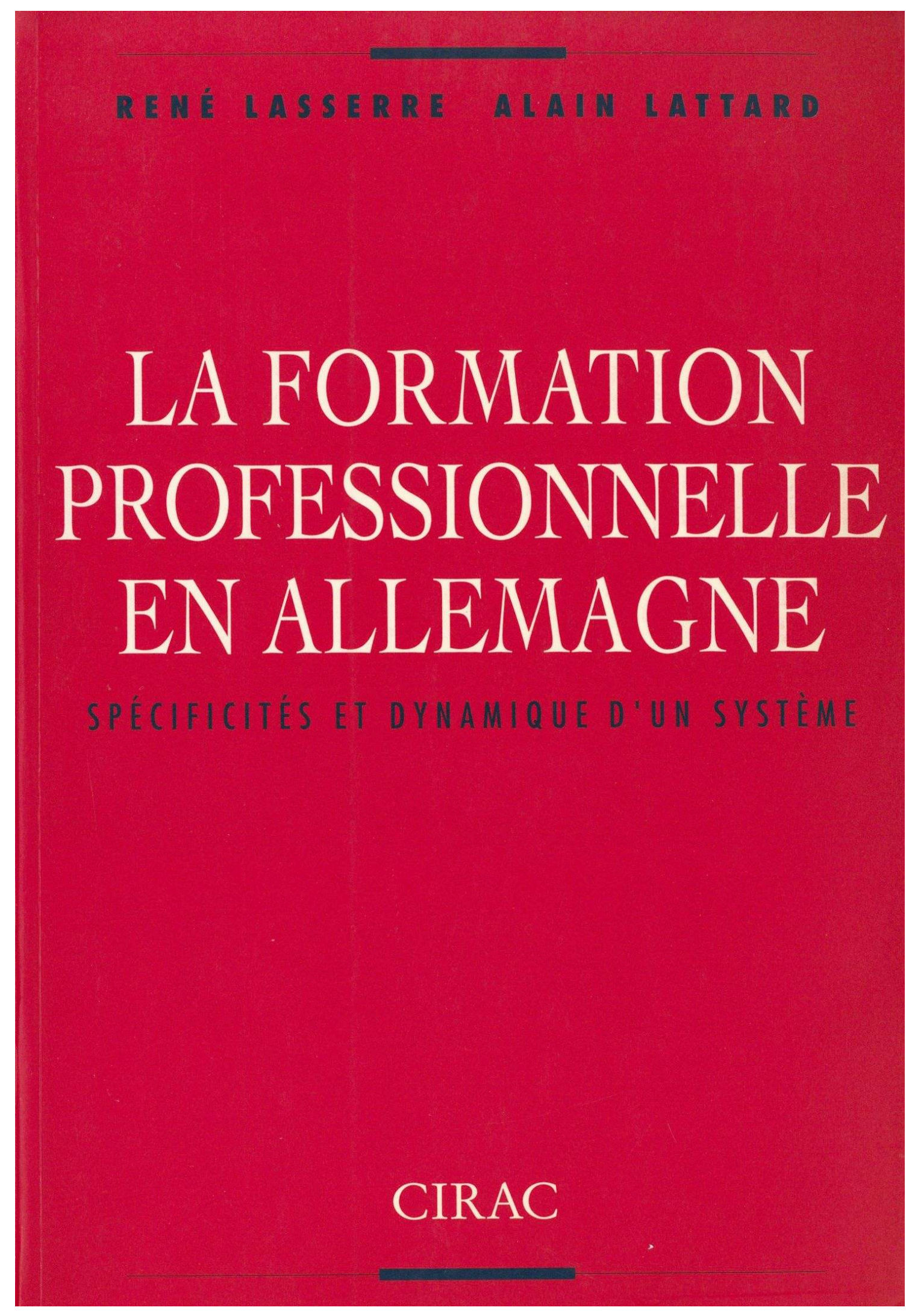




\section{La formation professionnelle en Allemagne}

Spécificités et dynamique d'un système

\section{René Lasserre et Alain Lattard}

DOI : 10.4000/books.cirac. 896

Éditeur : IFAEE

Année d'édition : 1993

Date de mise en ligne : 13 décembre 2017

Collection : Travaux et documents du CIRAC

ISBN électronique : 9782905518514

\section{opanatition}

http://books.openedition.org

Édition imprimée

ISBN : 9782905518248

Nombre de pages : 128

\section{Référence électronique}

LASSERRE, René ; LATTARD, Alain. La formation professionnelle en Allemagne : Spécificités et dynamique d'un système. Nouvelle édition [en ligne]. Cergy-Pontoise : IFAEE, 1993 (généré le 02 octobre 2020). Disponible sur Internet : <http://books.openedition.org/cirac/896>. ISBN : 9782905518514. DOI : https://doi.org/10.4000/books.cirac.896.

Ce document a été généré automatiquement le 2 octobre 2020. II est issu d'une numérisation par reconnaissance optique de caractères.

(c) IFAEE, 1993

Conditions d'utilisation:

http://www.openedition.org/6540 
Les politiques de formation sont confrontées à deux défis majeurs : répondre aux besoins de qualification des entreprises et assurer l'insertion professionnelle des jeunes.

Parmi les grands pays industriels, l'Allemagne dispose d'un système de formation professionnelle qui, de ce double point de vue, compte indiscutablement parmi les plus performants. Pour l'essentiel, cette réussite repose sur une forte implication de l'entreprise dans la formation initiale. C'est ce que montre la présente étude, qui dresse un bilan synthétique de « l'alternance à l'allemande ».

Après un bref rappel historique et une description du cadre institutionnel, René Lasserre et Alain Lattard évaluent la qualité des formations dispensées, la question de leur coût pour l'entreprise et celle de leur efficacité en termes d'emploi. Ils mettent en évidence les capacités d'adaptation du système allemand, sans pour autant ignorer ses difficultés face à l'évolution de la demande d'éducation.

\section{RENÉ LASSERRE}

Docteur en Études allemandes contemporaines et spécialiste des questions économiques et sociales allemandes, il dirige le Centre d'information et de recherche sur l'Allemagne contemporaine. Il est également maître de conférences à l'université de Paris III et professeur à l'Institut d'études politiques de Paris.

\section{ALAIN LATTARD}

Docteur en Études allemandes contemporaines, il est maître de conférences à l'université de Paris III et chercheur associé au Centre d'information et de recherche sur l'Allemagne contemporaine. Spécialiste des relations industrielles et sociales en Allemagne, il est auteur notamment de La Réduction du temps de travail en Allemagne fédérale, Paris, 1987. 
SOMMAIRE

\section{Introduction}

René Lasserre et Alain Lattard

Introduction

I. Genèse du système dual : une tradition ancienne, mais une stabilisation récente

Une continuité séculaire aux cheminements complexes

Une stabilisation tardive et tout d'abord précaire

II. L'apprentissage dual : élément central d'un système diversifié de formation professionnelle

La prépondérance du système dual

Les formations professionnelles scolaires à plein temps

\section{L'entreprise formatrice au centre d'un système de régulation concertée}

Un système de régulation tripartite à plusieurs niveaux

L'école professionnelle, maillon faible du système

Prépondérance de l'entreprise et consensus social

IV. Une offre de formation différenciée, mais de qualité

Une offre de formation différenciée

Les règlements de formation (Ausbildungsordnungen) : vecteurs de diffusion d'une culture professionnelle

L'évolution vers la polyvalence des qualifications

\section{Financement et coût de la formation dans le système dual}

Répartition de l'effort de financement

La contribution de l'Etat

Les dépenses de formation de l'entreprise

La prestation productive de l'apprenti

La rentabilité indirecte de l'investissement-formation initial

VI. Le système dual entre l'école et la vie professionnelle

Un accès ouvert et un faible degré d'exclusion

La formation duale : un atout pour l'insertion professionnelle

\section{Bilan et perspectives}

Les vertus de l'alternance

Le tribut de la modernisation

Les problèmes de recrutement

Orientation bibliographique

Adresses utiles

Liste des tableaux et figures

Index thématique 


\title{
Introduction
}

\author{
René Lasserre et Alain Lattard
}

\section{Introduction}

1 La présente étude a pour objet de dresser un bilan synthétique du système allemand de formation professionnelle initiale. Le propos n'est pas d'en livrer une description complète et détaillée, mais d'analyser dans un premier temps comment le principe central de l'alternance, c'est-à-dire la conjonction de l'école et de l'entreprise comme lieux de formation - ce que les Allemands désignent sous le terme de "système dual" - a été mis en œuvre en Allemagne et comment il se traduit concrètement dans l'architecture et le fonctionnement du système.

2 Dans un deuxième temps, l'étude examine les performances du système dual au regard de trois critères essentiels : celui de la qualité des formations dispensées, celui de son coût financier et de sa rentabilité économique, celui de son efficacité "sociale" du point de vue des perspectives d'insertion professionnelle offertes aux jeunes Allemands.

3 Enfin, dans une dernière partie évaluative et prospective, l'étude tente de dresser un bilan du système dual allemand en mettant en évidence non seulement ses avantages, mais aussi ses points faibles. Elle s'interroge en particulier sur l'attractivité de cette voie de formation jusqu'alors dominante et sur les perspectives d'évolution du "modèle". Loin d'être dépassé, comme on l'affirme parfois un peu vite, le système dual allemand est néanmoins confronté au processus d'élévation du niveau d'instruction générale que connaissent tous les pays industriels développés.

Dans son approche évaluative, la présente étude n'a pas l'ambition de comparer les performances du système allemand avec celles du système français ou d'autres systèmes étrangers. Outre qu'une telle analyse est difficile à mener, car elle impliquerait une prise en compte soigneusement pondérée d'éléments contextuels spécifiques (par exemple des données démographiques pour ce qui est de l'insertion professionnelle des jeunes), elle aurait largement dépassé le cadre de cet ouvrage. Notre objectif était bien davantage de mettre en évidence les spécificités du système dual et d'en expliquer la logique et la dynamique internes. Ce n'est que 
subsidiairement, et par rapport au principe central de l'alternance, que nous avons cherché à dégager quelques enseignements pour la réflexion actuellement en cours en France.

5 L'analyse porte essentiellement sur le système de formation tel qu'il fonctionne dans les Länder de l'Ouest et n'intègre pas la problématique particulière de son extension progressive dans les nouveaux Länder de l'Est. Même si cette extension se heurte à de nombreuses difficultés, en particulier à une offre de places d'apprentissage en forte régression dans les entreprises est-allemandes, elle n'est pas de nature à bouleverser l'architecture générale du système tel qu'il fonctionne à l'Ouest. La structure plus traditionnelle de la demande d'éducation dans les nouveaux Länder, avec une part plus importante de jeunes choisissant le système dual peut même atténuer pour un temps les problèmes de recrutement.

6 Notre étude s'appuie non seulement sur l'exploitation des données, expertises, ouvrages et articles de référence publiés dans la période récente, mais également sur un travail d'enquête. Elle nous a conduit à nous entretenir directement avec différents responsables institutionnels et spécialistes allemands dans le domaine de la formation. Mais pour l'essentiel, nous nous sommes appuyés sur une enquête de terrain auprès des services et responsables de formation d'une douzaine d'entreprises allemandes de différente taille et de différents secteurs: Bayer AG Leverkusen, Dr. Paul Lohmann GmbH KG Emmerthal (chimie), Robert Bosch GmbH Stuttgart, Siemens Berlin (électrotechnique), Volkswagenwerk Wolfsburg (automobile), Fleischhauer Cologne (concessionnaire et réparation automobile), Trumpf $\mathrm{GmbH} \&$ Co Ditzingen (construction de machines), Sennheiser Electronic KG Wedemark (appareils de transmission et de mesure sonores), Phywe Systeme GmbH Göttingen (appareils et fournitures de laboratoires), Deutsche Bank AG Francfort (banque), Deutsche Krankenversicherung AG Cologne (compagnie privée d'assurance-maladie complémentaire), Gerling-Konzern Cologne (assurance industrielle). Cette enquête a été complétée par la visite d'une école professionnelle (Berufsschulzentrum Leonberg) et d'un centre de formation inter-entreprises (Gemeinschaftsausbildungsstätte RuithPlochingen) dans le Land de Bade-Wurtemberg.

7 Nous remercions très vivement les personnalités que nous avons rencontrées pour l'accueil chaleureux qu'elles nous ont réservé et pour leur active et précieuse collaboration à la réalisation de cette étude. 


\section{Genèse du système dual : une tradition ancienne, mais une stabilisation récente}

1 Il est rare que le succès d'un produit d'exportation ne soit pas fondé sur une réussite à l'intérieur des frontières. Aussi a-t-on tendance à penser automatiquement que le système dual de formation professionnelle, facteur de compétitivité que ses concurrents envient à l'Allemagne, a été naturellement accepté dans le pays où il s'est constitué. Un bref rappel historique aide à relativiser cet a priori simplificateur. En effet, si le système dual relève d'une longue tradition, des origines à l'état actuel, la filiation est complexe et parfois paradoxale; c'est dire aussi que le modèle s'est construit lentement, que des conjonctures difficiles ont bien failli l'emporter, mais que survivant à ces difficultés successives, le principe de formation professionnelle en alternance a fait preuve d'une grande capacité d'adaptation.

\section{Une continuité séculaire aux cheminements complexes}

2 Dans la lente élaboration du modèle allemand d'enseignement professionnel, qui s'amorce à la fin du XXe siècle, on peut déceler trois lignes de continuité1.

3 A l'origine, on trouve la politique menée au tournant du siècle dans un contexte de crise économique et de montée de la social-démocratie par les très conservateurs gouvernements de l'Empire. Pour s'assurer le soutien des classes moyennes, ceux-ci amendent la législation qui, généralisant la liberté d'entreprise (Gewerbeordnung de 1869 pour la confédération d'Allemagne du Nord, puis de 1871 pour l'ensemble du Reich), avait pratiquement aboli toute réglementation de l'apprentissage: tout entrepreneur pouvait recruter des apprentis dès lors qu'il jouissait de ses droits civiques, et la formation qu'il donnait n'était que très peu contrôlée et sanctionnée par aucun examen. Par touches successives, des modifications de la Gewerbeordnung qui interviennent entre 1881 et 1908 restaurent d'une part des formes d'organisations artisanales de branche (Innungen), puis les chambres d'artisanat (Handwerkskammern): 
le statut de "corporation de droit public", qu'elles obtiennent fondent la tradition depuis lors jamais interrompue d'une gestion de la formation en entreprise par des organisations de l'économie dotées de l'autonomie administrative; d'autre part, le droit de former des apprentis est désormais réservé aux seuls maîtres-artisans.

De telles mesures apportent au mouvement artisanal une triple satisfaction, tant idéologique, sociale, qu'économique: elles contribuent à la fois à réhabiliter une tradition professionnelle qui se sent menacée par l'expansion de la production industrielle de masse, à justifier le discours sur l'artisanat, éducateur d'une jeunesse que l'industrie déracine et expose à la "contamination socialiste", enfin à stabiliser la situation économique du secteur, en donnant un privilège aux maîtres, bien que ceux-ci ne recouvrent pas le droit exclusif de fonder une entreprise.

5 Si cette institutionnalisation de la formation en entreprise signifie bien l'avènement officiel de l'apprentissage dual, elle marque une origine paradoxale, dans la mesure où est privilégiée la tradition artisanale, alors même que le pays connaît son véritable démarrage industriel. La contradiction est pourtant moins forte qu'il n'y parait: d'abord, parce que l'artisanat est alors (et restera longtemps) le principal pourvoyeur de main-d'œuvre de l'industrie ; ensuite, parce qu'une telle évolution n'empêche pas les initiatives de l'industrie.

6 Pour le gros des emplois, peu à peu déqualifiés par la mécanisation et la parcellisation du travail, cette dernière ne pratique qu'une formation sommaire sur le tas. Mais les emplois qualifiés qui subsistent requièrent des savoir-faire que les ouvriers issus de la formation artisanale possèdent mal. Non seulement l'éthique professionnelle qui leur est inculquée est peu compatible avec la production de masse, mais les connaissances techniques transmises sont souvent trop prisonnières de la tradition, à moins qu'elles soient franchement insuffisantes, lorsque le maître-artisan est plus préoccupé de recruter une main-d'œuvre bon marché que de bien former le petit nombre de jeunes qu'il compte recruter ensuite (Lehrlingszüchterei). C'est pourquoi dès la dernière décennie du siècle, les grandes entreprises des secteurs de pointe créent les premiers ateliers d'apprentissage.

7 Même si l'apprentissage industriel est encore à l'époque quantitativement insignifiant, on peut voir dans cette initiative la deuxième racine du système dual, à moins qu'il ne s'agisse de son seul acte de naissance légitime, car des deux évolutions décrites, c'est elle qui est la plus porteuse d'avenir. L'industrie marque en effet par là sa volonté précoce de s'impliquer dans la solution d'un problème toujours actuel, la meilleure adaptation possible de la qualification aux besoins, tels qu'ils résultent du choix de l'organisation du travail et des exigences de l'évolution technique. Il reste que le trait le plus spécifique du système dual, son enracinement dans l'entreprise, est le produit d'un singulier mélange de conservatisme et de modernité.

8 Le deuxième pilier du système dual, l'enseignement scolaire à temps partiel, s'édifie également à la même époque, lorsque les "écoles de perfectionnement" (Fortbildungsschulen) existant depuis la fin du XVIII ${ }^{\mathrm{e}}$ siècle et initialement destinées à compléter l'enseignement de l'école primaire pour les jeunes travailleurs, se multiplient en intégrant progressivement des éléments d'enseignement professionnel. A ces deux évolutions institutionnelles, sur lesquelles se fonde le système dual, s'ajoute une troisième ligne de continuité, qui en assied la légitimité, l'attitude des syndicats de salariés, faite à la fois d'adhésion et de critique. Il y a certes chez eux la volonté de ne pas laisser l'apprentissage à la seule responsabilité du patronat, ce qui les conduit à 
réclamer à partir des années 20 une loi instituant un contrôle public sur la formation professionnelle et une participation de leurs représentants aux organismes de surveillance correspondants. L'aboutissement de cette revendication en... 1969 contribuera à stabiliser le système.

9 Cependant, même si leur revendication reste longtemps ignorée, les organisations de salariés ne condamnent pas pour autant l'apprentissage en entreprise en tant que tel. C'est d'abord que, compte tenu du fédéralisme et de l'évolution politique passée et présente, l'école publique ne leur semble pas, comme ce peut être le cas en France, constituer une alternative à la fois cohérente et politiquement progressiste. Mais c'est aussi que, préoccupés de maintenir une formation ancrée dans la pratique, ils savent apprécier les avantages d'une primauté de l'entreprise comme lieu de formation.

\section{Une stabilisation tardive et tout d'abord précaire}

10 La dualité que nous connaissons aujourd'hui existait donc déjà il $\mathrm{y}$ a près d'un siècle, mais le système ne se consolide que lentement, et non sans connaître des changements substantiels.

11 Un élément essentiel est l'effort de l'industrie pour infléchir le modèle en fonction de ses besoins par la systématisation et la standardisation des formations. La définition de profils professionnels progresse dans les années 20, mais n'est généralisée et sanctionnée par le droit de faire passer des examens industriels spécifiques devant les chambres de commerce et d'industrie qu'à partir de la deuxième moitié des années 30 , donc sous le Troisième Reich.

De même, l'organisation de l'enseignement scolaire se renforce sous la république de Weimar (c'est à partir des années 20 qu'on emploie le terme de Berufsschulen). Mais son développement s'opère très inégalement par suite du fédéralisme éducatif. Il est en outre bientôt enrayé par les difficultés budgétaires consécutives à la crise économique. L'industrie en tire argument pour défendre le principe d'un enseignement scolaire sous la tutelle exclusive de l'entreprise (Werkschule). Après bien des efforts en ce sens, elle capitulera elle-aussi devant les contraintes de la crise, mais surtout face à la volonté du régime hitlérien, qui, pour des raisons idéologiques évidentes, renforce et centralise cette branche nouvelle du système scolaire afin de mieux la dominer. Là encore, c'est largement par les hasards de l'Histoire que la dualité école publique-entreprise, formule traditionnelle de l'artisanat, s'impose finalement à l'industrie. Et à cinquante ans d'intervalle, de l'Empire wilhelminien à la période hitlérienne, se répète donc le paradoxe d'une modernisation décisive due à l'action d'un régime socialement régressif.

Telle qu'elle fonctionne aujourd'hui en RFA, la formation professionnelle duale a été juridiquement formalisée depuis à peine plus de vingt ans. Auparavant, les mécanismes de formation reposaient sur des normes dispersées et disparates, car noyées dans les différents textes réglementant l'activité économique des entreprises, loi sur l'activité industrielle (Gewerbeordnung), code du commerce (Handelsgesetzbuch) et loi sur l'activité artisanale (Handwerksordnung).

14 S'il faut attendre aussi longtemps pour que soit adopté un texte spécifique, c'est que l'élaboration d'une législation cohérente, amorcée dès la fin des années 50 , se heurte à un conflit d'intérêt majeur : les syndicats et le parti social-démocrate ne veulent pas se 
contenter d'unifier, ils souhaitent aussi amender le droit, notamment en introduisant la cogestion et un contrôle public de la qualité des formateurs comme des formations. Le patronat, qui tient à préserver la compétence exclusive de ses organes de gestion corporative (chambres de commerce et d'industrie, chambres de l'artisanat) en matière d'enseignement professionnel, s'oppose à l'institution d'une "organisation centraliste et bureaucratique"2, soutenu en cela par l'aile droite du parti libéral et l'union démocrate-chrétienne.

La loi sur la formation professionnelle de 1996 (Berufsbildungsgesetz - BBiG) En matière de formation professionnelle duale, la loi sur la formation professionnelle (Berufsbildungsgesetz) du 14 août 1969 occupe une place centrale. Elle a en effet rendue cohérente une législation qui reste nécessairement plurielle, compte tenu du fédéralisme éducatif. En effet, si les écoles professionnelles (Berufsschulen) ressortent exclusivement à la législation des Länder, l'apprentissage en entreprise, qui relève du droit économique et du droit du travail, peut en revanche faire l'objet d'une loi fédérale. C'est à partir de ce domaine que le texte de 1969 a unifié de manière significative le système.

Les changements concernent d'une part le système institutionnel qui régit l'apprentissage en entreprise. Les organes d'administration autonome (Selbstverwaltung) de l'économie (chambres de commerce, des métiers ou d'agriculture, selon le secteur) restent chargés du contrôle des entreprises. Mais cette fonction, qui leur incombe en tant que collectivités de droit public, doit être exercée selon des règles plus contraignantes qu'auparavant et semblables sur tout le territoire fédéral. En outre, aux termes de la loi, les jeunes de moins de 18 ans ne peuvent être formés que dans des professions agréées (anerkannte Berufe), c'est-àdire ayant fait l'objet d'un décret promulgué conjointement par le ministère fédéral de la Formation et de la Science et le ministère de tutelle (économie ou agriculture selon le domaine de formation) qui précise la dénomination de la profession, la durée, le contenu et le déroulement de la formation, ainsi que la teneur de l'examen final. D'autre part, le législateur est intervenu dans la sphère de droit privé dont relève le contrat d'apprentissage, qui est désormais standardisé. Enfin, le BBiG a institué des commissions de formation professionnelle (Berufsbildungsausschüsse), composées à parts égales de représentants des employeurs, des syndicats et des professeurs d'écoles professionnelles (ces derniers ne prenant toutefois pas part aux votes). De telles commissions fonctionnent dans les chambres économiques, où elles débattent de toutes les questions ayant trait à la formation professionnelle et adoptent les règlements nécessaires à sa mise en œuvre, notamment le règlement d'examen. Elles existent également auprès des gouvernements régionaux (Landesausschu $\beta$ ), qu'elles conseillent, veillant en particulier à la bonne coordination de la politique scolaire avec l'apprentissage en entreprise.

La formation professionnelle duale est également concernée par certaines clauses du code de l'artisanat et de la loi sur les chambres de commerce et d'industrie, ainsi que, plus accessoirement, par la loi sur la promotion du travail, la loi sur la protection du travail des jeunes et la loi sur l'organisation interne des entreprises. Dans la mesure où il s'agissait de textes antérieurs, le texte du 14 août 1969 en a harmonisé les dispositions. 
La loi votée finalement en 1969 sous le gouvernement de grande coalition (CDU/SPD) ne représente que le plus petit dénominateur commun de ces deux positions fortement divergentes. Pour l'essentiel, le texte rassemble et harmonise les normes préexistantes, ce qui satisfait plutôt le camp patronal. Il introduit cependant quelques éléments nouveaux, d'essence à la fois unificatrice et cogestionnaire, allant donc dans le sens des revendications syndicales, avec la création de commissions de formation professionnelle (Berufsbildungsausschüsse) où sont représentées les organisations de salariés tant au niveau fédéral, régional qu'au sein des chambres consulaires.

Si on peut affirmer avec deux décennies de recul que ces innovations ont finalement contribué à stabiliser le système, elles n'ont, dans l'immédiat, certes pas suffi à calmer les esprits. Pour en comprendre les raisons, il faut rappeler que la discussion sur une nouvelle loi de formation professionnelle s'inscrit à partir du milieu des années 60 dans un débat plus vaste portant sur la réforme globale du système éducatif. Deux formules marquantes, celle du sociologue Georg Picht, dénonçant l'insuffisance de bacheliers et d'étudiants comme une "catastrophe éducative" qui risque à terme d'entraîner le déclin économique de l'Allemagne et celle de son collègue Ralf Dahrendorf, qualifiant la formation générale de "droit du citoyen", suffiront ici à en suggérer l'ampleur.

Or, à peine quelques mois après l'adoption de la loi sur la formation professionnelle, la venue au pouvoir du gouvernement de petite coalition (SPD/FDP), dirigé par Willy Brandt, ouvre une phase de réformisme éducatif sans précédent. Le compromis de la loi de 1969, difficilement acquis, se trouve alors mis en cause à deux reprises.

Dans le cadre d'une série de réformes ayant pour objectif, en aval, l'ouverture et l'expansion de l'université et, en amont, la création d'un tronc commun dans le secondaire (l'école "globale" - Gesamtschule) pour lutter contre la sélection sociale, le gouvernement souhaite redéfinir la place de la formation professionnelle. Des divers projets avancés dans le camp de la majorité, le plus ambitieux vise à intégrer l'apprentissage dans le second cycle réformé de l'enseignement général. Il s'agit essentiellement de donner davantage de place à la pédagogie, aux savoirs non strictement professionnels et de réduire les différences de niveau, existant d'une branche ou d'une entreprise à l'autre. A cet égard, les réformateurs se sentent confortés par des mouvements de protestation des apprentis au début des années 70 qui, pour être ponctuels, manifestent de façon spectaculaire la négligence ou les abus de certains patrons peu scrupuleux. Pourtant, et c'est là que pèse l'expérience historique, dans les projets de réforme, il n'est jamais question de renoncer à l'apprentissage sur le lieu de production, pour ne pas se couper de la pratique.

Sur ce point, la conception des réformateurs est cependant fondamentalement contradictoire, car on voit mal comment concilier un système qui demeurerait fondé sur un contrat de droit privé entre l'apprenti et l'entreprise, donc sur une prestation volontaire de l'économie, avec une scolarisation croissante de la formation, c'est-à-dire une augmentation de l'emprise de l'Etat sur celle-ci. C'est bien pourquoi le projet de loi correspondant, malgré quelques concessions faites aux milieux économiques, échoue en 1976 à la deuxième chambre (Bundesrat), où les chrétiens-démocrates ont la majorité.

Malgré cet échec, le gouvernement fait une deuxième tentative de réforme. En effet, au premier débat sur la nature de la formation professionnelle s'en est bientôt ajouté un autre concernant le nombre de places d'apprentissage disponibles. Pour remédier à 
l'insuffisance de l'offre de places d'apprentissage, qui prend des proportions alarmantes avec la crise économique, divers modes de financement public ont été discutés depuis 1973. C'est la formule (modeste), déjà insérée dans la loi de 1976 précédemment évoquée, que le gouvernement cherche à sauver en faisant adopter par le Parlement un texte spécifique (Ausbildungsplatzförderungsgesetz) dès l'automne de la même année : aux termes de ce texte, les entreprises restent maîtresses du financement de leur programme de formation, un financement additionnel de l'Etat n'intervenant que lorsque l'offre globale de places n'excède pas la demande de plus de $12,5 \%^{4}$. Dans ce cas, les subventions accordées proportionnellement à l'effort de formation redistribuent le produit d'une taxe prélevée sur l'ensemble de l'économie, qui ne peut toutefois dépasser $0,25 \%$ de la masse salariale de chaque entreprise et dont sont exonérées les plus petites d'entre elles.

Mais le gouvernement n'a pas plus de succès avec cette loi qu'avec la précédente. Devant les résistances des milieux industriels, il hésite tout d'abord à l'appliquer. Puis, avant même de s'y être résolu, il voit le texte invalidé par le Tribunal constitutionnel en décembre 1980. Il faut toutefois souligner que l'inconstitutionnalité porte sur la procédure d'adoption de la loi, contestable eu égard au mécanisme administratif de financement prévu : bien qu'empiétant sur le domaine de compétence des Länder, le texte n'avait pas été soumis au Bundesrat ${ }^{5}$. Ce n'est donc pas le principe même d'une incitation financière à la création de places d'apprentissage qui a été condamné, et il n'est, par conséquent, pas exclu qu'un gouvernement songe de nouveau à y recourir.

Entre-temps, les débats des années 70 paraissent bien oubliés. Non seulement la majorité conservatrice installée au pouvoir depuis 1982 ne souhaite évidemment pas remettre en question la gestion autonome de l'apprentissage par l'économie, mais le SPD lui-même a fait sa révolution libérale. L'école globale a perdu de son attrait, et les sociaux-démocrates et les syndicats ont redécouvert les vertus d'un modèle d'apprentissage centré sur l'entreprise.

Par ailleurs, la légitimité du système mis en place par la loi de 1969 a été doublement renforcée par l'exercice de la cogestion: d'une part, celle qui a été introduite dans le mécanisme d'élaboration des référentiels d'apprentissage par la loi elle-même et qui, comme nous le verrons, a permis la refonte d'un grand nombre de profils professionnels, à la satisfaction conjointe du patronat et des syndicats; d'autre part, la cogestion du conseil d'entreprise (Betriebsrat) qui, à la faveur des modifications législatives de 1972 (modification du Betriebsverfassungsgesetz), a été étendue à l'apprentissage.

24 Quant au déficit de l'offre, sur lequel nous reviendrons, il a été important dans les années qui ont suivi le deuxième choc pétrolier, bien que l'économie ait fait de gros efforts pour augmenter les capacités de formation. C'est d'ailleurs cette conjoncture qui, avant que les reclassements idéologiques sur l'école se soient opérés, a, au même titre que le changement de majorité, donné un coup d'arrêt au débat sur la réforme. Mais depuis, le déficit est plus que résorbé, au point même que - comme nous le montrons en conclusion - c'est l'insuffisance de candidats à l'apprentissage et par conséquent la menace d'un manque de main-d'œuvre qualifiée qui se profile maintenant dans un certain nombre de secteurs. 


\section{NOTES}

1. Sur tous ces aspects, cf. entre autres Marhild von Behr, Die Entstehung der industriellen Lehrwerkstatt, Francfort s. M., 1981; Roland Ebert, Zur Entstehung der Kategorie Facharbeiter als Problem der Erziehungswissenschaft, Bielefeld, 1984; Karl-Jürgen Rinneberg, Das betriebliche Ausbildungssystem in der Zeit der industriellen Umgestaltung Deutschlands, Cologne/Vienne, 1985 ; Karlwilhelm Stratmann/Manfred Schlösser, Das duale System der Berufsbildung, eine historische Analyse seiner Reformdebatten, Francfort s. M., 1990; WolfDietrich Greinert, Sozialgeschichte der Berufserziehung, die historische Entwicklung des dualen Systems der Berufsausbildung in Deutschland, à paraître.

2. Tel est l'argument principal qu'on trouve par exemple dans la "Déclaration commune de l'économie allemande" de 1962 reproduite dans Gunter Pätzold (dir.), Quellen und Dokumente zur Geschichte des Berufsbildungsgesetzes 1875-1981, Vienne, 1982.

3. Cf. Georg Picht, Die deutsche Bildungskatastrophe, Fribourg, 1964 ; Ralf Dahrendorf, Bildung ist Biirgerrecht, Plädoyer für eine aktive Bildungspolitik, Hambourg, 1965.

4. On estime que, sans un tel excédent calculé globalement, les candidats-apprentis risquent de ne pas disposer dans chaque branche d'un choix suffisant.

5. Sur ces événements, cf. Stratmann/Schlösser, op. cit., et Patzold, op. cit. 


\section{L'apprentissage dual : élément central d'un système diversifié de formation professionnelle}

1 Avant d'analyser sa logique interne et ses résultats, il faut non seulement mettre l'apprentissage dual en perspective historique, mais aussi le situer dans son environnement. Tout comme il réalise en son sein une synthèse entre des traditions et des logiques divergentes, l'apprentissage dual se situe lui-même dans un système de formation professionnelle initiale diversifié. Il y occupe certes une position dominante, mais cohabite aussi avec des types de formation purement scolaires, coexistence qui contribue à la flexibilité globale du système.

\section{La prépondérance du système dual}

2 Quelques chiffres suffisent à illustrer l'importance considérable du duales System. Au 31 décembre 1990, il drainait au total 1476000 jeunes (dont plus de 545000 entrés en apprentissage dans le courant de l'année) ${ }^{1}$. Plus globalement, on peut dire qu'environ $70 \%$ des jeunes passent actuellement par le système dual pour peu que l'on compte tous les apprentis, autant ceux ayant choisi cette voie dès la fin de leur formation générale, que ceux s'y étant engagés après avoir suivi une formation professionnelle scolaire à plein temps, ou dans tout autre cas de figure ${ }^{2}$. 
Figure 1. Nombre total d'apprentis dans les Länder occidentaux 1975-1990

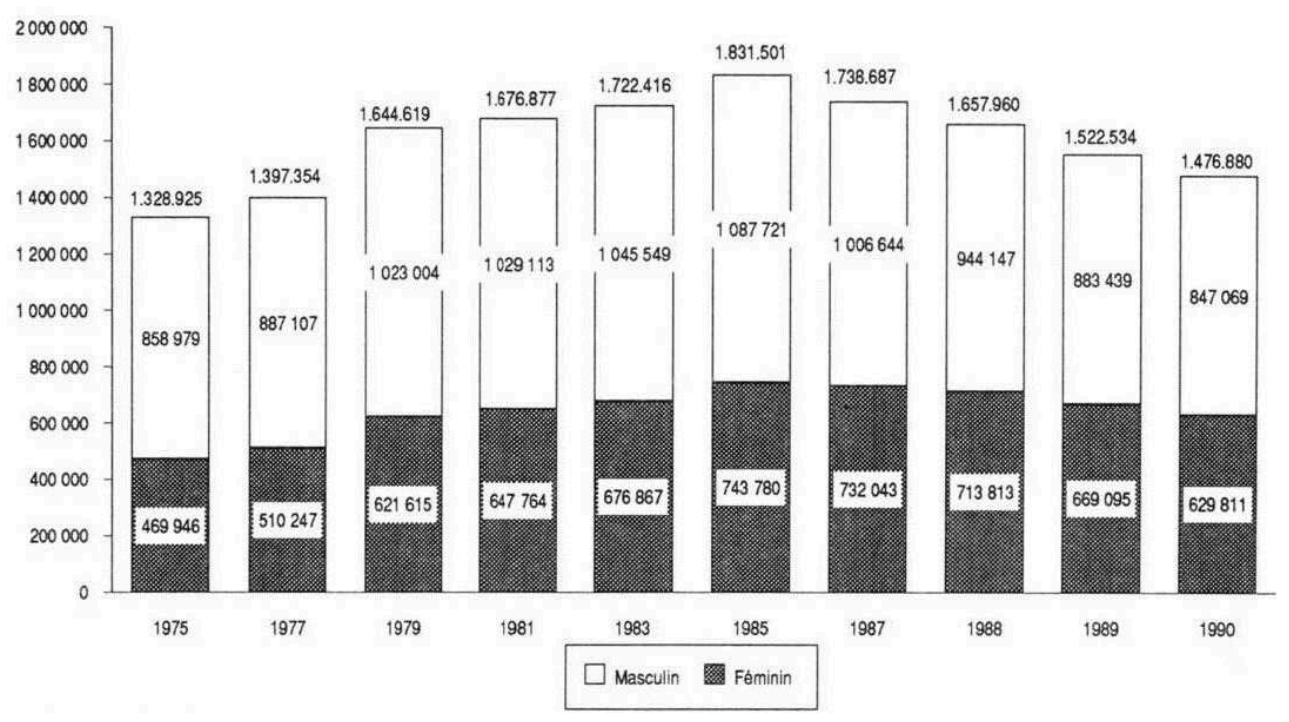

D’après : Berufsbildungsbericht 1992

3 De tels ordres de grandeur seraient évidemment impensables si, dans son acception allemande, le terme d'apprentissage n'avait pas une signification bien plus large qu'en France. La majeure partie des places d'apprentissage dual occupées se trouvent dans l'industrie et le commerce (de 46 à $52 \%$ au cours des deux dernières décennies, 51,2\% en 1990), ainsi que dans l'artisanat (de 34 à $40 \%$ selon les années de la même période, $33,0 \%$ en 1990 - cf. tableau 1).

Tableau 1. Répartition des apprentis du système dual par secteur d'activité dans l'ancienne RFA 1973-1990

\begin{tabular}{|c|c|c|c|c|c|c|c|c|}
\hline \multirow[t]{4}{*}{ Année } & \multicolumn{8}{|l|}{ Apprentis } \\
\hline & \multirow[t]{2}{*}{ Total } & \multicolumn{7}{|l|}{ dont } \\
\hline & & $\begin{array}{c}\text { Industrie } \\
\text { of } \\
\text { commerce }\end{array}$ & Artisanat & $\begin{array}{l}\text { Agri- } \\
\text { culture }\end{array}$ & $\begin{array}{l}\text { Services } \\
\text { publics }\end{array}$ & $\begin{array}{c}\text { Profes- } \\
\text { sions } \\
\text { libérales }\end{array}$ & $\begin{array}{l}\text { Economie } \\
\text { domestique }\end{array}$ & Marine \\
\hline & en milliers & $\%$ & $\%$ & $\%$ & $\%$ & $\%$ & $\%$ & $\%$ \\
\hline 1973 & 1330,8 & 52,5 & 34,9 & 1,9 & 3,7 & 6,7 & 0,5 & 0,1 \\
\hline 1975 & 1328,9 & 47,7 & 38,0 & 2,5 & 3,5 & 7,8 & 0,6 & 0,1 \\
\hline 1977 & 1397,4 & 46,1 & 39,8 & 2,9 & 3,2 & 7,4 & 0,5 & 0,1 \\
\hline 1979 & 1644,6 & 45,5 & 41,1 & 2,8 & 3,3 & 6,7 & 0,5 & 0,1 \\
\hline 1981 & 1676,9 & 46,0 & 40,2 & 2,8 & 3,2 & 7,4 & 0,4 & 0,1 \\
\hline 1983 & 1722,4 & 46,0 & 39,2 & 3,0 & 3,7 & 7,6 & 0,5 & 0,1 \\
\hline 1985 & 1831,5 & 47,8 & 37,5 & 2,9 & 4,0 & 7,2 & 0,6 & 0,1 \\
\hline 1987 & 1738,7 & 49,8 & 35,5 & 2,6 & 4,1 & 7,2 & 0,6 & 0,1 \\
\hline 1988 & 1658,0 & 49,9 & 34,9 & 2,3 & 4,1 & 8,1 & 0,7 & 0,1 \\
\hline 1989 & 1552,5 & 50,4 & 34,3 & 2,2 & 4,0 & 8,3 & 0,7 & 0,1 \\
\hline 1990 & 1476,9 & 51,2 & 33,0 & 2,0 & 4,3 & 8,8 & 0,7 & 0,1 \\
\hline
\end{tabular}

D'APRÈS : BERUFSBILDUNgSBERICHT 1992

4 Cependant, les 374 professions reconnues ${ }^{3}$ du système dual se répartissent dans tous les secteurs de l'économie, donc également dans celui des professions libérales, des 
services publics, de l'agriculture, de l'économie domestique (Hauswirtschaft) et de la marine. A observer par ailleurs la dispersion des professions choisies, on s'aperçoit en outre qu'il existe une tendance à la diversification des choix ${ }^{4}$ (cf. tableau 2). Enfin, ce sont au total 513000 établissements (secteur des services et professions libérales comprises) qui forment dans le cadre du système dual, c'est-à-dire à peu près 1 établissement sur 5, dont le taux d'apprentissage moyen (Ausbildungsquote - part des apprentis dans le total des salariés employés) s'établit à $6,5 \%$.

5 Cette prépondérance du système dual apparaît tendanciellement stable : ainsi, de 1960 à 1980, le poids des apprentis dans la population résidente âgée de 15 à 19 ans se situe constamment entre 30 et $90 \%$. Une telle constance des flux relatifs se traduit évidemment en termes de stock : il n'est donc pas étonnant de constater que pour la même année 1989, la part des diplômés du système dual dans le total des actifs occupés allemands s'établit à plus de $50 \%$.

6 La stabilité globale (en part relative) des entrées dans le système dual recouvre certes des évolutions complexes sur lesquelles nous reviendrons ultérieurement. L'origine des populations qu'il recrute évolue en particulier sous l'effet d'influences structurelles. Mais lorsqu'on observe, comme nous le verrons pour les dernières années (cf. chapitre $\mathrm{VI})$, une érosion résultant du déclin démographique ou des modifications dans les parcours de formation, il faut noter que le système dual perd du terrain par rapport aux formations longues non-professionnelles. Ce n'est donc pas sa suprématie parmi l'ensemble des formations professionnelles qui est remise en cause.

7 Le système dual a également été affecté par des fluctuations conjoncturelles. On ne saurait s'en étonner, car, compte tenu de la place centrale de l'entreprise, soulignée dans le chapitre précédent, il relève fondamentalement d'un modèle de marché : l'offre de formation résulte de la libre décision de l'employeur, liberté défendue jalousement, comme l'ont montré les débats sur l'opportunité d'un financement public additionnel dans les années 70 .

Tableau 2. Les dix professions les plus choisies en 1990 par les apprentis masculins et féminins dans l'ancienne RFA

\begin{tabular}{|c|c|c|c|c|c|c|c|}
\hline \multirow[t]{2}{*}{ Professions } & \multirow[t]{2}{*}{$\begin{array}{l}\text { Secteur } \\
\text { d'apprentissage }\end{array}$} & \multirow[t]{2}{*}{$\begin{array}{l}\text { Apprentis } \\
\text { masculins }\end{array}$} & \multirow[t]{2}{*}{$\begin{array}{l}\begin{array}{l}\text { Part dans le } \\
\text { total des } \\
\text { apprentis } \\
\text { masculins }\end{array} \\
\%\end{array}$} & \multirow[t]{2}{*}{ Protessions } & \multirow[t]{2}{*}{$\begin{array}{l}\text { Secteur } \\
\text { d"apprentissage }\end{array}$} & \multirow[t]{2}{*}{$\begin{array}{l}\text { Apprentis } \\
\text { téminins }\end{array}$} & \multirow{2}{*}{$\begin{array}{l}\text { Part dans le } \\
\text { total des } \\
\text { apprentis } \\
\text { teminins } \\
\%\end{array}$} \\
\hline & & & & & & & \\
\hline Mécanicien automobile & Artisanat & 63297 & 7,5 & Coiffeuse & Artisanat & 46171 & 7,3 \\
\hline Electricien & Artisanat & 44480 & 5,3 & $\begin{array}{l}\text { Agent commercial du } \\
\text { commerce de détail }\end{array}$ & Ind. et commerce & 44328 & 7,0 \\
\hline $\begin{array}{l}\text { Agent commercial du } \\
\text { commerce de gros et du } \\
\text { commerce international }\end{array}$ & Ind. et commerce & 27801 & 3,3 & $\begin{array}{l}\text { Agent commercial de } \\
\text { bureau }\end{array}$ & Ind. et commerce & 42316 & 6,7 \\
\hline $\begin{array}{l}\text { Mecanicien industriel en } \\
\text { technique de production }\end{array}$ & Ind. et commerce & 27738 & 3,3 & Assistante médicale & Prot. liberales & 41925 & 6,7 \\
\hline $\begin{array}{l}\text { Mécanicien industriel en } \\
\text { technique des machines } \\
\text { et des systèmes }\end{array}$ & Ind. et commerce & 27715 & 3,3 & $\begin{array}{l}\text { Agent commercial } \\
\text { d'industrie }\end{array}$ & Ind. et commerce & 39157 & 6,2 \\
\hline Menuisier & Artisanat & 27267 & 3,2 & Assistante dentaire & Prot. liberales & 30666 & 4,9 \\
\hline Agent commercial bancaire & Ind. ef commerce & 25732 & 3,0 & $\begin{array}{l}\text { Vendeuse qualifiée dans } \\
\text { lantisanat alimentaire }\end{array}$ & Artisanat & 30187 & 4,8 \\
\hline Agent commercial dindustrie & $\theta$ ind. et commerce & 24059 & 2,8 & $\begin{array}{l}\text { Agent commercial } \\
\text { bancaire }\end{array}$ & Ind. et commerce & 29501 & 4,7 \\
\hline $\begin{array}{l}\text { Agent commercial du } \\
\text { commerce de détail }\end{array}$ & Ind. et commerce & 23905 & 2,8 & $\begin{array}{l}\text { Agent commercial du } \\
\text { commerce de gros et du } \\
\text { commerce international }\end{array}$ & Ind. et commerce & 20616 & 3,3 \\
\hline Installateur gaz et plomberie & Artisanat & 22730 & 2,7 & Vendeuse & Ind. et commerce & 19992 & 3,2 \\
\hline Total & & 314724 & 37,2 & Total & & 344859 & 54,8 \\
\hline
\end{tabular}

D’après : Berufsbildungsbericht 1992 
Au niveau macro-économique, le principal risque d'un modèle marchand est donc l'insuffisance de l'offre d'apprentissage. La RFA a connu cette situation pendant quelques années après le premier choc pétrolier, puis plus longuement et plus gravement de 1982 à 1986/87, lorsqu'à la profonde récession s'ajoutait le poids des classes d'âge nombreuses sortant du système scolaire. Dans cette situation difficile, le système dual a cependant tiré parti du caractère mixte de sa régulation. Entre les modèles purement marchands (Japon et Etats-Unis) et purement scolaires (France), il occupe en effet une position médiane ${ }^{7}$. La logique de marché y est tempérée par une régulation d'Etat, qu'il s'agisse de régler la coopération avec le système scolaire ou de sanctionner les compromis d'une négociation institutionnalisée avec les syndicats. Certes, les pouvoirs publics ne peuvent forcer les entreprises à recruter des apprentis, mais ils disposent d'une marge de manœuvre non négligeable pour épauler le marché défaillant.

9 Dans la période en question, ils ont pris toute une série d'initiatives en ce sens. Dans le cadre de la politique de l'emploi, l'office fédéral du travail (Bundesansta.lt für Arbeit) a notamment renforcé les mesures profitant aux jeunes ${ }^{8}$ tandis que le gouvernement fédéral mettait en place des aides à l'emploi pour les groupes défavorisés (Benachteiligtenprogramm) - offrant une formation hors entreprise ou un soutien à l'apprentissage ${ }^{9}$ et que les Länder accordaient des subventions aux entreprises et augmentaient les capacités d'accueil des formations professionnelles purement scolaires (Berufsfachschulen, Berufsgrundbildungsjahr, Berufsvorbereitungsjahr) ${ }^{10}$. Par ailleurs, les critiques de plus en plus vives adressées au système dual conduisaient les milieux patronaux à augmenter de façon volontariste l'offre d'apprentissage pour essayer de restaurer la légitimité menacée du système ${ }^{11}$.

\section{Les formations professionnelles scolaires à plein temps}

10 A côté du système d'apprentissage dual existe aussi un secteur de formation professionnelle scolaire à plein temps. Ses caractéristiques, très variables, reflètent, pour une part, des particularités régionales ou des spécificités sectorielles. Le statut des établissements, qui portent le titre générique de Berufsfachschulen, est public, semi public ou privé, avec dans ces deux derniers cas une liaison plus ou moins étroite avec les chambres de commerce ou les chambres des métiers. Les études suivies peuvent donner une qualification professionnelle complète, soit (pour une faible part) dans des métiers couverts par ailleurs par l'offre du système dual ${ }^{12}$ soit pour des professions exclusivement préparées en milieu scolaire (comme bon nombre de professions de santé et d'assistants techniques dans l'industrie). Mais elles peuvent aussi ne donner qu'une formation professionnelle de base, avec, dans certains cas, l'acquisition simultanée d'un diplôme de formation générale. Le parcours d'études ultérieur peut alors déboucher sur le système dual ou retourner vers la formation nonprofessionnelle. Selon les cursus, la scolarité dans les Berufsfachschulen dure de 1 à 3 ans.

\section{Les écoles professionnelles en Allemagne}

Les strates de l'histoire et les cloisonnements du fédéralisme expliquent la grande différenciation des écoles professionnelles, dont nous ne pouvons rendre 
pleinement compte ici. Cependant, une simple présentation des grands types d'établissements met bien en évidence, malgré l'inévitable schématisme, le rôle structurant du système dual et l'importance des "passerelles". Grâce à ces deux facteurs, la diversité n'est pas synonyme d'éclatement, mais va de pair avec une flexibilité qui permet réorientations et poursuites d'études. Les chiffres indiqués sont ceux de 1990.

A part la Berufsschule, qui occupe une place centrale conforme à la prédominance du système dual, il existe des écoles professionnelles qui remplissent une ou plusieurs des fonctions suivantes : préparer à la formation dans le système dual (Berufsgrundbildungsjahr et certaines Berufsfachschulen), compléter l'éventail de professions offert par celui-ci - plus rarement le concurrencer sur son propre terrain (Berufsfachschulen), acquérir une qualification de technicien (Fachschulen), rattraper un diplôme scolaire de formation générale (Berufsaufbauschule, Fachoberschule et certaines Berufsfachschulen).

Les Berufsschulen (1,4 million d'élèves, dont environ 600000 en première année) sont fréquentées par les apprentis du système dual, mais aussi, en vertu de l'obligation scolaire jusqu'à 18 ans, par les jeunes entrés directement dans la vie professionnelle à la sortie du système d'enseignement général. L'enseignement, d'une durée équivalant à 8-10 heures par semaine, y est dispensé à temps partiel, soit 1 ou 2 jours par semaine, soit par blocs de plusieurs semaines par an (p. ex. 2 fois 6 semaines). Il comprend principalement les matières théoriques correspondant à la spécialité professionnelle choisie (avec éventuellement des exercices dans l'atelier de la Berufsschule), mais aussi de l'allemand, de l'instruction civique, du sport et un enseignement religieux.

La Berufsgrundbildungsjahr, formation professionnelle de base d'un an, n'existe que dans certains Länder et pour certaines professions. En 1990, elle concernait au total 83000 jeunes. Se substituant à la première année d'apprentissage dual, elle initie non pas à une profession, mais à l'ensemble d'un champ professionnel. Elle peut se dérouler entièrement dans le cadre scolaire ou en statut d'alternance (mode “coopératif"), c'est-à-dire avec un contrat d'apprentissage. Dans ce cas, le temps passé à la Berufsschule est de 2 ou même de 2 jours et demi par semaine.

Les Berufsfachschulen (245000 élèves dont 153000 en première année + 90000 élèves dans les écoles du secteur de santé, dont 45 à 50000 en première année) sont des écoles professionnelles à plein temps dont le cursus est d'au moins 1 an. Elles accueillent des jeunes ayant le certificat de fin de Hauptschule et ont trois types de débouchés. Elles conduisent à des diplômes professionnels qu'elles sont seules à décerner ou (plus rarement) dans des spécialités auxquelles forme également le système dual. Dans ces deux cas, la scolarité dure en général 3 ans. Sinon, et c'est plus fréquent, elles donnent une formation professionnelle nondiplômante, mais permettant d'intégrer le système dual en cours de route. La scolarité dure alors 1 ou en général 2 ans, auquel cas est souvent également délivré en fin de cursus un équivalent du certificat de fin d'école moyenne (Fachschulreife, équivalent à la Mittlere Reife), ce qui permet aussi de poursuivre des études dans l'enseignement général.

Les Berufsaufbauschulen sont ouvertes aux élèves sortis de la Hauptschule qui suivent depuis au moins 6 mois une formation professionnelle ou l'ont achevée. Selon les cas, l'enseignement y est respectivement à temps partiel (le soir) et s'étend sur plusieurs années, ou à plein temps et d'une durée d'un an. Les 
Berufsaufbauschulen ont été conçues à la fois pour approfondir la formation professionnelle et acquérir le certificat de fin d'école moyenne, mais c'est cette dernière fonction qui, dans la pratique, l'emporte. Les Berufsaufbauschulen ont perdu de leur importance (8 000 élèves seulement), surtout depuis la transformation à la fin des années 60 des anciennes écoles techniques supérieures (höhere Fachschulen) auxquelles elles permettaient d'accéder, en instituts techniques du supérieur (Fachhochschulen). Appartenant désormais à la sphère universitaire, ces Fachhochschulen requièrent en effet un certificat scolaire spécifique (Fachhochschulreife).

Ce certificat est décerné par la Fachoberschule à laquelle ont accès les titulaires du certificat de fin d'école moyenne (Mittlere Reife) ou d'un équivalent (Fachschulreife) - 80000 élèves environ. La scolarité dure de 1 à 3 ans en fonction du parcours professionnel antérieur et selon qu'elle est suivie à plein temps ou à temps partiel. L'enseignement allie des contenus pratiques et une formation théorique. Les Fachschulen (115 000 élèves) sont des écoles de perfectionnement qui s'adressent aux titulaires d'un diplôme professionnel ayant exercé leur métier un certain nombre d'années et permettent l'accès aux qualifications de technicien. Leurs cursus durent au moins 1 an (à plein temps) et jusqu'à 4 ans (à temps partiel). Ils couvrent un grand nombre de domaines, techniques (notamment pour les qualifications de contremaître - Industriemeister), commerciaux, pédagogiques, de santé, etc. En même temps que le diplôme professionnel (reconnu par l'Etat), elles décernent souvent l'équivalent du certificat de fin d'école moyenne ou la Fachhochschulreife.

Quant aux Fachgymnasien, lycées techniques (environ 20000 élèves), ouverts aux titulaires d'un certificat de fin d'école moyenne (Mittlere Reife), ils peuvent conduire, après 3 ans de scolarité à plein temps, à des qualifications intermédiaires dans la vie professionnelle. Mais beaucoup plus souvent, ils débouchent sur une poursuite des études à la Fachhochschule, dont ils décernent le certificat d'entrée (Fachhochschulreife), ou dans une université technique. 


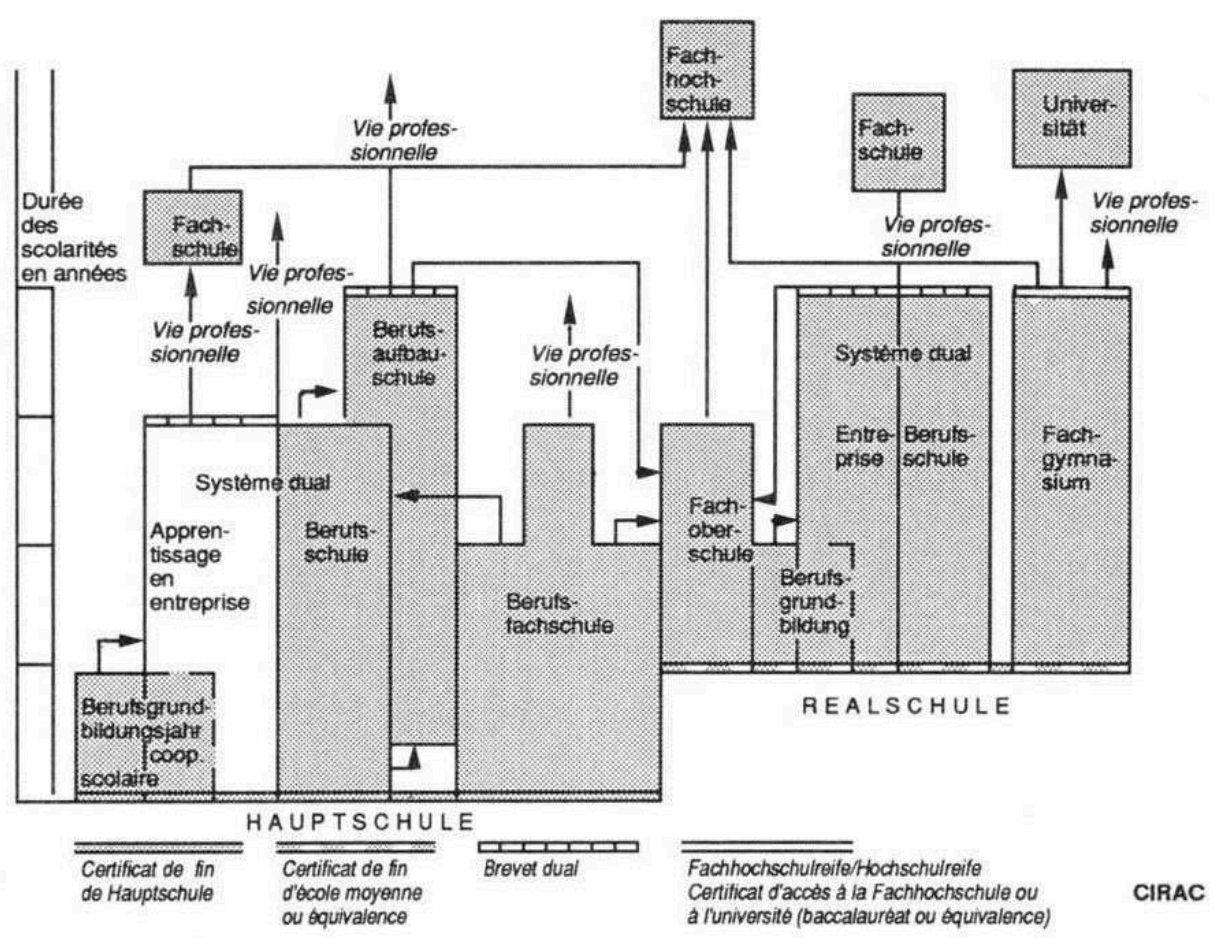

11 En 1990, 245000 jeunes au total étaient en formation dans des Berufsfachschulen techniques ou commerciales (153000 jeunes y commençaient leur scolarité), dont $20 \%$ suivaient des cursus de 2 à 3 ans, donnant une qualification professionnelle complète. S'y ajoutent les 90000 élèves des écoles formant aux professions paramédicales ${ }^{13}$. Si on met ces dernières à part - car elles ont un monopole de formation -, on voit donc que moins de $15 \%$ des jeunes en formation professionnelle initiale échappent au système dual. Drainant 6/7 des effectifs, celui-ci est donc bien la filière dominante ${ }^{14}$.

Certes, on observe que la part des élèves d'écoles professionnelles à plein temps a considérablement augmenté depuis les années 50 : à l'époque, celles-ci n'accueillaient que 7 à $8 \%$ des jeunes en formation professionnelle initiale. L'expansion intervenue depuis s'est faite surtout dans le domaine commercial et a drainé dans une large mesure un public féminin. Mais compte tenu de l'hétérogénéité du secteur des Berufsfachschulen, ces chiffres ne reflètent que partiellement un rapport de concurrence entre les deux modes de formation. Ainsi, dans le Bade-Wurtemberg, pour certains métiers, l'école professionnelle d'un an à plein temps est le préalable obligatoire à la formation duale.

En fait, la modification des parts relatives du secteur dual et du secteur purement scolaire reflète bien plus des évolutions conjoncturelles que des modifications structurelles. En effet, la part relative des Berufsfachschulen se situait déjà au niveau actuel au début des années 70 . Augmentant ensuite continûment pour s'établir aux alentours de $17 \%$ dans la première moitié des années 80 , avant d'amorcer un mouvement de reflux qui lui fait retrouver son niveau initial, elle suit une évolution inverse ment proportionnelle à celle du solde de l'offre et la demande sur le marché des places d'apprentissage dual. Il y a certes l'exception du secteur commercial des écoles professionnelles à plein temps, dont les chiffres d'inscription ne refluent pas, preuve 
qu'elles satisfont un besoin de formation intermédiaire spécifique ; mais globalement, les jeunes se sont donc d'autant plus tournés vers les écoles professionnelles à plein temps que le système dual avait tendance à leur fermer ses portes. Le secteur des Berufsfachschulen a plus joué un rôle d'amortisseur que de concurrent.

La même remarque vaut pour un autre élément de formation professionnelle scolaire, la formation professionnelle de base d'un an (Berufsgrundbildungsjahr - BGJ). Discutée depuis la fin des années 60, celle-ci a été mise en œuvre à partir du début des années 70 . En préconisant la scolarisation de la première année d'apprentissage, certains réformateurs poursuivaient plusieurs objectifs : faciliter la transition entre école et formation, notamment en évitant les orientations prématurées et hasardeuses, assurer une meilleure formation théorique préalable, élargir les bases de qualification, remédier aux différences régionales et sectorielles dans l'offre d'apprentissage, enfin augmenter les capacités d'accueil du système de formation professionnelle.

La réforme a rencontré une forte résistance du patronat qui lui reproche de manière générale de neutraliser les mécanismes du marché, mais aussi plus concrètement de voler inutilement du temps à la formation spécialisée et d'être fondée sur un découpage discutable de 13 "champs professionnels de base" (Berufsfelder). Face à cette opposition, la BGJ n'a pas été instaurée que sous forme scolaire, comme il était initialement prévu, mais existe aussi sous forme dite "coopérative", où, tout en respectant le principe d'une initiation dans des grands champs professionnels, subsiste l'alternance entre l'école (.Berufsschule) - dont l'horaire est renforcé - et l'entreprise. Rapidement au centre des controverses politiques sur le système éducatif, la formation professionnelle de base a été de plus très inégalement appliquée selon les Länder ${ }^{15}$.

Si l'on considère le nombre de jeunes concernés - jamais plus de $20 \%$ du total des apprentis de première année - on voit clairement que la réforme a échoué. Cet échec illustre doublement le caractère dominant du modèle dual : tout d'abord, l'examen des chiffres montre que les flux d'élèves drainés par la BGJ (à peine plus de 100000 en 1982/83, année d'expansion maximale) ${ }^{16}$ sont, comme pour les Berufsfachschulen, inversement proportionnels à la situation sur le marché de l'apprentissage. Après avoir aidé à amortir la pénurie des places d'apprentissage dual, l'influence de la formation professionnelle de base scolaire n'a cessé de décliner : elle ne touche aujourd'hui guère plus de $15 \%$ des jeunes en début de formation. Par ailleurs, l'apprentissage dual n'a pas recouvré son influence que grâce à l'inertie du système ${ }^{17}$; comme nous le verrons plus précisément au chapitre IV, il a pu s'affirmer par sa capacité à intégrer une partie des préoccupations du $\mathrm{BGJ}$ : c'est ainsi que les référentiels professionnels récemment refondus prévoient une première année de formation de base et que les efforts pour construire des centres d'apprentissage inter-entreprises sont aussi une manière de répondre aux déficits régionaux de l'offre.

\section{NOTES}

1. Rappelons que l'apprentissage dure 3 ans ou 3 ans et demi. 
2. Chiffres tirés de : Bundesministerium für Bildung und Wissenschaft, Berufsbildungsbericht 1992, Bad Honnef, 1992 et de : Schlußbericht der Enquête-Kommission "Zukünftige Bildungspolitik - Bildung 2000", berufliche Erstausbildung, Minderheitsvotum, Deutscher Bundestag, Drucksache 11/7820, p. 64. 3. Berufsbildungsbericht 1992, op. cit., p. 91.

4. Précisons que, dans le même temps, conformément aux efforts pour diminuer le nombre des qualifications parcellaires, le nombre de professions reconnues du système dual passait de plus de 500 à 374. Cf. Berufsbildungsbericht 1992, op. cit., p. 91.

5. Cf. Rudolf Werner, "10\% der Auszubildenden in Grossbetrieben", in: Berufsbildung in Wissenschaft und Praxis, 4/1990, p. 35. Les chiffres reposent sur le recensement des lieux de travail (Arbeitsstättenzählung), fait en 1987. Pour des données plus complètes, cf. chapitre IV.

6. "Bildungsbeteiligung stieg weiter", in : DIW Wochenbericht 45/88, 10/11/88. Pour des données plus complètes, cf. chapitre VII.

7. Cf. Wolf-Dietrich Greinerî, "Marktmodell - Schulmodell - duales System, Grundtypen formalisierter Berufsbildung", in : Die berufsbildende Schule 3/1988.

8. II s'agit tout d'abord des mesures dites "de préparation professionnelle" (Berufsvorbereitungsmaßnahmen), prévues initialement pour des jeunes ayant des difficultés scolaires ou un handicap, mais palliant de plus en plus le manque de places d'apprentissage ; parallèlement, l'office fédéral multiplie presque par trois les emplois d'utilité collective (Arbeits beschaffungsmaßnahmen) et les stages de formation et de reconversion. Pour l'année 1986/87, toutes ces mesures concernaient plus de 170000 jeunes. Cf. à ce sujet Frank Strikker, "Veränderungen der Berufsausbildung unter dem Aspekt mangelnder Integrationsfähigkeit des Arbeitsmarktes", in : Frank Strikker/Dieter Timmermann, Berufsausbildung und Arbeitsmarkt in den 90er Jahren, Francfort s. M., 1990.

9. En 1986/87, plus de 35000 jeunes ont profité de ces programmes.

10. L'effet des subventions destinées à susciter la création de places d'apprentissage n'a, à notre connaissance, pas été chiffré. Pour toutes les autres mesures, cf. Strikker, op. cit.

11. Le risque de remise en cause du système dual était définitivement écarté depuis le changement de majorité parlementaire en 1982. Nous avons observé cet effort de formation "au delà des besoins" en analysant les chiffres de toutes les grandes entreprises que nous avons visitées (Bayer, Volkswagen, Bosch, Gerling, DKV). Cet effort spontané, sorte de "réaction immunitaire" du système, ainsi que les mesures de l'Etat ont permis d'amortir le choc de la crise. Il est clair cependant que le déficit de places d'apprentissage n'a pas été complètement comblé. De plus, les mesures d'accompagnement ont dans bien des cas permis aux jeunes d'attendre, mais ne leur ont pas forcément ouvert l'accès à une formation professionnelle. Les estimations les plus pessimistes font état de presque 150000 jeunes par an qui n'auraient pas pu trouver de formation professionnelle pendant les 10 années de crise. Le chiffre est cité dans : Deutscher Bundestag, Schlußbericht der Enquête-Kommission "Zukünftige Bildungspolitik - Bildung 2000", Bonn, Drucksache 11/7820, 05/09/1990, Minderheitsauffassung, p. 66. Cf. aussi les estimations comparées dans: Karlwilhelm Stratmann/Manfred Schlösser, Das duale System der Berufsbildung. Eine historische Analyse seiner Reformdebatten, Francfort s. M., 1990, p. 252.

12. Citons, à titre d'exemple, les écoles de "l'élite ouvrière", préparant aux artisanats d'art.

13. Berufsbildungsbericht 1992, op. cit., p. 43 sq.

14. Pour les chiffres concernant les parts relatives des apprentis du système dual et des élèves de Berufsfachschulen, les calculs ont été faits à partir de Stratmann/Schlösser, Das duale System der Berufsbildung, op. cit.

15. Il n'y a pas un seul champ professionnel où la BGJ soit mise en place dans l'ensemble des Länder.

16. Cf. Wolf-Dietrich Greinert, Das duale System der Berufsbildung in der BRD, Struktur und Funktion, Eschborn, 1992. 
17. ... dont fait partie aussi la résistance patronale à reconnaître la BGJ équivalente à la première année de formation duale, malgré les textes réglementaires qui le stipulent formellement. 


\section{L'entreprise formatrice au centre d'un système de régulation concertée}

1 Le système dual s'intègre dans un dispositif institutionnel complexe qui assure une régulation-conciliation entre les logiques et les intérêts contradictoires des trois grands partenaires concernés: l'Etat, le patronat et les syndicats, mais qui préserve l'autonomie et le rôle prééminent de l'entreprise dans la mise en œuvre de la formation professionnelle initiale.

2 Au niveau global, le dispositif met en jeu toute une série de procédures dont l'objectif consiste à trouver des arbitrages satisfaisants, et surtout des compromis opérationnels, entre trois grands types d'enjeux :

- régulation centrale et autonomie régionale : il s'agit de concilier, d'une part, le principe constitutionnel du fédéralisme scolaire, qui implique la souveraineté quasi-totale des Länder en matière de politique éducative et scolaire, et d'autre part, la nécessité économique d'un contrôle central de la formation professionnelle en entreprise, garanti par une compétence fédérale exclusive. C'est en effet à l'Etat fédéral (Bund) que la Loi fondamentale confie la responsabilité économiquement vitale d'assurer l'homogénéité du marché du travail et la qualité des qualifications au sein des professions sur l'ensemble du territoire national ;

- action de l'Etat et subsidiarité : il s'agit de concilier le principe d'une action codificatrice et d'un nécessaire contrôle de l'Etat en matière éducative avec celui, tout aussi crucial dans la culture économique et sociale allemande, de la subsidiarité. Ce principe reconnaît et consacre l'autonomie des agents économiques et des partenaires sociaux ainsi que leur droit fondamental à s'auto-organiser et à gérer eux-mêmes les questions qui les concernent directement (Selbstverwaltung) dans leur sphère immédiate d'activité, en l'occurrence la définition et la transmission des qualifications requises dans la vie professionnelle au sein de l'entreprise; l'entreprise étant selon ce même principe mieux habilitée que toute autre instance à développer les qualifications dont elle a besoin ;

- besoins de l'entreprise et droits des salariés: il s'agit enfin de concilier les intérêts économiques et sociaux différents, parfois antagoniques, des facteurs de production indissociables que sont le capital et le travail. Cela implique la concertation nécessaire des organisations patronales et des organisations syndicales de salariés, dans la gestion des 
qualifications. A cet égard, l'enjeu est fondamental pour la gestion des ressources humaines et pour la vie économique, car de la définition des qualifications dépend non seulement l'organisation du travail dans les entreprises, mais aussi l'échelle des classifications et des rémunérations.

\section{L'Institut fédéral pour la formation professionnelle (Bundesinstitut fiir Berufsbildung - BIBB)}

L'Institut fédéral de formation professionnelle (Bundesinstitut fur Berufsbildung) a succédé à la commission fédérale de formation professionnelle créée par la loi sur la formation professionnelle du 14 juin 1969. Son statut actuel a été défini par la loi de promotion de la formation professionnelle (Berufsbildungsförderungsgesetz) du 23 décembre 1981. Etablissement public dépendant directement des autorités fédérales et placé sous la tutelle du ministère fédéral de la Formation et de la Science, l'Institut est installé à la fois à Bonn et à Berlin, la plus grande partie de son potentiel (plus de 300 collaborateurs sur un total de 380 ) travaillant à Berlin. Financé pour l'essentiel par le gouvernement fédéral, il dispose d'un budget d'environ 40 millions de DM, dont plus de 30 millions sont toutefois absorbés par les frais de personnel.

Sa fonction est double : d'une part, il remplit une mission de recherche et de développement en matière de formation professionnelle. Dans ce domaine, s'il n'est pas seul, puisqu'il existe une trentaine d'instituts universitaires de pédagogie professionnelle (Wirtschafts-und Berufspädagogik), où sont par ailleurs formés les professeurs d'école professionnelle, il représente la plus forte concentration de moyens et de compétences ; d'autre part, il agit en tant que conseiller et prestataire de services auprès du gouvernement fédéral et de tous les acteurs de la formation professionnelle. La composition de son principal organe dirigeant, la commission centrale (Hauptausschu $\beta$ ) où sont représentés à poids égal (11 voix) les organisations d'employeurs, les syndicats, les Länder et le gouvernement fédéral reflète bien ce rôle fédérateur. Il faut ajouter un représentant des communes et de l'Office fédéral du travail, qui ne participent toutefois pas aux votes. La commission centrale adopte le programme de recherche et fixe le budget ; elle s'exprime sur l'organisation du système de formation professionnelle, adopte les projets de règlements professionnels élaborés dans le cadre de l'Institut et vote une résolution concernant le rapport sur l'enseignement professionnel présenté chaque année par le ministre fédéral de la Formation et de la Science.

Les principaux domaines de travail de l'Institut sont la recherche sur les parcours professionnels et de formation ainsi que sur les processus d'apprentissage, l'élaboration des règlements de formation, la gestion de projets-pilotes financés par le gouvernement fédéral, la promotion des centres d'apprentissage interentreprises (financée hors-budget par l'Etat fédéral), la conception de programmes de formation continue pour les formateurs, le contrôle et l'agrément de programmes de télé-enseignement, ainsi que l'établissement de statistiques sur la formation professionnelle. 


\section{Un système de régulation tripartite à plusieurs niveaux}

3 Il découle de cette régulation triangulaire un système à plusieurs étages où l'on distingue différents niveaux de compétences (fédéral, sectoriel, régional, entreprise) à l'intérieur desquels interviennent et coopèrent sous des formes et à des fins diverses (réglementation, financement, gestion/mise en œuvre, contrôle) de multiples acteurs (pouvoirs publics fédéraux et régionaux, représentants de l'enseignement public, patronat, organismes consulaires et employeurs, syndicats et conseils d'entreprises). Le système apparaît quelque peu opaque à l'observateur français qui est habitué à la primauté du pilotage centralisé de l'Etat subsidiairement tempéré par la consultation des partenaires sociaux et à une gestion très partiellement déconcentrée au niveau régional et local.

4 Pour l'essentiel, on distingue dans l'organisation du système allemand de formation professionnelle quatre niveaux significatifs de compétence et de régulation (cf. tableau synoptique ci-après) :

\section{Le niveau fédéral interprofessionnel}

5 Le ministère fédéral de la Formation et de la Science (Bundesministerium für Bildung und Wissenschaft - BMBW) exerce une fonction de législation, de contrôle et de coordination globale. Le cadre législatif de référence est la loi fédérale pour la formation professionnelle adoptée en 1969 (voir encadré au chapitre I). Celle-ci fournit un cadre général qui définit les principes de base auxquels doit se conformer toute entreprise souhaitant développer une activité de formation. En l'occurrence, il n'y a pas en Allemagne d'obligation légale de formation pour l'entreprise, mais au contraire pour celle-ci des conditions à satisfaire pour être habilitée à former des apprentis (elle doit en assurer les frais, disposer d'équipements techniques suffisants et de formateurs qualifiés et agréés).

6 L'Etat fédéral met en outre à la disposition des professions un potentiel important d'expertise en matière d'étude des qualifications et des professions par l'intermédiaire du Bundesinstitut für Berufsbildung (BIBB - Institut fédéral pour la formation professionnelle), dans lequel les partenaires sociaux sont représentés à côté des pouvoirs publics (Bund et Länder) au sein d'une ommission centrale tripartite. Les travaux du BIBB servent de base à l'adoption des règlements de formation dans les différentes professions.

7 Pour la partie scolaire de la formation, qui incombe aux Länder, existent des programmes-cadres pour les Berufsschulen qui sont élaborés au plan national par la conférence des ministres de l'Education des Länder (Kultusministerkonferenz - KMK)

\section{Le niveau fédéral de la branche professionnelle}

8 C'est à ce niveau que sont principalement définis les règlements de formation pour les différents métiers de la branche industrielle ou artisanale considérée (Ausbildungsordnungen). Ces règlements de formation sont élaborés de manière concertée au sein de commissions pluripartites dans lesquelles siègent, à égalité de droits, des représentants de l'administration fédérale, des experts compétents du BIBB, des représentants du patronat et des chambres consulaires, des représentants des 
organisations syndicales, les uns et les autres étant compétents ou représentatifs dans le secteur professionnel considéré. Ces réglementations élaborées en commun par ces commissions sont ensuite promulguées sous forme de décrets par le ministère fédéral correspondant au secteur d'activité concerné et s'imposent à la profession, ce qui implique que toute entreprise qui forme des apprentis doit impérativement s'y conformer dans l'organisation et la mise en œuvre de ses plans de formation.

\section{Le niveau régional interprofessionnel et professionnel}

9 A ce niveau est exercé le contrôle de l'application des règlements de formation sur le terrain, tant par les entreprises que par les écoles professionnelles. L'habilitation et le contrôle des entreprises incombe aux chambres d'industrie ou d'artisanat (selon l'affiliation), la tutelle sur les écoles étant exercée par le ministère de l'Education du Land. La certification des diplômes professionnels est cependant assurée par les chambres consulaires, qui ont la responsabilité d'organiser les examens (en coopération avec les formateurs d'entreprise et les enseignants de l'école professionnelle) et de contrôler l'acquisition des compétences.

10 C'est également au niveau régional qu'est organisée la nécessaire coopération entre les représentants $\mathrm{du}$ monde professionnel (employeurs, organismes consulaires et syndicats) et les représentants du système éducatif du Land (niveau de compétence exclusive en matière scolaire) pour la coordination des programmes et l'organisation de l'alternance entreprise-école dans les différents secteurs de l'économie régionale.

\section{Le niveau de l'entreprise}

11 L'entreprise assure, sur la base du contrat individuel de formation, la responsabilité entière de la formation en assumant, dans le strict respect du cadre réglementaire existant, toutes les obligations matérielles (financement, équipement, rémunération des apprentis et des formateurs), sociales (accueil et tutorat) et morales (qualité de la formation et succès final de l'apprenti) qui en découlent. L'entreprise ne peut cependant s'acquitter convenablement de cette mission qu'en coopération étroite avec d'autres partenaires :

- la représentation salariée de l'entreprise, le conseil d'entreprise ayant légalement un droit de codécision sur la politique de formation (loi sur l'organisation interne des entreprises Betriebsverfassungsgesetz);

- l'école professionnelle, pour le bon déroulement du plan de formation et le suivi pédagogique des apprentis ;

- ou bien encore d'autres entreprises dans le cadre des centres de formation inter-entreprises lorsque l'entreprise formatrice est une PME ne disposant pas de moyens techniques suffisants pour assurer seule sa formation.

La répartition des compétences dans le système dual

\begin{tabular}{|l|l|l|}
\hline & $\begin{array}{l}\text { Fixation des objectifs et des } \\
\text { contenus de formation }\end{array}$ & Financement \\
\hline
\end{tabular}




\begin{tabular}{|c|c|c|}
\hline Niveau fédéral & 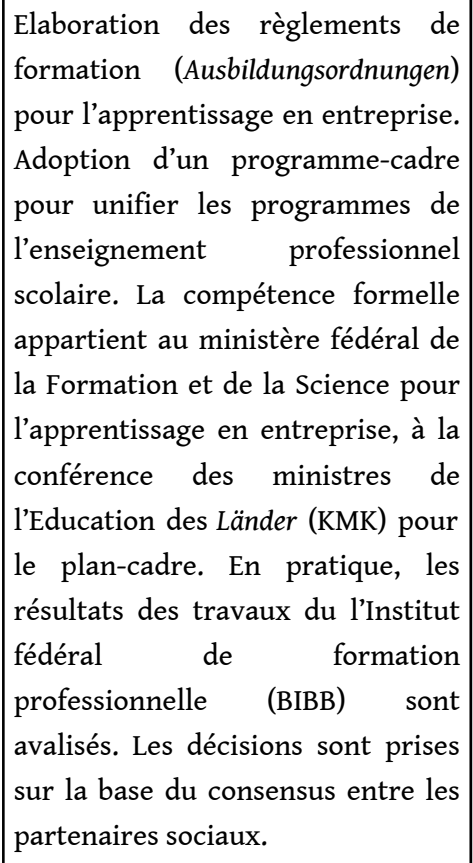 & $\begin{array}{l}\text { Normalement, pas de } \\
\text { ressources mobilisées à } \\
\text { ce niveau. En fait, l'Etat } \\
\text { fédéral peut intervenir } \\
\text { en subventionnant l'offre } \\
\text { de places } \\
\text { d'apprentissage, } \\
\text { notamment pour les } \\
\text { catégories de jeunes en } \\
\text { difficulté (comme il l'a } \\
\text { fait dans les années de } \\
\text { pénurie). En outre, les } \\
\text { organisations de } \\
\text { représentatives d'économie peuvent } \\
\text { l'écon de } \\
\text { exhorter les entreprises à } \\
\text { offrir davantage de } \\
\text { places d'apprentissage } \\
\text { (cf. chap. II). }\end{array}$ \\
\hline Niveau sectoriel & \begin{tabular}{|l} 
Rôle primordial des représentants \\
des partenaires sociaux à ce niveau \\
dans l'élaboration des règlements \\
de formation. Ils sont délégués au \\
niveau fédéral ou consultés lors de \\
l'élaboration.
\end{tabular} & $\begin{array}{l}\text { Normalement, pas de } \\
\text { ressources mobilisées à } \\
\text { ce niveau, mais il existe } \\
\text { dans certaines branches } \\
\text { des fonds créés par } \\
\text { conventions collectives } \\
\text { (p. ex. le bâtiment, cf. } \\
\text { chap. VI) auxquels } \\
\text { versent les entreprises } \\
\text { qui ne forment pas elles- } \\
\text { mêmes, afin } \\
\text { cofinancer la formation } \\
\text { des autres entreprises ou } \\
\text { de financer des centres } \\
\text { de formation } \\
\text { interentreprises. } \\
\text { Négociation à ce niveau } \\
\text { des rémunérations des } \\
\text { apprentis, qui rejaillit sur } \\
\text { le coût de la formation. }\end{array}$ \\
\hline
\end{tabular}




\begin{tabular}{|c|c|c|}
\hline Niveau régional & 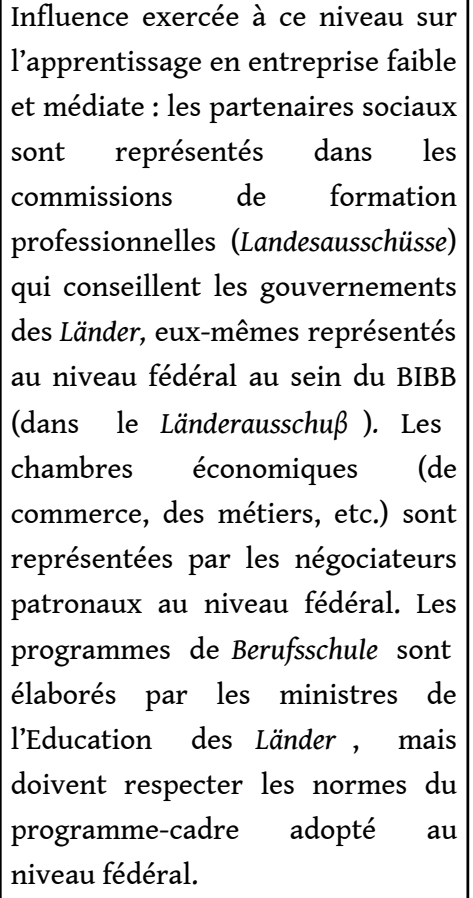 & $\begin{array}{l}\text { Financement de la partie } \\
\text { scolaire }\end{array}$ \\
\hline Niveau des entreprises & $\begin{array}{l}\text { Niveau de compétence effectif, } \\
\text { dans la mesure où les entreprises } \\
\text { peuvent donner une formation de } \\
\text { niveau supérieur aux normes du } \\
\text { règlement d'apprentissage. C'est } \\
\text { surtout le cas dans les grandes } \\
\text { entreprises. } \\
\text { d'entreprise, qui ont un droit de } \\
\text { codécision sur la manière dont est } \\
\text { effectuée la formation, y exercent } \\
\text { leur influence en ce sens. }\end{array}$ & $\begin{array}{l}\text { Financement de la } \\
\text { formation en entreprise } \\
\text { par les entreprises elles- } \\
\text { mêmes. }\end{array}$ \\
\hline \multirow[t]{2}{*}{ Mise en œuvre et gestion } & Evaluation et contrôle & \\
\hline & $\begin{array}{l}\text { Evaluation du système de } \\
\text { formation professionnelle dans un } \\
\text { rapport annuel du gouvernement } \\
\text { (Berufsbildungsbericht). Ce rapport } \\
\text { s'appuie sur des données de } \\
\text { l'Institut fédéral pour la formation } \\
\text { professionnelle et est assorti d'une } \\
\text { prise de position de la commission } \\
\text { principale (Hauptausschu } \beta \text { ) de celui- } \\
\text { ci. Prises de position régulières des } \\
\text { instituts de recherche des } \\
\text { syndicats et du patronat. }\end{array}$ & Niveau fédéral \\
\hline
\end{tabular}




\begin{tabular}{|c|c|c|}
\hline $\begin{array}{l}\text { Contribution des organisations } \\
\text { professionnelles à la formation des } \\
\text { formateurs dont sont responsables } \\
\text { les chambres économiques, ainsi } \\
\text { qu'à la mise en place et à la } \\
\text { gestion de centres de formation } \\
\text { interentreprises, dont celles-ci } \\
\text { sont également responsables. Les } \\
\text { syndicats de salariés sont associés } \\
\text { à la gestion de ces centres. }\end{array}$ & $\begin{array}{l}\text { Travail permanent d'évaluation au } \\
\text { sein des organismes professionnels } \\
\text { et des fédérations syndicales par le } \\
\text { biais des liens avec les entreprises, } \\
\text { mais pas de pouvoir d'intervention } \\
\text { à ce niveau. }\end{array}$ & Niveau sectoriel \\
\hline $\begin{array}{l}\text { Forte implication des chambres } \\
\text { économiques dans la mise en } \\
\text { œuvre de l'apprentissage en } \\
\text { entreprise : elles sont responsables } \\
\text { des examens de fin } \\
\text { d'apprentissage, de la formation } \\
\text { des formateurs et de la gestion des } \\
\text { centres de formation inter- } \\
\text { entreprises. Les syndicats y sont } \\
\text { représentés dans des commissions } \\
\text { de formation professionnelle } \\
\text { (Berufsbildungsausschüsse). } \\
\text { L'enseignement scolaire s'effectue } \\
\text { dans les Berufsschulen, } \\
\text { établissements gérés par les } \\
\text { Länder. }\end{array}$ & $\begin{array}{l}\text { Contrôle de l'apprentissage en } \\
\text { entreprise par les chambres } \\
\text { économiques. En règle générale, } \\
\text { leurs conseillers en formation } \\
\text { (Ausbildungsberater), qui exercent } \\
\text { ce contrôle, n'interviennent } \\
\text { pourtant que lorsqu'apparaissent } \\
\text { des dysfonctionnements (plaintes } \\
\text { d'apprentis ou mauvais résultats } \\
\text { aux examens dans une proportion } \\
\text { inhabituelle). Les représentants } \\
\text { syndicaux dans les commissions de } \\
\text { formation des chambres peuvent } \\
\text { attirer l'attention par des prises de } \\
\text { position, mais n'ont pas le droit } \\
\text { d'agir. Ils peuvent notamment être } \\
\text { renseignés par des membres des } \\
\text { conseils d'entreprise. }\end{array}$ & Niveau régional \\
\hline $\begin{array}{l}\text { Les conseils d'entreprise } \\
\text { (Betriebsräte) ont un droit de } \\
\text { codécision dans la mise en œuvre } \\
\text { de l'apprentissage. Toutefois, il n'y } \\
\text { a pas de Betriebsräte dans toutes les } \\
\text { entreprises. }\end{array}$ & $\begin{array}{l}\text { Contrôle par les conseils } \\
\text { d'entreprise lorsqu'ils existent. }\end{array}$ & Niveau des entresprises \\
\hline
\end{tabular}

D’après : Wolfgang Streeck et al., Steuerung und Regulierung der beruflichen Bildung, Berlin, 1987.

12 Ce dispositif complexe de régulation à tous les niveaux comporte nombre de contraintes et lourdeurs puisqu'il exige beaucoup de temps et de moyens et qu'il nécessite une concertation permanente entre tous les partenaires. De ce fait, il n'évolue que lentement, notamment pour ce qui est de l'adaptation et de la modernisation des règlements de formation, qui relèvent d'une procédure longue et parfois quelque peu laborieuse.

13 Mais ce qui fait sa lourdeur garantit aussi sa stabilité et assure la nécessaire continuité des politiques de formation, lesquelles s'inscrivent par nature dans la durée. Cela garantit en outre sa légitimité sociale et surtout son efficacité, car des décisions et des orientations définies par consensus entre des interlocuteurs directement intéressés et 
compétents sont en général plus pertinentes, plus durables et mieux appliquées, puisque toutes les parties prenantes ont été associées à leur élaboration.

\section{L'école professionnelle, maillon faible du système}

14 Ce système comporte néanmoins, dans son architecture globale, un point faible, celui de l'articulation entre l'apprentissage en entreprise et l'école professionnelle. Il existe en général une bonne coopération entre les deux composantes du système dual, fondée sur une longue pratique de dialogue et de concertation. Notre enquête nous a fourni de multiples exemples d'un partenariat vivant et effectif. Mais on relève également, dans la plupart des secteurs, des frictions latentes entre l'entreprise et l'école professionnelle qui sont dues principalement :

- au fait que les programmes des Berufsschulen ne sont coordonnés avec les Ausbildungsordnungen que par des programmes-cadres nationaux (Rahmenpläne) assez généraux, qui peuvent être interprétés de façon assez différente selon les Länder ;

- à une certaine concurrence entre entreprise et école pour le partage du temps de travail de l'apprenti - c'est d'autant plus vrai que l'entreprise est plus petite et compte sur la contribution productive de ce dernier. On voit en outre s'exprimer fréquemment des appréciations divergentes sur les contenus et la qualité de la formation dispensés par l'autre partenaire. En particulier, les entreprises se plaignent moins du caractère général de la formation scolaire, que de sa qualité quant à l'acquisition des savoirs fondamentaux ;

- aux insuffisances propres des Berufsschulen : celles-ci apparaissent de plus en plus comme le parent pauvre du système éducatif : elles manquent de crédits et elles manquent de maîtres, en raison d'une crise de recrutement, liée elle-même à un déficit d'image par rapport aux établissements d'enseignement général. Il en résulte des vacances de postes et des absences non remplacées, des cours non rattrapés et des retards dans le respect des programmes, autant d'éléments qui sont perçus comme une perturbation et un manque d'efficacité par l'entreprise. En outre, la crise de recrutement entraîne également une baisse perceptible dans la qualification du corps enseignant, tandis que, sous l'effet d'un progrès technique rapide, le professionnalisme des formateurs d'entreprise, notamment dans les grandes entreprises, ne cesse de s'accroître. Cette "déconnexion rampante" de l'école professionnelle peut à terme devenir préoccupante, car elle risque de conduire progressivement à un grippage dans le dialogue et la coopération entre l'école et l'entreprise, menaçant ainsi l'équilibre général du système.

Certaines entreprises, notamment les plus grandes, échappent à ces problèmes soit parce qu'elles disposent, par statut dérogatoire, de leur propre Berufsschule et s'en félicitent (cas de Bayer par exemple) ${ }^{1}$, soit parce qu'au niveau local, par des aides complémentaires et un soutien logistique et pédagogique multiforme, elles exercent une influence bénéfique sur le fonctionnement général de l'école. La situation est également plus favorable lorsqu'il existe une certaine concentration géographique des activités : ainsi à Cologne, capitale de l'assurance, les compagnies d'assurance qui y ont leur siège central ont favorisé la mise sur pied d'une école professionnelle orientée vers cette activité et qui accueille leurs apprentis. Cela contribue à une coopération très étroite entre les formateurs, à la cohésion et à la qualité de la formation dispensée dans la branche. La situation est cependant nettement moins favorable dans les zones à moindre densité urbaine, où le tissu économique est hétérogène et où prédomine la petite et moyenne entreprise. Dans ce cas de figure relativement fréquent, le standard 
moyen de la Berufsschule tend à se déprécier. Les difficultés des Berufsschulen et les critiques dont elles font parfois l'objet dans les milieux professionnels illustrent en fait, de façon indirecte mais assez clairement, la prépondérance de l'entreprise dans le système dual et l'ascendant qu'elle exerce.

\section{Prépondérance de l'entreprise et consensus social}

$\mathrm{Au}$ total, deux éléments-clés assurent la cohérence et l'équilibre d'un système institutionnel aussi complexe :

\section{Le rôle central de l'entreprise}

Celle-ci est en fait la clé de voûte de l'édifice, car c'est à elle qu'incombe la responsabilité effective de la mise en œuvre de la formation. Cette responsabilité s'exerce à travers le contrat de formation (Ausbildungsvertrag), contrat codifié et standardisé, qu'elle signe avec l'apprenti qu'elle a elle-même recruté parmi les candidats ayant sollicité auprès d'elle une place d'apprentissage. Tout au long de sa formation, l'apprenti sera statutairement placé sous son autorité, y compris pour ce qui est du bon déroulement et de la réussite de sa formation scolaire. Dans le système allemand, l'alternance se fait donc exclusivement sous statut entrepreneurial.

En tant que maître d'œuvre de la formation, l'entreprise est responsable du respect de la Ausbildungsordnung, ce qui l'amène, le cas échéant, à devoir pallier elle-même les lacunes de la Berufsschule, afin d'assurer la réussite de l'apprenti. L'entreprise peut, de ce fait même, choisir la Berufsschule à laquelle elle envoie ses apprentis. Et surtout, c'est elle qui décide de former ou non et de demander son habilitation à devenir entreprise formatrice.

19 Enfin, l'entreprise est effectivement maître d'œuvre de la formation, parce qu'elle y affecte ses moyens financiers et humains propres. En contrepartie, elle dispose d'une autonomie effective dans l'organisation proprement dite de la formation, à charge pour elle de se conformer aux obligations et aux normes de la réglementation. A partir du moment où elle a décidé de devenir formatrice et s'en est vue reconnaître la capacité, l'entreprise s'accommode sans difficulté majeure du respect du cadre réglementaire. Le contrôle auquel elle est soumise est davantage un contrôle de conformité que d'opportunité et de contenu. En particulier, rien ne l'empêche d'innover, d'adapter et d'améliorer ses formations en permanence, en allant souvent bien au delà de la réglementation-plancher, en fonction de ce qu'elle estime être ses besoins propres en termes de qualification de sa main-d'œuvre future. En ce sens, dans le cadre réglementaire imparti, l'entreprise est souveraine dans l'ampleur et les modalités de mise en œuvre de son investissement-formation.

\section{Le consensus social}

L'entreprise ne peut cependant s'acquitter de cette tâche, laquelle est d'ailleurs un des principaux éléments qui la fait apparaître en Allemagne comme "entreprise citoyenne", que parce que le corps social lui reconnaît et lui confie cette responsabilité. La reconnaissance de la fonction formatrice de l'entreprise repose sur un consensus entre les pouvoirs publics et les partenaires sociaux. Il s'agit d'un consensus pragmatique que 
l'on pourrait qualifier de "pacte de qualification", dans lequel chaque partie trouve son compte: l'entreprise s'assure une main d'œuvre qualifiée adaptée à ses besoins, les syndicats parient sur la formation pour garantir un haut niveau de rémunération et une meilleure stabilité des emplois face aux évolutions techniques, les pouvoirs publics voient dans une insertion professionnelle efficace un facteur de stabilité sociale, de compétitivité et de croissance économiques.

Ce consensus est un fait de base, plus solide que la conflictualité apparente: qu'on pense aux négociations sur la refonte de l'Ausbildungsordnung de la métallurgie, qui progressent de manière décisive en 1984 , alors que les grèves sur la réduction du temps de travail battaient leur plein dans un climat d'agressivité extrême. De même, les polémiques sur la réforme du système de formation professionnelle de la fin des années 70 n'avaient pas empêché les négociations sur la refonte des qualifications dans le bâtiment de progresser (cf. chapitre IV).

Ce consensus s'exerce au niveau fédéral à l'occasion des refontes en question, mais il joue également un rôle important au niveau de l'entreprise par l'intermédiaire du conseil d'entreprise qui dispose d'un droit de contrôle, avec pouvoir de codécision, sur l'organisation de la formation professionnelle à ce niveau. Le conseil d'entreprise peut ainsi éviter les abus, faire valoir les droits des apprentis et veiller à la qualité de la formation.

Enfin, la dernière composante de ce consensus, et pas la moindre, puisqu'elle en est en fait le véritable ciment, c'est la légitimité dont jouit le système auprès des Allemands eux-mêmes, tant auprès des familles que des jeunes. Les uns et les autres voient dans l'apprentissage dual un système qui permet à la fois d'acquérir une qualification professionnelle solide, d'offrir au départ de bonnes chances d'insertion, puis par la suite, en fonction du mérite de chacun, de réelles possibilités de promotion professionnelle.

De ce point de vue, le consensus ne saurait être considéré que comme un élément contextuel déterminant, car il est tout autant le produit que le fondement du système. Ainsi, à notre sens, l'efficacité du système dual réside moins dans une aptitude particulière de la société allemande au consensus, que dans la capacité de ses responsables politiques et sociaux à définir ensemble, de façon évolutive et pragmatique, des règles du jeu cohérentes et opérationnelles, dont la légitimité se construit à l'épreuve des faits.

\section{NOTES}

1. Il faut préciser que les professeurs restent des diplômés d'Etat ayant un statut de fonctionnaires détachés et que, bien entendu, les programmes officiels sont respectés. 


\section{Une offre de formation différenciée, mais de qualité}

1 Fondé sur la libre initiative des entreprises, le système dual présente l'avantage essentiel lié à cet engagement volontaire de l'économie dans la formation: il est générateur de professionnalisme, ce qui profite tant aux employeurs (cf. aussi chapitre V) en termes d'efficacité économique, qu'aux salariés en termes d'insertion (cf. à ce sujet chapitre VI). Mais cet avantage ne peut pleinement jouer que grâce à une forte régulation du marché de l'apprentissage : non seulement les règlements de formation homogénéisent l'offre, mais encore les mécanismes institutionnels, décrits au chapitre précédent, et surtout l'esprit dans lequel les partenaires sociaux les utilisent depuis le milieu des années 70 ont permis une forte amélioration du niveau des formations et un développement de la polyvalence.

\section{Une offre de formation différenciée}

2 Comme nous l'avons vu, l'apprentissage dual repose fondamentalement sur un mécanisme de marché, puisque les entreprises décident librement si elles veulent former ou non. Cette décision dépend d'un calcul qui tient compte des coûts et des apports de la formation (cf. chapitre V), ceux-ci dépendant évidemment du type de spécialité dans laquelle il s'agit de former, mais devant être aussi considérés en termes de coûts d'opportunité. L'élément-clé est l'offre de main d'œuvre sur le marché du travail local. L'insuffisance de celle-ci prime tous les autres facteurs, car l'entreprise ne peut attendre que le système scolaire forme à sa place. De plus, les employeurs ont intérêt à éviter une situation de pénurie de main d'œuvre qui pourrait faire monter le niveau des revendications salariales ${ }^{1}$. 
Tableau 3. Repartition des apprentis par catégorie d'établissement

\begin{tabular}{|c|c|c|c|c|c|c|c|}
\hline \multirow{2}{*}{$\begin{array}{l}\text { Etablissements } \\
\text { de .... a ... } \\
\text { personnes (1) }\end{array}$} & \multicolumn{3}{|c|}{1970} & \multicolumn{3}{|c|}{1987} & $\begin{array}{l}\text { Taux } \\
\text { d'apprentissage }\end{array}$ \\
\hline & total & masculin & féminin & total & masculin & téminin & 1987 \\
\hline $1 \cdot 4$ & 10,3 & 7,5 & 14,9 & 7,1 & 5,4 & 9,2 & 6,0 \\
\hline $5 \cdot 9$ & 19,2 & 15,9 & 24,4 & 18,6 & 15,3 & 22,7 & 10,6 \\
\hline $10 \cdot 19$ & 14,0 & 14,3 & 13,4 & 15,7 & 16,0 & 15,4 & 9,8 \\
\hline $20 \cdot 49$ & 13,8 & 15,2 & 11,5 & 14,1 & 15,5 & 12,3 & 7,2 \\
\hline $50 \cdot 99$ & 8,8 & 9,5 & 7,6 & 8,7 & 9,2 & 8,1 & 5,8 \\
\hline $100 \cdot 199$ & 8,2 & 8,4 & 7,9 & 8,2 & 8,5 & 7,8 & 5,6 \\
\hline $200 \cdot 499$ & 9,7 & 9,9 & 9,4 & 10,9 & 10,9 & 10,9 & 5,8 \\
\hline $500-999$ & & & & 6,8 & 6,8 & 6,9 & 5,9 \\
\hline & 16,0 & 19,3 & 10,9 & & & & \\
\hline 1000 et plus & & & & 9,8 & 12,4 & 6,7 & 4,8 \\
\hline Total & 100,0 & 100,0 & 100,0 & 100,0 & 100,0 & 100,0 & 6,5 \\
\hline
\end{tabular}

(1) Le terme d'établissement est compris tous secteurs confondus (y compris professions libérales). Le nombre de personnes affiché inclut les apprentis.

(2) Part des apprentis dans le personnel de l'établissement en \%. Pour la catégorie 1-4 personnes le taux a été établi en excluant les établissements de 1 ou 2 personnes.

D'après : Berufsbildung in Wissenschaft und Praxis, 4/1990

3 Selon le recensement des lieux de formation fait en 1987 (qui inclut entreprises, mais aussi administrations et professions libérales), environ $20 \%$ des établissements ont une activité de formation initiale. Les établissements de 1 à 4 personnes ${ }^{2}$, bien que représentant la majorité des lieux de travail, ne réunissent que 7,1\% des apprentis. Par contre, dans les catégories de 10 à 199 personnes, 50 à $75 \%$ des établissements ont choisi de former. C'est là qu'on trouve près de la moitié des apprentis. Les établissements de plus de 1000 personnes forment à plus de $85 \%$. Mais compte tenu de leur nombre réduit et d'un taux d'apprentissage ${ }^{3}$ plutôt inférieur à la moyenne $(4,8 \%$ contre $6,5 \%$ ), ils ne drainent qu'à peine $10 \%$ des apprentis.

4 L'offre d'apprentissage est par ailleurs sensiblement différente sur le plan qualitatif selon le secteur et la profession dans le secteur, les deux pôles étant les formations administratives et commerciales $\mathrm{du}$ secteur banque/assurance qui sont intellectuellement les plus exigeantes et, à l'opposé, les formations du commerce de détail ou de la coiffure.

5 Ce critère de différenciation doit être en outre croisé avec celui de la taille de l'entreprise. On peut ici opposer schématiquement les petites entreprises, où la formation est plutôt intégrée dans le processus de production, aux grands établissements qui, en ce qui concerne les formations industrielles, disposent d'ateliers d'apprentissage. Dans ce dernier secteur, la formation s'est séparée de l'activité de production dans la mesure où elle est conçue et réalisée par un service propre avec un grand nombre de formateurs à plein temps. Une partie importante (en règle générale la première phase) de la formation en entreprise est effectuée dans un atelier d'apprentissage très bien équipé et permettant la mise en œuvre de méthodes didactiques élaborées. L'entreprise organise par ailleurs souvent un enseignement théorique qui complète celui de l'école professionnelle. 
6 En revanche, les petites entreprises du secteur manufacturier, même si elles ont installé des "coins d'apprentissage" (Lehrecken), intègrent très vite leurs apprentis dans le processus de production. Quand, dans l'artisanat, le seul formateur agréé est le maitre artisan, il est clair que les apprentis doivent souvent travailler sous la direction des ouvriers qui ne sont pas forcément préoccupés de pédagogie. La formation est par conséquent moins systématique et moins théorique. En conclure qu'elle est forcément plus mauvaise serait un peu rapide, car, dans la mesure où la division du travail y est moins poussée que dans une grande unité de production, la petite entreprise peut mettre l'apprenti dans des situations plus variées, donc lui apprendre plus rapidement une certaine forme d'autonomie et de polyvalence.

7 Il est vrai cependant que les petites entreprises attendent plus souvent des jeunes qu'ils soient rapidement une main-d'œuvre rentable. Les taux d'apprentissage évoqués plus haut montrent bien que, à la différence des grandes entreprises, qui, supportant des coûts de formation élevés, raisonnent en termes d'investissement-formation strictement calculé pour couvrir leurs besoins de qualification, les petites ont tendance à recruter plus d'apprentis qu'il n'est à cet égard nécessaire. Ainsi, on a pu observer dans le passé qu'en période de fléchissement conjoncturel la part relative de l'artisanat dans le total des jeunes en apprentissage a toujours sensiblement augmenté. Compte tenu de ces faiblesses ${ }^{4}$ qu'on ne peut négliger, car la majorité des apprentis se trouve dans des établissements de moins de 50 salariés, il est d'autant plus important que les formations soient réglées par des référentiels professionnels nationaux (Ausbildungsordnungen).

\section{Les règlements de formation (Ausbildungsordnungen) : vecteurs de diffusion d'une culture professionnelle}

8 Ces règlements comprennent la liste des tâches que l'ouvrier doit être en mesure d'effectuer une fois qualifié, un plan de formation tant systématique que chronologique, enfin la définition des épreuves de l'examen terminal. Ils fixent donc des exigences minimales de formation et opèrent une standardisation des professions, à laquelle n'échappe aucun secteur de l'économie.

9 Il est intéressant de noter qu'historiquement le principe des référentiels professionnels n'a nullement été imposé par l'Etat. L'idée d'homogénéiser les formations est née dès avant la Première Guerre mondiale au sein de l'industrie (la première prise de position en ce sens émane du "Comité allemand pour la scolarité technique" - DATSCH, Deutscher Ausschuß für Technisches Schulwesen, qui date de 1911) et a été mise en œuvre par l'industrie elle-même à partir des années 20. Pour un grand nombre d'entreprises, malgré l'émergence, au tournant du siècle, de formes d'organisation du travail déqualifiantes liées aux progrès de la mécanisation, les origines artisanales n'étaient pas oubliées et le métier restait une valeur de référence ${ }^{5}$. Par ailleurs, cette attitude s'explique aussi par la propension de l'industrie, fortement marquée à l'époque, à s'organiser pour limiter la concurrence - une tendance qu'illustrent aussi le développement des normes de fabrication et surtout la forte cartellisation.

10 Le système des Ausbildungsordnungen contribue de manière décisive au fonctionnement d'un marché dont on peut dire avec Burkhardt Lutz qu'il est "assez subtil". En effet, "la marchandise produite et achetée par les employeurs, la qualification, est liée à une 
personne, donc volatile. [...] L'entrepreneur-employeur fait un investissement en capital humain sans pouvoir assurer le retour d'investissement sur la personne même" ${ }^{6}$. En RFA, si le salarié quitte l'entreprise avant que son apprentissage soit amorti, l'employeur n'a pas tout perdu: grâce aux règlements de formation, qui assurent la diffusion d'une culture professionnelle homogène à l'ensemble de l'économie, il peut plus facilement le remplacer en embauchant un salarié formé par la concurrence. Dans la mesure où chacun peut espérer profiter de l'effort collectif auquel il participe, la formation peut véritablement être qualifiée d"“impératif catégorique".

Figure 3. Procédure d'élaboration des règlements de formation

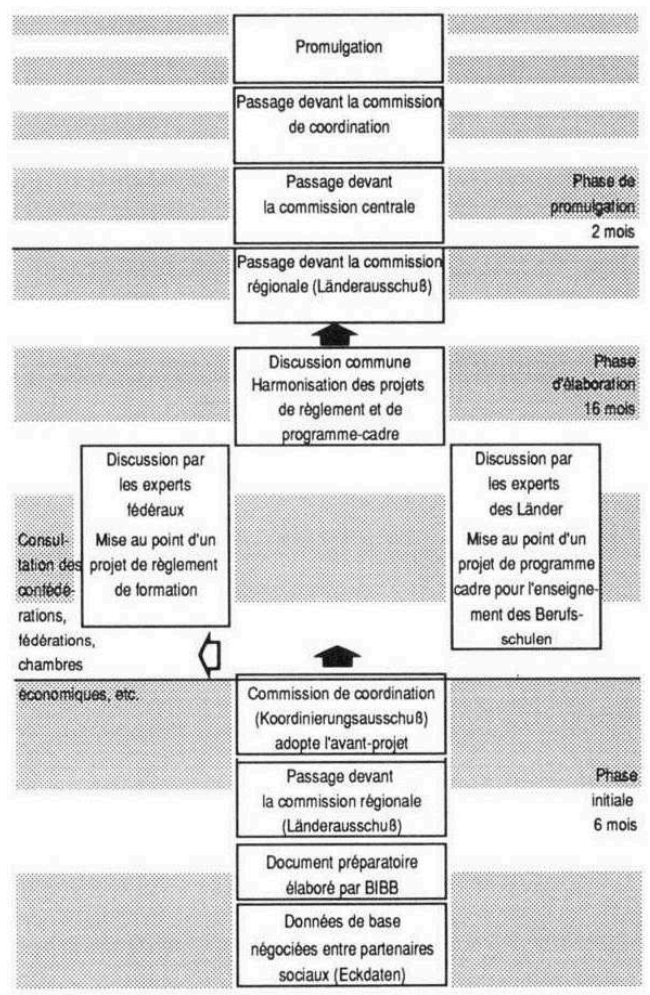

D'après : H. Benner : Ordnung der staatlich anerkannten Ausbildungsberufe. Berichte zur beruflichen Bildung, Heft 48, Berlin, 1982, p. 67.

L'élaboration des règlements de formation (Ausbildungsordnungen)

Il s'agit d'une procédure longue et complexe, car elle suppose l'intervention et l'accord d'un grand nombre d'acteurs. Y participent des représentants des Länder, des syndicats de salariés et des organisations d'employeurs (confédérations, mais aussi fédérations de la branche où le règlement de formation est en discussion), ainsi que du ministère fédéral de la Formation et de la Science et du ministère fédéral concerné (selon le cas le ministère de l'Economie ou de l'Agriculture). L'Institut fédéral pour la formation professionnelle (Bundesinstitut für Berufsbildung - BIBB) joue également un rôle central, non seulement en tant que pôle d'expertise, mais aussi comme cadre institutionnel. Discussions et votes se déroulent notamment au sein de sa commission centrale (Hauptausschuß), ou d'une sous-commission de celle-ci, la commission régionale (Länderausschu $\beta$ ), où sont représentés les partenaires sociaux, les autorités fédérales et les Länder. Une autre instance importante est la commission de coordination (Koordinierungsausschu $\beta$ ), 
où ne siègent que des représentants des ministères fédéraux et des ministères de l'Education des Länder.

Toute élaboration d'un nouveau règlement de formation ou refonte d'un règlement déjà existant, qui peut être demandée par les syndicats, le patronat ou le BIBB, suppose l'accord préalable de ces trois parties. S'ouvre alors une phase initiale qui dure habituellement 6 mois. Elle débute par une première négociation que le ministère fédéral concerné (le plus souvent le ministère de l'Economie) organise entre les partenaires sociaux, des membres du BIBB, des représentants des ministères fédéraux et de la conférence des ministres de l'Education régionaux (Kultusminister konferenz - KMK, une instance permanente, non prévue par la Constitution fédérale et ne remettant donc pas en cause l'autonomie des Länder en matière d'éducation, mais à laquelle ceux-ci participent volontairement pour coordonner leur politique dans ce domaine). Cette négociation doit permettre de dégager un accord sur cinq points fondamentaux (Eckdaten) : la désignation exacte de la profession, la durée de la formation, les principales caractéristiques de la qualification, la structure du cursus de formation et les étapes ultérieures du processus de négociation. A partir de cette trame, le BIBB rédige ensuite un document préparatoire, qui est soumis à la commission régionale (Länderauschu $\beta$ ) et au ministère fédéral concerné. C'est à ce dernier, qui détient le pouvoir réglementaire pour la formation en entreprise, qu'il incombe de rédiger la demande officielle de révision (ou d'élaboration) du règlement de formation en tenant compte de l'avis du Ldnderausschufi. Mais il faut l'accord préalable de la commission de coordination (Koordinierungsausschu $\beta$ ) avant que ne s'engage la phase d'élaboration proprement dite.

Cette phase d'élaboration, qui dure normalement 16 mois, comprend les étapes suivantes : mise au point parallèle, d'une part, d'un projet de règlement de formation entre les experts des partenaires sociaux et ceux du BIBB, d'autre part, d'un projet de programme-cadre pour les écoles professionnelles (Berufsschulen) au sein d'une commission ad hoc de la conférence des ministres de l'Education régionaux (KMK). Ce programme-cadre fixe les grands traits de l'enseignement des Berufsschulen, tout en laissant à chaque Land une marge d'interprétation conforme au fédéralisme éducatif. Les deux documents, dont le premier est d'abord soumis à l'appréciation des confédérations syndicales et patronales et, par leur intermédiaire, à celle de leurs fédérations concernées, aux chambres économiques et à certaines entreprises, sont ensuite discutés par les deux groupes d'experts siégeant ensemble. Enfin, ils sont examinés par la commission régionale et la commission centrale du BIBB.

Les ministères fédéraux (ministère de la Formation et de la Science et ministère de tutelle du secteur économique concerné) peuvent, dans un troisième temps, passer à la promulgation, processus qui dure généralement 2 mois, car il comporte une ultime consultation de la commission de coordination. Le programme-cadre pour les écoles professionnelles fait quant à lui l'objet d'une promulgation par les ministres de l'Education des Länder. Cependant, les deux textes sont aussi publiés au journal officiel fédéral.

11 Du point de vue du salarié, ce fonctionnement du marché facilite la mobilité. Bien que l'apprentissage à l'allemande s'appuie sur une formation largement assurée par et dans une entreprise, le salarié peut changer d'employeur en limitant le risque de 
déqualification s'il garde le même métier. Sur ce point, on est à l'opposé du système de formation sur le tas et "d'emploi à vie", largement pratiqué dans les grandes entreprises japonaises. Cela signifie que les patrons allemands sont moins assujettis que leurs concurrents nippons aux contraintes qu'impose l'organisation d'un marché interne du travail ${ }^{7}$. Il est vrai que ce mode allemand de gestion du personnel correspond aussi au mode d'organisation collective du salariat : l'Allemagne ne connaît pas le syndicalisme d'entreprise.

$12 \mathrm{Au}$ total, le principe des Ausbildungsordnungen exerce une contrainte vertueuse: contrainte pour l'entreprise, car elle est tenue d'aligner les contenus de son apprentissage sur un niveau général, indépendant de ses besoins immédiats; mais vertueuse, car constituant un garde-fou qui préserve les salariés de formations au rabais, et à ce titre facteur de légitimation essentiel du système dual. Cela dit, toute entreprise qui estime ne pas pouvoir supporter les coûts correspondant à cette exigence garde la liberté de ne pas faire de formation - tout comme inversement, rien n'empêche une entreprise d'offrir plus que le minimum du référentiel.

\section{L'évolution vers la polyvalence des qualifications}

Bien que l'élaboration de règlements professionnels nationaux conduise par définition à généraliser à partir des pratiques de diverses entreprises, le système des Ausbildungsordnungen peut sanctionner des spécialités très pointues. Ce sont entre autres les "professions parcellaires" (Splitterberufe), profils très étroits acquis par des apprentissages courts que critiquent les syndicats dès l'entre-deux-guerres.

pour peu qu'intervienne un élargissement des champs professionnels, le système des Ausbildungsordnungen est un vecteur de diffusion puissant du gain de polyvalence induit. Un tel processus s'est amorcé en Allemagne dès les années 60. Alors que la discussion sur les conséquences de 1'automation et la nécessité d'une formation professionnelle de base bat son plein, le besoin de qualifications plus larges apparaît en effet dans les grandes entreprises des secteurs technologiquement en pointe ${ }^{8}$.

La loi de 1969 sur la formation professionnelle (Berufsbildungsgesetz) a favorisé ensuite le mouvement vers la polyvalence en associant les représentants syndicaux à l'élaboration des référentiels. La procédure, qui, conformément à la complexité institutionnelle du système dual, doit assurer un triple équilibre entre le privé et le public, les salariés et le patronat, l'échelon fédéral et les régions, est certes d'une extrême lourdeur. Le référentiel suppose non seulement l'accord entre les partenaires sociaux, mais il implique aussi d'une part l'intervention des ministères de l'Education des Länder qui, en tant qu'autorité de tutelle des Berufsschulen, fixent les grandes lignes $d u$ cursus scolaire parallèle à l'apprentissage en entreprise, et d'autre part la mise au point minutieuse des programmes et du curriculum d'apprentissage avec l'aide des spécialistes de l'Institut fédéral pour la formation professionnelle (Bundesinstitut für Berufsbildung - BIBB).

16 En dépit de cette complexité, il ne faut pas perdre de vue que l'essentiel, à savoir l'initiative de refondre une Ausbildungsordnung et le contenu même du nouveau profil professionnel, dépend de l'accord des partenaires sociaux. Or, il est clair que les organisations de salariés plaident pour la polyvalence parce qu'elles veulent assurer à leurs membres la mobilité individuelle sans déqualification, mais aussi améliorer le 
statut professionnel en augmentant le niveau de formation. Il n'est donc pas étonnant qu'à l'intérieur de ce cadre la refonte d'un grand nombre d'Ausbildungsordnungen (à l'entrée en vigueur des règlements en cours de promulgation, 246 des 374 professions actuellement reconnues, soit $2 / 3$, ont été redéfinies depuis $1969^{\circ}$ ) ait permis, par la volonté même des partenaires sociaux, un développement de la polyvalence.

\section{Le bâtiment}

17 Les premiers - et les plus spectaculaires - changements ont été opérés dans le bâtiment. La nouvelle organisation des formations, promulguée en 1974, est aujourd'hui encore la seule à intégrer dans un schéma commun la majorité des métiers d'une branche, artisanat et industrie confondus. Elle prévoit une spécialisation progressive en trois étapes. La formation de base commune occupe toute la première année, la distinction de trois grandes disciplines (terrassement - Tiefbau, construction au dessus du sol - Hochbau, et aménagement - Ausbau) s'opérant seulement en deuxième année. Enfin, après le passage d'un examen éliminatoire, une spécialisation fine dans l'un des 14 métiers qu'intègre la réforme constitue la phase terminale de formation, qui dure 9 mois et se clôt par le passage du certificat professionnel (cf. figure 4). L'examen en fin de deuxième année opère une sélection, ce qui conduit à qualifier ce modèle de formation par niveaux (Stufenausbildung). 
Figure 4. Formation par niveau pour 14 professions du bâtiment

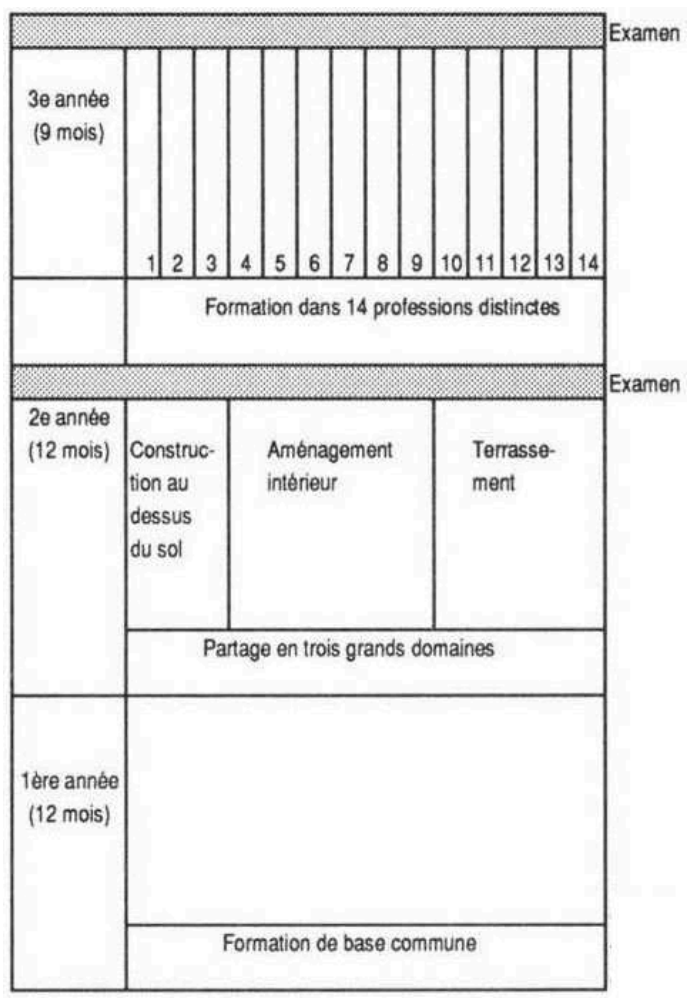

1 Maçon
2 Constructeur de béton et de béton armé
3 Constructeur de cheminées et de foyers
4 Charpentier
5 Façonneur en dalles de béton et briquetterie
6 Stucateur
7 Carreleur
8 Poseur d'enduits de sol
9 Monteur en isolation
10 Monteur d'éléments décoratifs ou de protection acoustique
11 Constructeur de routes
12 Poseur de conduites
13 Constructeur de canaux et de canalisations
14 Constructeur de puits

D'APRÈs : W. STREECK : STEUERUNg UND REgULIERUNg..., BERLIN, 1987.

Parallèlement, le bâtiment se singularise en mettant en place un système de financement collectif des dépenses de formation, au moment même où la confédération patronale rejette formellement les propositions du gouvernement social-démocrate/ libéral allant dans ce sens. Mais dans une branche qui comporte un grand nombre de petites entreprises, le patronat lui-même ne voit pas d'autre voie pour assumer les conséquences $\mathrm{du}$ principe de spécialisation progressive. La mutualisation des ressources permet en effet de mettre en place et de faire fonctionner rapidement un grand nombre de centres de formation inter-entreprises, qui peuvent seuls dispenser les enseignements du tronc commun. De plus, les fonds, collectés et gérés par les caisses sociales de la branche, permettent de rembourser aux employeurs une partie du manque à gagner occasionné par la réforme. En effet, pendant la première année de formation, la contribution de l'apprenti à la production est des plus réduites, puisque, entre le centre d'apprentissage inter-entreprises et la Berufsschule, il ne passe en tout guère plus de 6 semaines dans son entreprise. 
19 Malgré les contraintes considérables que la réforme impose, on comprend que les patrons du bâtiment aient trouvé un intérêt à la polyvalence et aux centres interentreprises. La très étroite spécialisation des professions de la branche s'était toujours mal accommodée de la nécessité pour les différents corps de métier de coopérer de façon flexible sur un chantier. De plus, compte tenu du caractère parfois spécialisé des travaux à effectuer et du fait des aléas de la météo, il était difficile de pratiquer l'apprentissage sur le tas en respectant un plan de formation. Quant au financement collectif, outre les raisons mentionnées plus haut, il se justifie aussi par l'importante fluctuation de la main-d'œuvre, qui empêche souvent à une seule entreprise d'amortir ses frais de formation. Enfin, au début des années 70, sévissait une crise de recrutement très sérieuse sur le marché de l'apprentissage, à laquelle les partenaires sociaux, traditionnellement coopératifs dans cette branche, se sentaient tenus de faire face de concert $^{10}$.

\section{La transformation des métaux et lélectrotechnique industrielles}

20 La situation est différente dans la transformation des métaux et l'électrotechnique, où patronat et syndicat, qui ont des rapports traditionnellement plutôt tendus, s'opposent au départ sur deux points fondamentaux. L'IG Metall ne veut pas entendre parler d'un système par niveaux (Stufenausbildung) comme dans le bâtiment, parce qu'il implique pour une partie des apprentis l'échec en cours de route, donc un statut professionnel dévalué. En outre, il existe un désaccord profond sur l'appréciation des besoins de qualification futurs, le patronat prévoyant une différenciation et une déqualification partielle, ce qui le conduit, à l'inverse du syndicat, à réclamer des formations très éclatées. La conclusion d'un accord après de très difficiles négociations n'en a que plus de valeur.

21 S'agissant de la métallurgie industrielle, où les référentiels datent des années 40 et même pour certains des années 30, les négociations sont longues et difficiles. Démarrées en 1975, elles n'aboutissent à un accord de principe qu'à la fin de 1978 et ne se traduisent par l'entrée en vigueur de nouvelles Ausbildungsordnungen qu'en... 1987 ! Le résultat n'en est pas moins remarquable, car il représente bien davantage que le plus petit commun dénominateur des deux points de vue initiaux. Certes, l'éventail des spécialités définies, qui reste relativement large, reflète bien un compromis entre les positions patronales et syndicales; mais sur la structure même des formations, l'entente aurait été impossible si les industriels de la métallurgie n'avaient pas entre temps changé de stratégie et décidé de parier sur des qualifications élargies.

Cette option reflète clairement un choix anti-taylorien: pour les patrons, il importe désormais qu'au delà de la simple exécution des tâches l'apprentissage développe les capacités d'analyse, de planification et de contrôle des opérations de production. En d'autres termes, les nouveaux référentiels valorisent l'autonomie et la responsabilité dans le travail. Les syndicalistes sont d'autant plus prêts à accepter cet objectif qu'ils en espèrent une plus grande stabilité de l'emploi, une réduction des risques en cas de mobilité, mais aussi une meilleure capacité des ouvriers à revendiquer un droit de cogestion sur le choix des techniques et l'organisation du travail. 
Figure 5. Nouvelles professions de la transformation des métaux - industrie

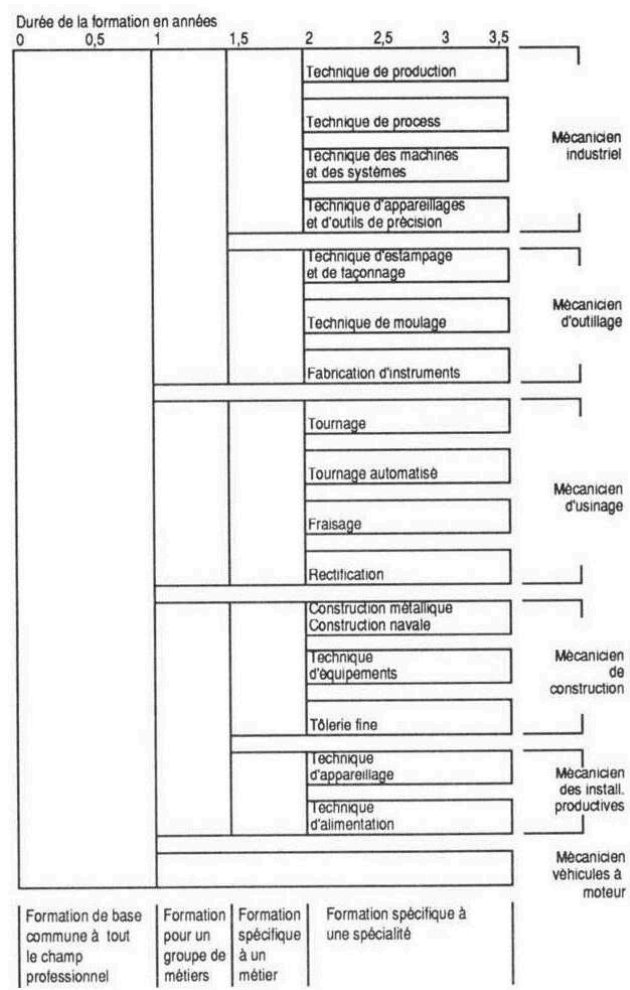

D'APRÈS : IG-METALL (ÉD.) : NEUE BERUFE, ANDERES LEMEN. SCHRIFTENREIHE DER IG-METALL HEFT 111, FRANCFORT 1987, P.21

Figure 6. Nouvelles professions de l'électronique - industrie

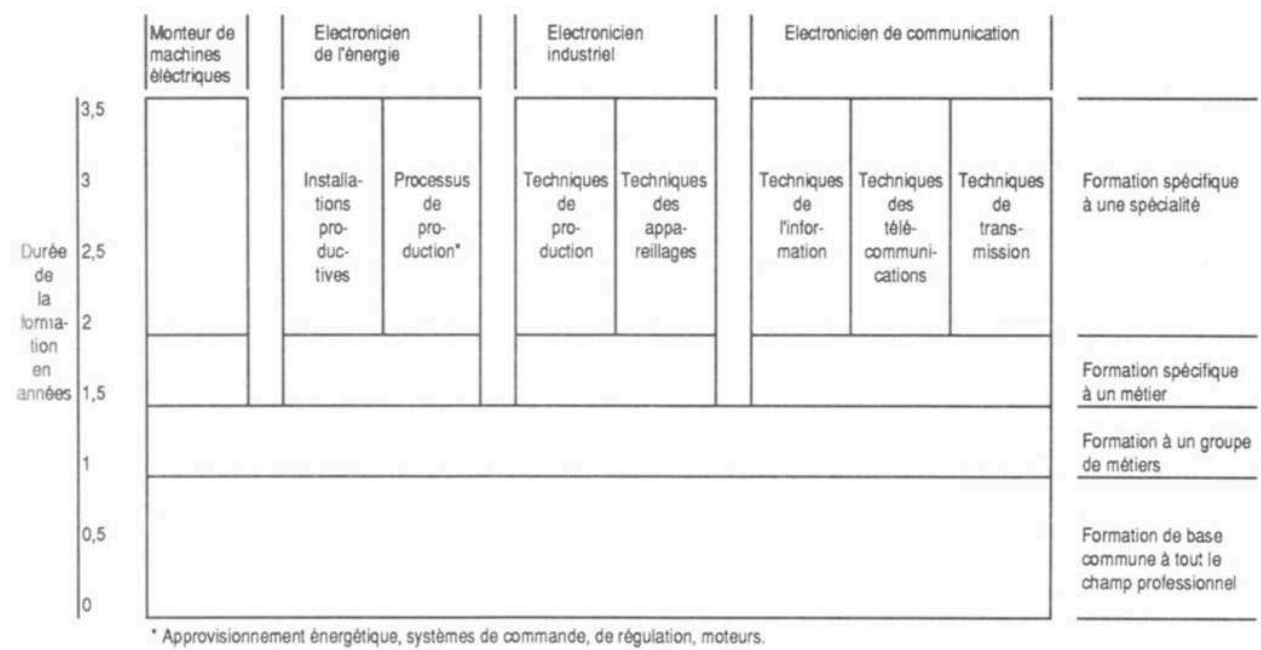

D'après : H. Benner : Ordnung der staatlich anerkannten Ausbildungsberufe. Berichte zur beruflichen Bildung, Heft 48, Berlin, 1982, p. 22.

Quels que soient les intérêts en partie divergents qui réunissent les partenaires sociaux autour de la nouvelle formule, la définition de la qualification d'ouvrier professionnel qui figure dans le protocole d'accord de 1978 sur les caractéristiques fondamentales 
(Eckdaten) du nouveau référentiel se lit comme un manifeste pour la polyvalence. Selon le texte, la qualification d'ouvrier professionnel permet :

- d'exercer la profession apprise dans différentes entreprises et différentes branches, ainsi que le cas échéant des activités d'ouvrier professionnel de nature voisine, après acquisition des savoir-faire manquants ;

- de s'adapter de façon flexible à des structures de travail, des méthodes de production et des technologies nouvelles, avec l'objectif de préserver la qualification professionnelle ;

- de participer à des mesures de formation permanente, continue et de recyclage pour préserver la qualification professionnelle et la mobilitée ${ }^{11}$.

Sur une telle base, la réorganisation des professions aboutit à une formation opérant une spécialisation progressive, mais, à la différence du bâtiment, sans examen intermédiaire sélectif. Après un tronc commun d'1 an, viennent 6 mois de formation plus spécifique, préparant à un groupe de professions (ainsi, les contenus sont par exemple communs à la mécanique industrielle et la mécanique-outil ou à la mécanique de construction et la mécanique des installations productives). La troisième phase, de 6 mois également, est propre à chacune des 6 professions, tandis que la spécialisation ultime à l'intérieur de celles-ci s'effectue dans l'an et demi qui reste. Toutes les professions concernées par la refonte ont donc une formation de 3 ans et demi. Les 37 professions "monovalentes" d'ouvrier qualifié (Monoberufe) qui existaient initialement sont remplacées par 6 professions polyvalentes, qui, mise à part la mécanique automobile, regroupent selon les cas, 3 ou 4 spécialisations. Et même si l'on considère comme le patronat que le profil professionnel donné par la formation est finalement la spécialité acquise pendant les 18 derniers mois d'apprentissage, et non la profession comprise au sens large, le gain de polyvalence saute aux yeux, puisqu'on passe de 37 à 17 subdivisions pour le champ professionnel qualifié de la métallurgie (cf. figure 5). Ceci dit, 14 formations d'une durée de 2 ans, qui débouchent sur un statut d'ouvrier semi-qualifié (Anlernberuf), ne sont pas remises en cause par la réforme.

Après l'avancée du protocole d'accord de 1978, les principes acquis pour les professions industrielles des métaux, peuvent être appliqués rapidement à celles de l'industrie électrotechnique, qui relèvent des mêmes organisations patronales et syndicales. Les Ausbildungsordnungen, qui sont également promulguées en 1987, obéissent à la même logique que celles de la métallurgie : des 12 "monoqualifications" d'avant la réforme, on passe à 4 professions regroupant au total 8 spécialisations (cf. figure 6).

\section{La transformation des métaux et l'électrotechnique artisanales}

Enfin, les solutions adoptées dans l'industrie sont reprises bientôt dans l'artisanat, tant pour la transformation des métaux que pour l'électrotechnique. Le regroupement des professions y est beaucoup moins radical, voire quasiment inexistant (de 19 à 17 dans le travail des métaux, maintien du même nombre dans l'électrotechnique), ce qui s'explique à la fois par les traditions et par l'obligation, à moins de recourir à un acte législatif, de respecter la liste des professions telle qu'elle est inscrite dans le code de l'artisanat. L'essentiel est cependant l'adoption du tronc commun et de la spécialisation progressive, qui constituent une modernisation considérable. On notera que la régulation de l'entrée en apprentissage par les mécanismes de marché a été ici particulièrement favorable à la diffusion de la polyvalence. En effet, dans un contexte démographique déjà favorable, les responsables patronaux se sont laissés convaincre de 
l'opportunité d'une réforme au moins autant par la crainte que "leurs" candidats à l'apprentissage soient captés par des formations d'autres branches réformées, donc plus attrayantes, que par la conscience des nécessités de l'évolution technique. C'est la concurrence, notamment des professions de l'industrie, qui a créé un effet d'entraînement ${ }^{12}$.

Figure 7. Nouvelles professions de la transformation des métaux - Artisanat

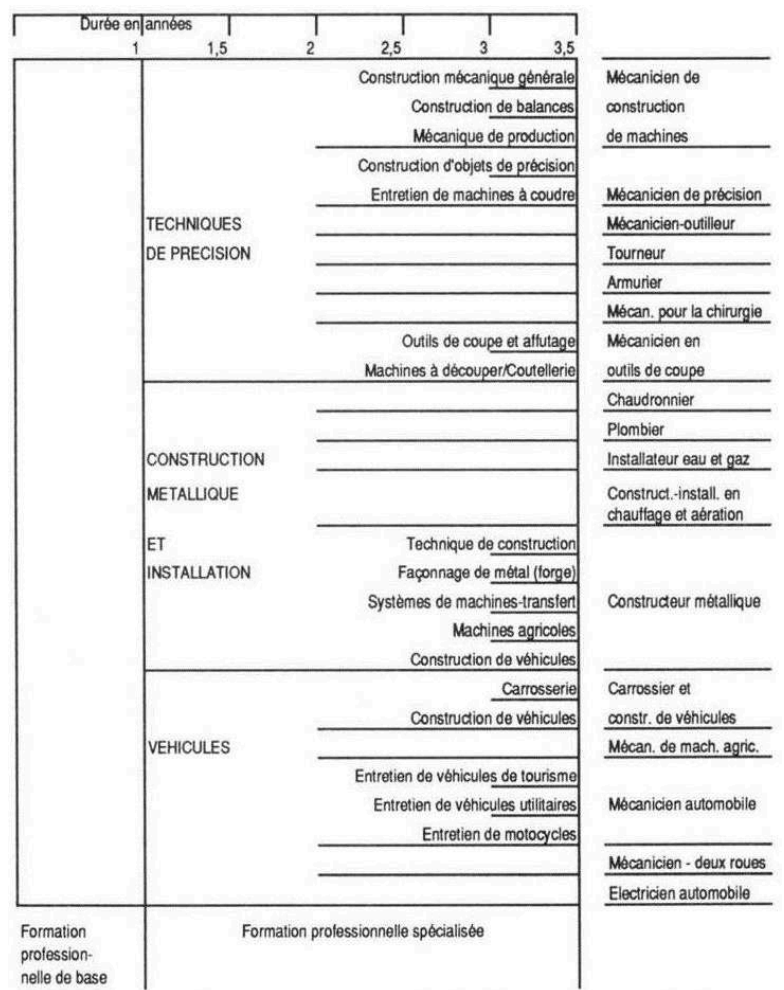

D'après : K. Stratmann/M. Schlösser : Das Duale System der Berulsausbildung. Francfort s.M., 1990, p. 273. 
Figure 8. Nouvelles professions de l'électronique - Artisanat

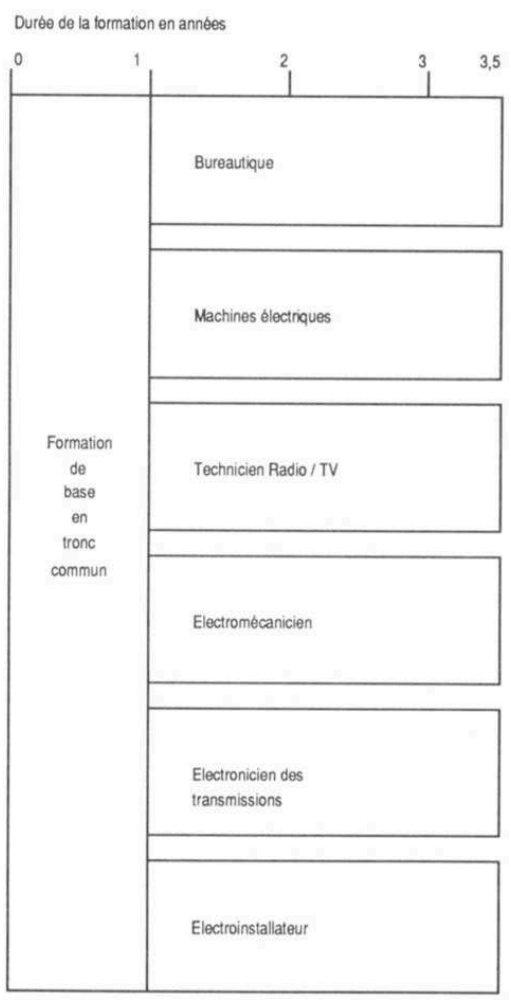

D'après : IG Metall (éd.), Elektrohandwerk - Neue Berufe. Arbeitshilfen, Heft 2. Düsseldorf, 1988, p. 9.

L'évolution dans les services et les professions de bureau montre bien que le mouvement amorcé dans le bâtiment et la métallurgie est en train de s'étendre à l'ensemble de l'économie. Face à une évolution technologique rapide, qui contraint à rénover des référentiels pourtant en partie récents ${ }^{13}$, l'économie définit des profils de qualification dont la caractéristique est d'être plus larges et d'intégrer la dimension de l'adaptation. Les formulations du protocole d'accord patrons/syndicats de mai 1988 sur la refonte des Ausbildungsordnungen professions de bureau (services publics exclus) sont particulièrement caractéristiques : il y est question de compétences professionnelles (connaissances et méthode), mais aussi de compétences personnelles et sociales, seule la combinaison des unes et des autres assurant l'autonomie professionnelle. L'accent mis sur ce que sociologues ou pédagogues appellent selon les cas "savoir-faire extrafonctionnels", "qualifications transdisciplinaires" ou plus souvent "qualifications-clés" (Schlüsselqualifi-kationen) montre bien qu'on tourne le dos à une démarche taylorienne pour miser, dans la recherche de la productivité maximale, sur la polyvalence.

L'idée souvent avancée par certains et complaisamment reprise en France selon laquelle le système dual allemand resterait marqué par une spécialisation professionnelle très poussée d'inspiration artisanale, puis taylorienne est donc non seulement contredite par la logique institutionnelle du système, mais également par l'analyse concrète des contenus des professions.

29 Ce choix de la polyvalence, particulièrement important dans la perspective de la réorganisation du travail dans l'industrie, a permis en outre de ressouder le consensus des partenaires sociaux sur l'apprentissage en entreprise, qui avait été fortement ébranlé dans le courant des années 70 . Compte tenu de l'importance primordiale de ce 
consensus pour le bon fonctionnement du système dual, on peut même dire que c'est en grande partie grâce à la rénovation des référentiels professionnels entreprise depuis le début des années 80 que le modèle allemand de formation professionnelle a retrouvé stabilité et légitimité.

On n'oubliera pas cependant que l'élévation du niveau de la formation professionnelle et l'élargissement des profils de qualification nécessitent un effort soutenu des entreprises. Les nouveaux référentiels ont d'ailleurs dissuadé certaines d'entre elles de continuer à former. Pour les PME qui ne disposent pas toujours de toutes les techniques requises, la solution peut être le recours aux centres inter-entreprises. Leur nombre a pu considérablement augmenter ces dernières années, notamment grâce au soutien des pouvoirs publics ${ }^{14}$.

\section{NOTES}

1. Pour l'influence de la politique de formation sur le fonctionnement du marché du travail, cf. Burkhardt Lutz, in: Martine Möbus/Eric Verdier (dir.), Le système de formation professionnelle en RFA, résultat de recherches françaises et allemandes, Paris (CEREQ: Collection des études $n^{\circ} 61$, février 1992, p. 141 sq.)

2. Dans cette statistique, le terme de "personne" inclut les apprentis éventuels. Pour tous les chiffres qui suivent, cf. Rudolf Werner, "10\% der Auszubildenden in Großbetrieben", in :Berufsbildung in Wissenschaft und Praxis, 4/1990, p. 35.

3. Le taux d'apprentissage est le rapport du nombre d'apprentis au chiffre total du personnel.

4. Dans les années 70, les abus ont suscité des protestations d'apprentis qui ont même été jusqu'à constituer un mouvement organisé.

5. C'est aussi pourquoi le discours sur les fonctions sociales du système dual est longtemps resté rétrograde: jusqu'au début des années 60 , beaucoup de pédagogues et de responsables économiques continuaient de présenter le choix de la profession comme l'expression d'une vocation pour un métier (les deux notions sont exprimées en allemand par le même mot : Beruf) et donc le choix d'une position dans l'Ordre social. Or, il est clair qu'à l'époque l'orientation professionnelle répondait déjà de plus en plus à des critères pragmatiques et utilitaristes, d'autant que l'accélération de la modernisation conduisait déjà un nombre croissant de salariés à changer une ou plusieurs fois de profession au cours de leur vie active.

6. Cf. B. Lutz, in: Möbus/Verdier, op. cit.

7. Pour une approche plus théorique du fonctionnement des marchés "professionnels" du travail et du cas de la RFA, système "mixte", dans la mesure où existent aussi des éléments concourant à la formation de marchés internes, cf. Werner Sengenberger, Struktur und Funktionsweise von Arbeitsmärkten, die Bundesrepublik Deutschland im internationalen Vergleich, Francfort s. M./New York, 1987, 126 sq. et 180 sq.

8. L'étude comparative du LEST d'Aix-en-Provence et de l'ISF de Munich sur le système de formation et l'organisation $d u$ travail en France et en Allemagne (Burkhardt Lutz, "Bildungssystem und Beschäftigungsstruktur in Deutschland und Frankreich. Zum Einfluß des Bildungssystems auf die Gestaltung betrieblicher Arbeitskräftestrukturen", in: Hans-Gerd Mendius et al., Betrieb, Arbeitsmarkt, Qualifikation, Francfort s. M., 1976 ; et Marc Maurice/François Sellier/Jean-Jacques Silvestre, Politique d'éducation et organisation industrielle en France et en 
Allemagne, Paris, 1981), dont les données empiriques avaient été collectées en 1971-72, diagnostiquait déjà une polyvalence des ouvriers qualifiés plus grande en Allemagne qu'en France.

9. Les chiffres cités ici (qui proviennent de Bundesministerium für Bildung und Wissenschaft, Berufsbildungsbericht 1992, Bad Honnef, 1992, p. 91) concernent les professions donnant une pleine qualification (gelernt et non angelernt), pour lesquelles, selon la jurisprudence, une formation de plus de deux ans est nécessaire. A peu près $96 \%$ des apprentis du système dual sont aujourd'hui formés dans des professions dont les règlements ont été modernisés.

10. Pour cette analyse, cf. Wolfgang Streeck et al., Steuerung und Regulierung der beruflichen Bildung. Die Rolle der Sozialparteien in der Ausbildung und beruflichen Weiterbildung in der Bundesrepublik Deutschland, Berlin, 1987.

11. Cf. Karlwilhelm Stratmann/Manfred Schlösser, Das duale System der Berufsbildung. Eine historische Analyse seiner Reformdebatten, Francfort s. M., 1990, p. 261.

12. Pour cette analyse, cf. Stratmann/Schlösser, op. cit. ; Streeck, op. cit. et Ernst Neumann, "Neuordnung der Berufsausbildung in den Metall- und Elektroberufen", in: Berufsbildung, 45 (1991)7/8.

13. Les Ausbildungsordnungen des principales professions administratives et commerciales avaient été révisées à la fin des années 70 et au début des années 80 .

14. On en compte actuellement près de 600 . Ces lieux de formation sont en règle générale sous la responsabilité des chambres économiques ou des syndicats patronaux, mais peuvent recevoir des subventions publiques pour peu qu'ils soient cogérés par des représentants syndicaux. Cf. WolfDietrich Greinert, Das duale System in der Berufsausbildung in der Bundesrepublik Deutschland, Struktur und Funktion, Eschborn, 1992. 


\section{Financement et coût de la formation dans le système dual}

1 La dualité du système, qui combine une formation dispensée en milieu scolaire au sein de la Berufsschule et une formation dispensée dans l'entreprise d'accueil de l'apprenti, se retrouve dans la mixité du financement Etat/entreprises. Néanmoins, dans ce financement mixte, l'entreprise supporte de loin la plus grande part de l'effort.

\section{Répartition de l'effort de financement}

2 Au titre de l'obligation scolaire jusqu'à 16 ans et, au delà de cet âge, du droit de tout apprenti de continuer à bénéficier d'une formation générale pendant toute la durée de sa formation (loi de 1969), le financement de la formation scolaire incombe naturellement à la puissance publique, en l'occurrence aux Länder qui, dans le cadre du fédéralisme, détiennent en la matière une compétence exclusive.

3 Le financement de la formation dispensée en entreprise, quant à elle, incombe logiquement à l'entreprise formatrice qui, sauf cas particuliers (jeunes en difficulté et aides spécifiques aux PME pour la création de centres inter-entreprises), l'assure intégralement. De même qu'il n'y a pas d'obligation pour l'entreprise de former des apprentis, le financement de la formation se fait sur une base strictement volontaire, hors de tout mécanisme de financement collectif socialisé ou para-fiscal.

4 Sur cette base juridique de volontariat intégral, il est par conséquent difficile de chiffrer l'effort global que les entreprises allemandes consacrent à la formation initiale (la difficulté est identique pour la formation continue qui repose sur le même principe). On ne dispose que d'enquêtes menées auprès des entreprises formatrices et d'estimations réalisées tous les 2 ou 3 ans par les organismes consulaires ou patronaux. L'Institut fédéral pour la formation professionnelle (BIBB) mène actuellement une enquête nationale systématique sur l'effort de formation des entreprises, mais celle-ci est encore inachevée.

5 La répartition et les caractéristiques respectives des séquences de formation permettent cependant de mettre en évidence une clé de répartition de l'effort national au niveau global. Le temps passé à la Berufsschule représente en règle générale le tiers 
de la durée totale de l'apprentissage, mais comme l'enseignement y est de caractère général et plus théorique, il est en moyenne moins coûteux en frais d'équipement et de fonctionnement que la formation technique dispensée en entreprise, de sorte que la répartition globale Etat/entreprises s'établit autour d'un rapport de 1 à 5 .

D'après les dernières estimations disponibles, l'effort global de formation initiale des entreprises (ouest-)allemandes s'établissait à 29,3 milliards de DM (100 milliards de FF) en 1991, ce qui représente $1,12 \%$ du PNB. De leur côté, les collectivités publiques (Länder et communes) ont consacré la même année 9,3 milliards de DM à la formation professionnelle dans le cadre scolaire ${ }^{1}$, soit un peu plus de $21 \%$ de l'effort global de formation professionnelle initiale. Pour l'ensemble de l'économie, tous secteurs confondus, le coût annuel moyen d'un apprenti s'établissait en 1991 à environ 22000 DM, dont $18000 \mathrm{DM}$ pour l'entreprise d'accueil et à $4000 \mathrm{DM}$ pour les collectivités publiques.

\section{La contribution de l'Etat}

7 Dans la partie scolaire placée sous la tutelle exclusive des Lander, l'effort est supporté concurremment par les collectivités publiques régionales et locales. Le ministère de l'Education du Land assure intégralement la rémunération (et les retraites) des enseignants des Berufsschulen et participe, sous forme d'une contribution complémentaire calculée au prorata des effectifs, au financement des équipements et $\mathrm{du}$ fonctionnement matériel des établissements, lequel est de la responsabilité principale des communes et/ou des cantons.

Cette responsabilité directe des collectivités locales dans l'équipement, l'entretien et le fonctionnement matériel des établissements a fait ses preuves pour la bonne tenue des écoles, même si la situation est moins favorable dans les grandes agglomérations où la charge financière est importante. En revanche, des problèmes sérieux se posent par suite d'arbitrages budgétaires défavorables à l'enseignement professionnel au niveau régional (Länder) : les établissements manquent de postes et surtout ont de plus en plus de difficultés à les pourvoir, car le statut de professeur de l'enseignement technique demanderait à être revalorisé.

Indépendamment de sa responsabilité financière directe pour la partie scolaire de la formation, l'Etat participe au financement de la formation professionnelle initiale par l'intermédiaire de différentes actions de soutien complémentaires. Ces aides s'adressent d'une part aux entreprises en vue d'améliorer le dispositif de formation existant et prennent la forme de subventions à la création de centres de formation inter-entreprises pour les PME (117 millions de DM en 1990 par l'intermédiaire du ministère fédéral de la Formation et de la Science, BMBW) ou à la création de places ou de cursus d'apprentissage dans l'artisanat (environ 68 millions de DM distribués aux PME en 1990 par l'intermédiaire du ministère fédéral de l'Economie). L'Etat apporte d'autre part un soutien spécifique individuel aux apprentis (bourses d'internat, indemnités de frais de transport, etc.) et à certaines catégories de jeunes en difficulté sous forme d'aides à la formation professionnelle dans le cadre de programmes d'insertion par l'intermédiaire de l'office fédéral du travail (Bundesanstalt fur Arbeit). Ces aides ont atteint un montant total de 1 milliard de DM en 1991 pour les Länder de l'Ouest. 


\section{Les dépenses de formation de l'entreprise}

10 L'entreprise supporte de son côté l'intégralité des dépenses engagées par la formation des apprentis effectuée en son sein, ce qui inclut, par importance décroissante :

\section{La rémunération des apprentis}

11 Elle est fixée contractuellement par la convention collective de la branche et varie selon les secteurs. Elle augmente également au cours des années de formation. La rémunération moyenne, tous secteurs et années de formation confondus, s'élevait à 838 DM en 1991, ce qui recouvrait un large éventail, allant de 503 DM pour l'apprenticoiffeur, à 959 DM pour l'apprenti-mécanicien industriel, 1240 DM pour l'apprentiagent d'assurance et à 1322 DM pour l'apprenti-maçon. Ici joue à la fois le niveau de rémunération moyen de la branche (la rémunération de l'apprenti est de l'ordre de 30 à $40 \%$ du salaire conventionnel de base) et le degré de rareté des apprentis dans des branches moins attractives, lesquelles cherchent à attirer les candidats par des rémunérations plus élevées, comme c'est le cas dans le bâtiment. Les rému-nérations augmentent d'environ $10 \%$ par année de formation, des suppléments sont parfois accordés aux apprentis de plus de 18 ans. Ces rémunérations sont considérées comme des dépenses normales de personnel et supportent les charges sociales de droit commun (environ $38 \%$ du salaire de base) payées en Allemagne pour moitié par le salarié et pour moitié par l'employeur. Le poste rémunération des apprentis, charges sociales comprises, représente en moyenne la moitié de la dépense de formation de l'entreprise. Dans les professions commerciales ou tertiaires, où les dépenses en équipement et matériel sont moins élevées que dans l'industrie et où les apprentis sont souvent mieux payés du fait d'un niveau de formation plus élevé (les apprentis y sont souvent en majorité bacheliers), la part relative à la rémunération des apprentis s'élève jusqu'à $75 \%$ de la dépense de formation.

\section{La rémunération des formateurs de l'entreprise}

Il s'agit presque toujours exclusivement de la rémunération des formateurs à temps plein, les chargés de formation ou tuteurs à temps partiel exerçant généralement leur activité de formateur dans le cadre de leur fonction principale, sans que cela donne nécessairement lieu à des primes ou suppléments spécifiques (ces fonctions de tuteurs sont cependant parfois prises en compte pour les promotions).

Les salaires des formateurs à temps plein sont censés tenir compte d'une qualification spécifique et reconnue de formateur, venant en plus d'une qualification et d'une expérience professionnelles effectives de contremaîtres (Meister) d'au moins 5 ans, et sanctionnée par un brevet spécifique de formateur. Les salaires des formateurs sont également fixés dans la convention collective et s'échelonnent entre 4000 et $5000 \mathrm{DM}$ nets de charges sociales. Cela les situe à un niveau comparable à celui des traitements des professeurs fonctionnaires des Berufsschulen, lesquels ont cependant pour la plupart une formation de niveau universitaire et des horaires moins contraignants (22 ou 24 heures hebdomadaires). Néanmoins, dans les grandes entreprises, les formateurs exerçant des fonctions de coordination perçoivent des rémunérations sensiblement plus avantageuses qui les placent tout en haut de l'échelle des contremaîtres (Meister) et 
qui peuvent dépasser les $6000 \mathrm{DM}$, avantages extra-conventionnels non compris. A ces rémunérations nettes viennent également s'ajouter pour l'entreprise les charges sociales habituelles. En moyenne, la rémunération des formateurs représente environ $20 \%$ des dépenses de formation.

\section{Les frais de formation}

Les frais de formation, c'est-à-dire les frais d'installation (locaux), d'équipement (machines et outillages), les fournitures (énergie, matières premières) ainsi que les frais généraux divers (entretien, maintenance, administration) constituent les $30 \%$ restants. Dans le cas des formations industrielles "pointues" faisant appel à des technologies sophistiquées (machines à commande numérique, informatique lourde, procédés automatisés) les postes équipement-machines, maintenance et fournitures représentent une part sensiblement plus élevée pouvant atteindre, voire dépasser les $40 \%$. Dans les formations tertiaires, les frais divers de formation sont très nettement inférieurs, et par conséquent la dépense totale de formation sensiblement moins élevée (de l'ordre de $30 \%$ ).

Tableau 4. Exemples de coût/apprenti (pour l'année 1990)

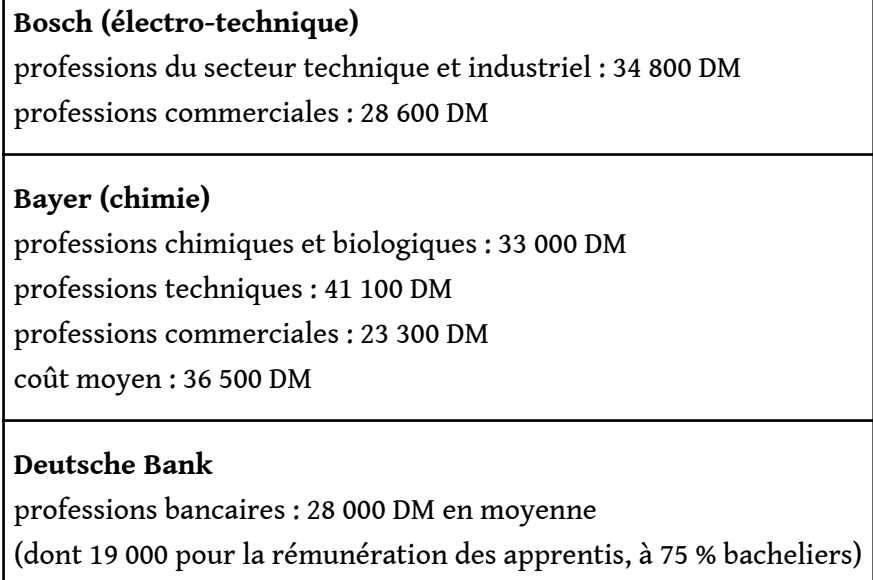

15 Selon l'importance numérique relative des apprentis (population des apprentis rapportée à l'effectif global), la part des dépenses de formation initiale dans la masse salariale varie entre 2,5 et $5 \%$. Ainsi chez Bayer AG (Allemagne), qui employait en 1990, 4103 apprentis sur un effectif de 63568 salariés (6,45\% de l'effectif), la part de la dépense de formation initiale dans les dépenses totales de personnel (pensions comprises) s'établissait à 2,8\%. Dans le cas de la Deutsche Bank, dont le "taux d'apprentissage" est un des plus élevés d'Allemagne ( $11 \%$ de l'effectif!), l'effort de formation initiale représente $5 \%$ des frais de personnel.

\section{La prestation productive de l'apprenti}

16 L'ensemble de ces coûts constitue ce que l'on appelle en Allemagne la dépense brute de formation dont il convient de défalquer la prestation productive réalisée par les apprentis au cours de leur formation, principalement dans la seconde période au cours 
de laquelle ils sont progressivement affectés à des postes de travail (généralement à partir de la troisième année dans les grandes entreprises industrielles, plus tôt, généralement dès la deuxième année, dans les PME, l'artisanat et les formations tertiaires).

Cette prestation productive est naturellement difficile à évaluer, car elle varie à la fois selon les formations et le profil des apprentis, selon les secteurs et selon la taille de l'entreprise. Elle est générée principalement par le travail effectif de l'apprenti lorsqu'il est affecté à un poste de travail. Elle ne peut cependant être calculée simplement à partir des heures de travail correspondantes, car pendant ces séquences il faut tenir compte du temps consacré au tutorat, à la fois par le tuteur et l'apprenti, et d'une productivité moindre de ce dernier.

Il n'en reste pas moins que dans les PME et les entreprises artisanales, où pour l'essentiel, une fois les techniques de base acquises au cours de la première année, l'apprentissage consiste à acquérir un savoir-faire "on the job", la prestation productive de l'apprenti est substantielle. Le fait que l'apprenti ait une productivité inférieure à celle d'un salarié normal est d'emblée compensé par sa rémunération moindre, si bien que l'on peut considérer qu'il est "moyennement" productif pour l'entreprise pendant tout le temps qu'il passe en poste de travail au cours des 2 dernières années et demie de sa formation (environ les $2 / 3$ de son temps de formation, puisqu'il continue à fréquenter l'école 1 jour et demi par semaine). L'apprenti est ainsi "moyennement" productif pendant 20 mois sur les 42 mois que dure sa formation.

Mais si l'on considère que, dans les 12 ou 15 derniers mois, il est "doublement" productif, puisqu'il est pour ainsi dire en mesure d'assurer les tâches d'un ouvrier qualifié alors qu'il perçoit moins d'un demi-salaire, on peut conclure que l'apport de l'apprenti au cours de sa dernière année et demie de formation compense entièrement, sinon plus, la charge intégrale qu'il représente pendant les 12 premiers mois de sa formation. De sorte que globalement, dans le cas des PME et de l'artisanat, on peut considérer que le coût net de l'apprenti est nul, voire négatif, ce qui signifie que la charge qu'il représente est entièrement compensée, sinon plus, par sa prestation productive.

Cela est dû pour l'essentiel au fait que la durée de formation est suffisamment longue pour pouvoir assurer et "amortir" l'investissement-formation, c'est-à-dire à la fois donner à l'apprenti une formation suffisamment solide et lui permettre de l'appliquer efficacement dans l'entreprise. On retrouve une "rentabilité" sensiblement analogue pour les formations commerciales ou tertiaires, dans lesquelles les frais fixes sont moins élevés et le passage de l'apprenti au poste de travail en situation opérationnelle est également relativement précoce (environ à mi-parcours du temps de formation, en moyenne).

21 La situation est moins favorable dans les grandes entreprises industrielles dans lesquelles l'encadrement est plus structuré, les frais fixes sont nettement plus élevés, et la formation technique est plus sophistiquée, et donc plus longue, avant que l'apprenti ne puisse être "lâché" en situation productive. Cela n'intervient généralement qu'au cours de la troisième année, sur une douzaine de mois effectifs tout au plus. Dans ce cas de figure, la prestation productive ne compense au mieux que 40 à $50 \%$, parfois moins, de la dépense brute de formation. 
Etant donné que les grandes entreprises forment un nombre élevé d'apprentis (souvent plusieurs milliers) à des coûts nets relativement élevés, elles se montrent de plus en plus attentives à l'efficacité de leur dépenses de formation initiale qui se chiffre souvent à plus d'une centaine de millions de DM (Bosch : 173 millions de DM, Bayer : 134 millions de DM en 1990). Aussi les dépenses des services de formation (initiale et continue) des grandes entreprises font-elles l'objet d'un contrôle de plus en plus strict. Ainsi le service de formation central de Bosch s'est-il vu étoffer d'un service de contrôle de gestion-formation chargé de veiller, dans l'ensemble du groupe, à une formation dispensée au meilleur coût. Signe évident, confirmé à plusieurs reprises par nos divers interlocuteurs, que, dans les grandes entreprises où la formation a bien souvent un caractère pilote, son coût atteint progressivement les limites de l'effort financier qu'il est possible de consentir dans un contexte de concurrence internationale de plus en plus vive.

23 En outre, le fait qu'une partie seulement des entreprises - en moyenne de 20 à $25 \%$ d'entre elles - soient habilitées à assurer la formation initiale, et ce pour l'ensemble de l'économie, est de plus en plus lourd à supporter pour les entreprises formatrices. Aussi réfléchit-on, dans les milieux patronaux, à un système de mutualisation des charges au sein des branches professionnelles dans lesquelles les entreprises formatrices bénéficieraient d'un fonds de soutien auquel cotiseraient les entreprises nonimpliquées dans la formation. Cette solution, qui existe déjà sous une certaine forme dans le bâtiment, est cependant âprement débattue. Nombre de responsables patronaux redoutent que cette forme de socialisation du financement de la formation initiale soit la porte ouverte à un financement public ou para-fiscal et incite les entreprises à se décharger progressivement de leur responsabilité formatrice.

De cette analyse des coûts dans le système allemand ressort cependant une conclusion essentielle : la formation en entreprise n'est pas, comme on le pense généralement en France, plus coûteuse parce qu'elle est plus longue. Bien au contraire : plus la période passée en entreprise est courte, moins la dépense est rentable, simplement parce que, d'un côté, les coûts fixes par apprenti, rapportés au temps de formation, sont proportionnellement plus élevés, et que, de l'autre côté, la formation est à la fois moins bonne et plus difficilement amortissable. En formation comme ailleurs, il n'y a pas d'investissement rentable "au rabais". Pour n'être pas uniquement une charge pour l'entreprise d'accueil, et pour être réellement formatrice pour le jeune en formation, l'alternance nécessite donc des séquences en entreprise suffisamment longues. En dessous d'un certain seuil, qu'en Allemagne on considère être de deux tiers temps sur au moins 2 ans (formule appliquée aux apprentis déjà bacheliers), l'alternance relève davantage de l'initiation à la vie professionnelle (qui n'est en soi pas inutile, mais reste finalement peu efficace professionnellement) que d'une véritable formation. C'est sans doute ce qui, du point de vue de l'entreprise, la fait davantage considérer en France comme une "prestation sociale" devant légitimement donner lieu à compensation par voie d'exonérations que comme un réel investissement, qu'il conviendrait à la fois d'amortir et de rentabiliser par une mise en œuvre efficace. 


\section{La rentabilité indirecte de l'investissement-formation initial}

Au delà de la démarche comptable, qui consiste à procéder au calcul du coût net de la formation, doit également entrer en ligne de compte une appréciation "économique" de l'investissement-formation incluant ses effets d'opportunité pour l'entreprise. Ceuxci sont évidemment plus difficilement mesurables, mais méritent cependant d'être mentionnés :

1. La rentabilité de l'investissement-formation est à calculer sur la durée de présence ultérieure dans l'entreprise des apprentis recrutés. En règle générale, les entreprises recrutent leurs apprentis diplômés dans plus de 75 à 80 \% des cas à l'issue de leur formation. Et, du fait de la d'intégration et de socialisation de l'apprentissage, les jeunes apprentis restent en général plusieurs années dans l'entreprise. Le taux de fidélité est naturellement variable selon les professions, selon les qualifications et les entreprises. La fluctuation est plus forte dans le tertiaire, notamment pour les bacheliers qui sont tentés de reprendre des études (ce qui ne signifie d'ailleurs pas qu'ils quitteront définitivement l'entreprise), ou de chercher à améliorer leur situation en étant mobiles. La fluctuation est également relativement plus élevée dans les PME industrielles. Elle est beaucoup plus faible dans les grandes entreprises où la durée moyenne d'emploi dans l'entreprise tourne autour de 10 à 12 années. Rapporté à une durée moyenne globale d'emploi que l'on peut estimer avec vraisemblance, d'après nos observations, à 5 ou 6 ans, le coût relativement élevé de la formation initiale se trouve ramené à quelques milliers de marks par an, ce qui représente finalement un coût minime pour des salariés généralement très bien qualifiés et qui, entre temps, continuent d'élargir leur expérience. Enfin, le coût que représente la formation initiale des apprentis exerce une "contrainte vertueuse" du point de vue de la politique de développement du personnel et de la gestion des compétences : pour fidéliser et motiver les jeunes salariés qu'elle a elle-même formés, bref pour rentabiliser son investissement formateur, l'entreprise doit s'efforcer de leur proposer un parcours professionnel qui permette de valoriser leurs compétences et leur assurer des voies de promotion (cf. chapitre $\mathrm{VI})$.

2. De ce coût d'amortissement de la formation initiale, il convient en outre de déduire le coût qu'aurait représenté le recrutement externe (petites annonces, procédure de recrutement) des jeunes salariés correspondants, puis celui de leur initiation-adaptation et éventuellement de la formation complémentaire de quelques mois qu'il aurait fallu leur dispenser avant qu'ils ne deviennent entièrement opérationnels dans l'entreprise.

3. En outre, une formation initiale qui assure une qualification technique de très bon niveau dispense l'entreprise de dépenses de formation continue à des fins de rattrapage ou de mise à niveau. Ces dépenses constituent en France, on le sait, une part non négligeable de l'effort de formation des entreprises qui doivent compenser, souvent dès le départ, les insuffisances de la formation scolaire. C'est sans doute ce qui explique qu'en règle générale, comparées aux dépenses de formation des entreprises françaises, celles des entreprises allemandes représentent un pourcentage moins élevé de la masse salariale : la différence est d'environ 1 à 2 points pour des entreprises de taille comparable. En Allemagne, la formation professionnelle continue peut, de ce fait, se concentrer sur sa fonction principale: le perfectionnement professionnel et l'acquisition de nouvelles compétences sur la base d'un socle solide de qualification préexistant.

4. La présence d'une main d'œuvre hautement qualifiée aussi bien au niveau de la production que du secteur administratif et commercial a, par ailleurs, des effets positifs sur la structure de commandement et le nombre des échelons hiérarchiques. Des salariés hautement qualifiés et opérationnels à tous les niveaux, et en particulier à celui des opérateurs, 
disposent d'une plus grande autonomie dans l'organisation et l'exécution de leur travail, ce qui permet de faire l'économie de fonctions d'encadrement et de contrôle à tous les échelons. De ce fait, la maîtrise et l'encadrement ont des effectifs moindres et sont davantage impliqués dans l'organisation de la production et le management proprement dits. C'est sans doute aussi ce qui explique le taux d'encadrement moindre et des échelons hiérarchiques moins nombreux constatés par tous les observateurs dans les entreprises allemandes, par comparaison avec les entreprises françaises ${ }^{2}$. L'investissement-formation initial contribue ainsi à une structure salariale plus productive, en permettant de faire l'économie d'échelons inutiles, qui sont non seulement coûteux en masse salariale, mais également facteurs de rigidité dans l'organisation.

5. La formation initiale est en outre un puissant facteur de production et de transmission de la culture technique de l'entreprise, principalement par l'intermédiaire des formateurs et tuteurs qui constituent en quelque sorte la mémoire de l'entreprise et la transmettent de génération en génération. Fonction irremplaçable que la formation continue à elle seule, et encore moins une politique de communication interne, aussi coûteuse et efficace soit-elle, ne sauraient réellement remplacer.

6. Enfin, du point de vue macro-économique et macro-social, le système de formation duale offre le meilleur rapport coût-efficacité :

- pour l'allocation des moyens de formation, en particulier pour les équipements techniques lourds nécessaires à la formation. Intégrés à l'entreprise, ils sont plus modernes et mieux adaptés, leur renouvellement est plus fréquent et à moindre coût unitaire pour la collectivité (les machines sont renouvelées avec le parc de l'entreprise, donc plus fréquemment à prix moindre que dans un établissement scolaire) ;

- pour l'insertion des jeunes : pour une dépense éducative globale à peu près comparable à celle de la France (RFA : $6 \%$ du PIB, France : 5,65\% du PIB en 1990) ${ }^{3}$ mais répartie différemment entre les collectivités publiques et les entreprises, le système allemand parvient à un taux de chômage des jeunes nettement inférieur. A la grande différence de la France où le taux de chômage des jeunes de moins de 25 ans est environ le double $\mathrm{du}$ taux de chômage moyen, en Allemagne celui-ci ne s'est que faiblement écarté $\mathrm{du}$ taux de chômage moyen tout au long des années 80 , et ce en dépit des variations conjoncturelles (cf. chapitre VI).

En outre, le système allemand conduit à une insertion professionnelle nettement plus précoce, puisque la presque totalité des apprentis, soit plus des deux tiers d'une classe d'âge, est employée à l'âge de 20 ans ; ce qui n'est pas sans conséquences du point de vue financier, puisque cela permet de faire l'économie d'un dispositif d'insertion professionnelle des jeunes de plus en plus complexe dont le coût énorme est en outre difficile à chiffrer exactement et dont l'efficacité demeure limitée. Cela représente également un avantage du point de vue du taux d'activité effectif, puisque cela permet non seulement d'utiliser au mieux les ressources de main-d'œuvre disponibles, mais fournit également une base de recettes plus large pour l'impôt et le financement de la protection sociale. 


\section{NOTES}

1. Cf. Bundesministerium für Bildung und Wissenschaft, Berufsbildungsbericht 1992, Bad Honnef, 1992, p. 160.

2. Cf. Burkhardt Lutz, "Bildungssystem und Beschäftigungsstruktur in Deutschland und Frankreich. Zum Einfluß des Bildungssystems auf die Gestaltung betrieblicher Arbeitskräftestrukturen", in : H. G. Mendius et al., Betrieb, Arbeitswelt, Qualifikation, Francfort s. M., 1976 et M. Maurice/F. Sellier/J.-J. Silvestre, Politique d'éducation et organisation industrielle en France et en Allemagne, Paris, PUF, 1982.

3. D'après la comparaison des chiffres fournis par le ministère de l'Education nationale, Repères et références statistiques sur les enseignements et la formation, éditions 1990 et 1991, et par le Bundesministerium für Bildung und Wissenschaft, Grund- und Strukturdaten 1990/91, Bad Honnef, 1990. 


\section{Le système dual entre l'école et la vie professionnelle}

1 L'analyse de la relation entre formation et emploi manifeste bien les avantages de l'apprentissage dual. En effet, elle montre d'une part que l'accès au système est largement ouvert, ce qui n'empêche pas des taux de succès élevés, d'autre part que la formation acquise est un atout précieux pour l'insertion professionnelle.

\section{Un accès ouvert et un faible degré d'exclusion}

2 Bien qu'étant une filière de masse - et même si l'observateur français, conditionné par les problèmes du collège unique devait s'en étonner - la formation duale ne connaît pas de problèmes majeurs d'échec.

Pour l'expliquer, il faut tout d'abord s'arrêter à l'articulation entre le système scolaire d'enseignement général et l'enseignement professionnel. La formation professionnelle ne peut en effet drainer la majorité d'une classe d'âge, comme nous l'avons montré au chapitre II, que parce que le système scolaire allemand est fortement différencié. L'orientation s'opère relativement tôt (au cours des $5^{\mathrm{e}}$ et $6^{\mathrm{e}}$ années de scolarité) vers 1 des 3 filières assez fortement étanches, la filière courte de la Haupt-schule ( 9 à 10 ans de scolarité), la filière moyenne de la Realschule (10 ans de scolarité, comportant des programmes plus riches et dont les enseignants sont mieux formés) et la filière longue du lycée Gymnasium (qui conduit au baccalauréat - Abitur). La Gesamtschule, école globale, introduite depuis le début des années 70 avec l'ambition de dépasser les cloisonnements du système traditionnel et d'éviter par une pédagogie différenciée la sélection précoce, n'a pas réussi à s'implanter et occupe aujourd'hui une position relativement marginale dans certains Länder. 
Figure 9. Le système d'enseignement en République fédérale d'Allemagne

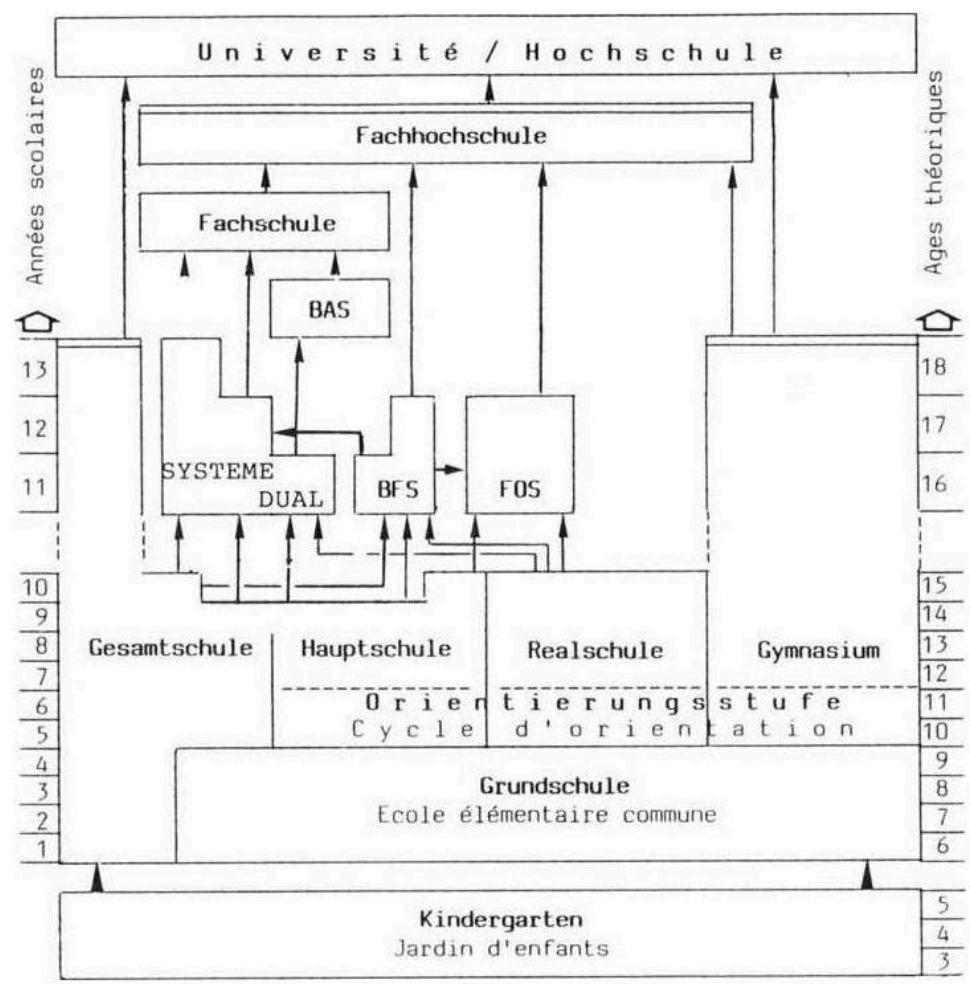

= Certificat d'accès à l'enseignement supérieur

BAS Berufsaufbauschule

BFS Berufsfachschule

BS Berufsschule

FOS Fachoberschule

4 En 1991, les effectifs de la $8^{\mathrm{e}}$ année de scolarité étaient pour $24,9 \%$ dans la Hauptschule, pour $31,5 \%$ dans la Realschule et pour $27,7 \%$ dans le lycée. C'est dire que $56,4 \%$ des élèves de ce niveau suivaient les filières courtes débouchant $a$ priori ${ }^{1}$ sur la formation professionnelle. Mesurée en pourcentage des classes d'âge correspondantes, les sorties des trois filières se répartissaient en 1991 comme suit : 40 \% des 15-16 ans venaient de la Hauptschule (dont $8,7 \%$, soit près de 54000 jeunes, sans avoir obtenu le certificat de fin d'études), $44 \%$ des 16-17 ans sortaient de la Realschule ou d'un cursus délivrant l'équivalent du certificat de fin de Realschule, tandis que $36,2 \%$ des 18 à 20 ans quittaient le système scolaire au niveau bac, $26,2 \%$ pour le bac général, 10,1 \% pour le certificat d'accès à la Fachhochschule ${ }^{2}$.

5 On lit en creux l'importance de la filière professionnelle pour les deux premières catégories, importance que confirme une coupe de la population résidente de 17 et 18 ans faite d'après les chiffres du micro-recensement de $1989^{3}$ Mis à part 3,7\% de chômeurs et $9,1 \%$ d'actifs, $44,5 \%$ des jeunes sont en formation professionnelle $(36,7 \%$ dans le système dual et $7,8 \%$ dans des écoles professionnelles à plein temps) tandis qu'ils ne sont que $37,7, \%$ en formation générale (dont $26,2 \%$ au lycée ${ }^{4}$.)

C'est dans cette articulation entre la formation générale et la formation professionnelle que réside la spécificité du système éducatif allemand: l'enseignement général peut paraître à l'observateur français particulièrement sélectif et élitiste, puisque, comme le montre le tableau 6 , la filière longue, peu ouverte comparativement à la France, recrute de façon socialement inégalitaire. En revanche, les deux filières courtes, qui 
rassemblent la majorité du public scolaire et dont les élèves viennent de milieux sociaux très variés (on remarquera en particulier la grande homogénéité de la distribution sociale dans la Realschule, qui mérite bien son nom d'école moyenne), ne sont évidemment pas perçues comme des voies de relégation; et, comme on le voit par ailleurs au tableau 5, la formation professionnelle sur laquelle elles débouchent, n'est nullement un ghetto ouvrier. Encore ces données sous-estiment-elles la part des enfants de familles aisées qui s'engagent dans la formation professionnelle, car un pourcentage notable de bacheliers, où ces catégories sont sur-représentées, font aussi ce choix.

7 On est donc à l'opposé de la logique qui est à l'œuvre en France, où, dans un contexte où les entreprises sont très peu impliquées dans la formation initiale, l'importance donnée à la démocratisation de l'accès aux cursus scolaires longs, qui structure les comportements éducatifs, finit par nuire à la promotion professionnelle et sociale qui en est attendue. Car indépendamment du fait que la démocratisation par l'école bute nécessairement sur la persistance des inégalités sociales, la course au diplôme d'enseignement général le plus élevé accroît le caractère sélectif des études. Or, la sélection dans le système de formation générale s'opère nécessairement sur des critères scolaires, donc étrangers aux compétences pratiques. Pourtant, c'est cette sélection qui décide de l'orientation vers les filières professionnelles. La conséquence est triple : d'une part, les jeunes qui se dirigent vers ces filières le font à l'issue d'un choix négatif, socialement peu valorisant et psychologiquement peu motivant; d'autre part, les formations qu'ils reçoivent ne sont pas suffisamment capables de transmettre le professionnalisme; enfin, ils connaissent une insertion professionnelle difficile et des possibilités de promotion plus réduites : le système attirant les meilleurs éléments vers les formations générales longues, ils subissent en effet la concurrence des diplômés issus de ces filières, auxquels les entreprises font plus volontiers appel, même pour les emplois de qualification moyenne. 
Tableau 5. Position des 17-18 ans vis-à-vis du système éducatif selon la profession du chef de famille en 1989

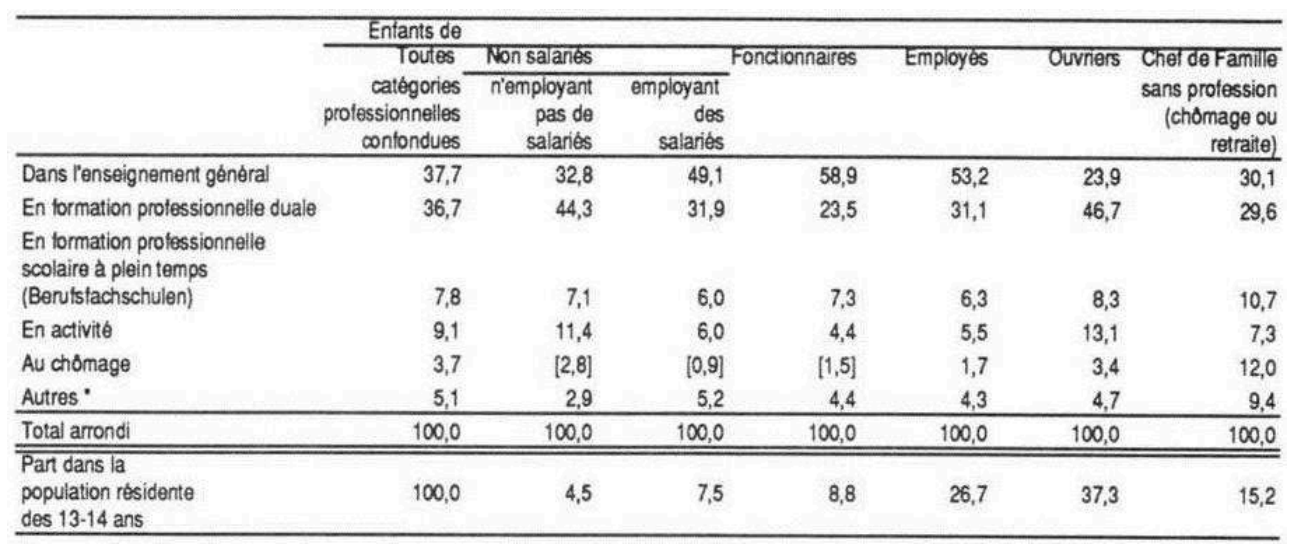

* sans réponse, études supérieures, Berufsschule seule

N.B : On notera le caractère peu différencié de la statistique professionnelle allemande, qui ne facilite pas l'interprétation. Ainsi, malgré la moins grande extension du statut de fonctionnaire qu'en France, il est clair que cette catégorie regroupe un large éventail de revenus et de positions sociales. II en va de même pour celle des non-salariés. Toutefois, la distinction entre les non-salariés employant ou n'employant pas eux-mêmes des salariés apporte un peu de clarté. Ainsi, compte tenu de

l'organisation du système de santé en RFA, où les cabinets médicaux fonctionnent dans la plupart des cas avec du personnel, les médecins appartiennent majoritairement à la première catégorie. La seconde inclut certes les actifs exerçant et des agriculteurs, donc de professions correspondant plutôt à un statut social et à un revenu modestes.

Source : Mikrozensus 1989

Tableau 6. Scolarisation des 13-14 ans selon la profession du chef de famille en 1989

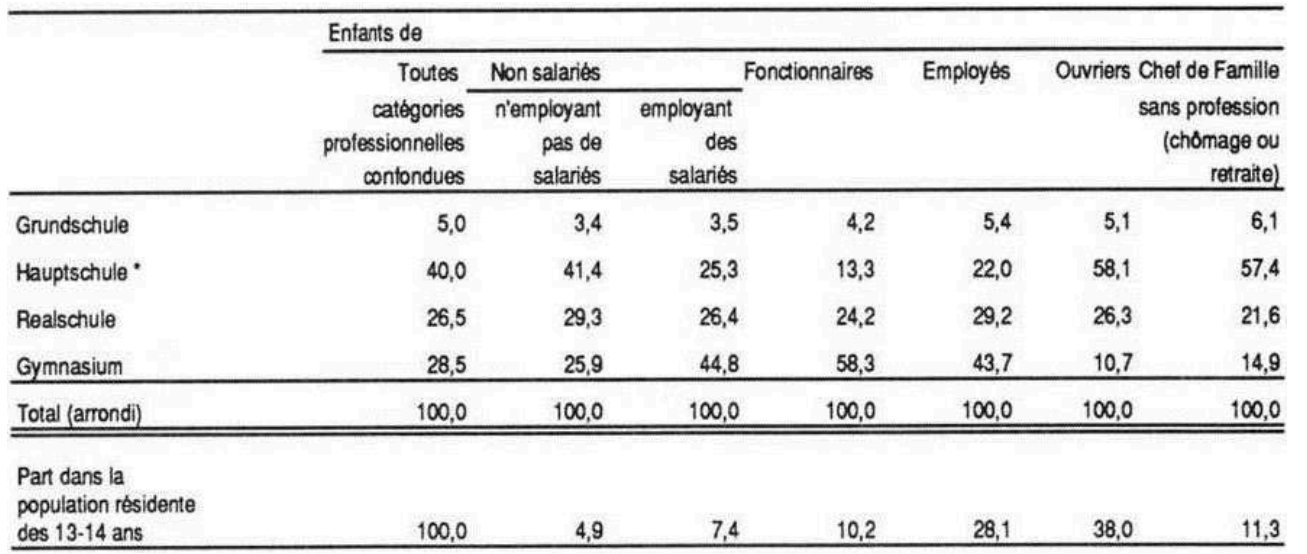

* et Sonderschulen, ce qui fausse un peu les chiffres.

N.B. : En Allemagne, on passe le baccalauréat à 18 ou 19 ans, donc à un âge postérieur à celui qu'analyse le tableau. Cf. aussi, dans partie, la répartition des apprentis en fonction des niveaux de formation générale.

Source : cf. tableau 5

8 En Allemagne, l'enseignement professionnel, élément central du système éducatif, a jusqu'alors peu subi la concurrence de la filière longue de l'enseignement général. Il puise donc dans un réservoir de capacités individuelles très différenciées et opère la sélection/orientation en fonction de critères cohérents avec son objectif de qualification pratique. Lieu d'acquisition d'une compétence reconnue et directement monnayable sur le marché du travail, sa forte légitimité ne s'explique pas seulement 
par une tradition de professionnalisme, mais surtout par les avantages qu'il procure en termes d'insertion et de promotion professionnelles.

9 Avant d'aborder ces deux derniers aspects en examinant, dans la deuxième partie de ce chapitre, les conditions de sortie de l'apprentissage, il faut insister sur les conditions d'entrée dans la formation duale. Celle-ci tire parti de l'hétérogénéité des recrutements : la grande diversité des entreprises impliquées dans la formation permet en effet une grande variété des niveaux d'apprentissage possibles selon les métiers concernés et en fonction des capacités technologiques et pédagogiques des unités formatrices. Et comme le dit Jean-Jacques Silvestre, c'est cette variété qui “donne un sens et un débouché à la diversité des niveaux sociaux et scolaires de ceux qui s'y engagent" 5 .

10 En pratique, la régulation de l'entrée en apprentissage obéit au mécanisme du marché. Les entreprises définissent librement leurs critères de sélection. Celle-ci s'opère la plupart du temps à partir du dossier scolaire, après passage de tests psychopédagogiques ou - dans certains métiers techniques - d'habilité manuelle ainsi que d'entretiens. Une partie des tests et entretiens sont faits de plus en plus souvent en groupe pour évaluer les qualités comportementales. Comme nous l'avons dit, cette sélection peut être plus ou moins rigoureuse selon les exigences du métier, mais aussi les besoins, les ambitions et les possibilités de formation de l'entreprise. Cette maîtrise des flux et de la qualité des apprentis conditionne fortement les taux de succès. Il est clair cependant qu'elle ne peut être totale, puisqu'il faut aussi tenir compte des conditions du marché, des caractéristiques structurelles du bassin d'emploi, de la situation conjoncturelle qui y règne, ainsi que de ce que font les concurrents, qui sont évidemment aussi sur le marché des apprentis potentiels. Naturellement, ce sont les entreprises réputées meilleures formatrices, attirant par conséquent les candidatures, qui sont le plus susceptibles de se soustraire à ces contraintes.

11 En 1991, 32,3 \% des apprentis venaient de la Hauptschule, dont 1,7 \% sans avoir obtenu le certificat de fin d'études. Cela signifie qu'environ 37000 jeunes en situation d'échec scolaire avaient pu accéder à une formation en entreprise, la plupart du temps dans l'artisanat. 35,5\% avaient un certificat de fin de Realschule (ou un équivalent), tandis que 10,4\% venaient d'une Berufsfachschule. La part des bacheliers s'élevait à 17,2\%: ce sont donc plus de 200000 jeunes de ce niveau scolaire ${ }^{6}$ qui entraient en apprentissage pour se former surtout à des professions commerciales dans le secteur des banques, de l'assurance, de l'hôtellerie, du tourisme, mais aussi à certaines fonctions spécialisées de l'industrie ${ }^{7}$.

12 Le fait que la moitié des bacheliers en apprentissage soient concentrés sur 10 professions, dans lesquelles, à une exception près, ils représentent entre un quart et plus de la moitié des effectifs d'apprentis, reflète bien les mécanismes de sélection qui opèrent à l'intérieur du système dual. Ceux-ci contribuent à expliquer le faible degré d'exclusion en cours de formation. Le taux de réussite aux examens, stable depuis longtemps, se situe en moyenne à $89-90 \%$, les chiffres les plus faibles oscillant autour de $75 \%$ dans certains métiers de l'artisanat (comme peintre ou électricien) ou dans des professions de bureau peu qualifiées (agent de bureau - Bürogehilfe), tandis que dans les services publics, les métiers commerciaux, mais aussi les métiers industriels les plus cotés, ils peuvent atteindre $95 \%^{8}$.

13 Les avantages pédagogiques inhérents au système dual sont un autre facteur de succès. La situation même d'alternance permet un lien particulièrement étroit entre théorie et 
pratique, quels que puissent être les problèmes de coordination qui se posent parfois entre entreprise et Berufsschule. Ce lien est d'autant plus fort que le va-et-vient entre théorie et apprentissage en situation professionnelle a lieu aussi à l'intérieur de l'entreprise, où est souvent également prodigué un enseignement théorique - à tel point que, en dépit du terme "dual", on peut dire que dans les grandes entreprises le système fonctionne en réalité sur trois pôles. Ce mode de formation a l'avantage de ne pas défavoriser les intelligences concrètes et inductives comme tend à faire l'enseignement purement scolaire. Elle est motivante dans la mesure où elle donne à voir rapidement le résultat et l'utilité de ce qui est appris. Enfin, elle responsabilise et socialise, facteurs également plutôt générateurs de succès.

Par ailleurs, il ne faut pas oublier l'effort pédagogique fait dans les entreprises. Les formateurs à titre principal sont tenus par la loi de passer un certificat d'aptitude pédagogique, qui suppose de suivre une formation correspondante. Si l'examen est normalisé, les formations des formateurs le sont peu ${ }^{9}$ ce que les pédagogues professionnels (tant les professeurs de Berufsschulen et que leurs formateurs à l'université) critiquent beaucoup. Il reste que, malgré la diversité des cas de figure, il y a dans beaucoup d'entreprises une forte sensibilisation à la dimension pédagogique et souvent une capacité d'innovation remarquable, que les organismes professionnels, tant patronaux que syndicaux, s'emploient à diffuser.

15 Ce bilan doit être cependant nuancé en évoquant le problème des interruptions d'apprentissage ${ }^{10}$. Alors que le taux des ruptures de contrat se situait aux alentours de $10 \%$ à la fin des années 70 , il a crû peu à peu dans la première moitié, puis plus brutalement dans la seconde moitié des années 80, pour dépasser les $20 \%$ depuis 1989 (23,2 \% en 1990).

Un quart seulement des apprentis en question quittent l'entreprise au cours de la période d'essai prévue à cet effet, la moitié interrompent leur formation au cours de la première année, le reste en deuxième ou troisième année. Bien que problématique pour les entreprises, le phénomène est moins inquiétant qu'on pourrait le croire tout d'abord, car si le taux de départ a fortement augmenté, la part des apprentis pour lesquels une rupture de contrat signifie l'abandon de toute formation professionnelle a baissé dans une proportion tout aussi forte ${ }^{11}$. Pour la majorité, il s'agit donc moins d'un échec que d'une mobilité au sein du système dual, ou d'une réorientation.

Pour comprendre l'ampleur prise par celle-ci, on avance trois sortes d'explication. La première s'attache aux changements de comportements et de mentalités liés euxmêmes à l'évolution économique et sociale : comme ils sont plus âgés ${ }^{12}$ les apprentis sont plus critiques vis-à-vis de la formation qui leur est dispensée ; comme ils ont plus de liberté, ils envisagent plus facilement d'allonger la phase de transition entre la sortie de l'école et le début de la vie professionnelle. La seconde explication souligne que ce type de comportement peut jouer parce que le marché des places d'apprentissage s'est détendu. Les jeunes, qui ont davantage le choix, peuvent se permettre d'être plus exigeants. De fait, on observe une plus forte différenciation des taux de rupture de contrat, d'une profession ou d'une entreprise à l'autre, selon la qualité de la formation proposée ${ }^{13}$. Les perspectives peu encourageantes sur le marché du travail, propres à faire douter d'avoir choisi le "bon" métier, ne peuvent que renforcer une telle attitude. Enfin, personne ne manque d'évoquer l'évolution vers une plus grande hiérarchisation des formations à l'intérieur du système éducatif au bénéfice des cursus longs. Formation courte, l'apprentissage dual devient moins attractif et doit donc recruter 
une proportion croissante d'apprentis sortant d'une carrière scolaire moins brillante, donc potentiellement plus instables. Les deux derniers arguments le montrent bien: l'augmentation des ruptures de contrat d'apprentissage est moins un problème en soi que le signe de difficultés plus fondamentales du système dual sur lesquelles nous reviendrons en conclusion.

\section{La formation duale : un atout pour l'insertion professionnelle}

La primauté du système dual dans le système éducatif, constatée précédemment, se traduit naturellement par une prépondérance dans la répartition des diplômes. Celle-ci apparaît dans toutes les statistiques, tant en termes de flux qu'en termes de stocks.

Concernant les flux, les statistiques sur les diplômes décernés, dont on dispose depuis le milieu des années 70, montrent que l'apprentissage dual a formé chaque année de 4 à 5 fois plus de jeunes que le secteur universitaire : en 1987, avaient été délivrés 680000 certificats de formation professionnelle, tandis que le nombre de diplômes d'enseignement supérieur n'était que de 139000 (les chiffres respectifs pour 1975 étaient de 460000 et 107000 ). Depuis 3 ans, la forte progression des cursus universitaires fait évoluer ce rapport à la baisse ${ }^{14}$.

Si l'on considère par ailleurs le stock de qualification à travers le niveau de diplôme dans l'ensemble de la population active occupée (ventilation selon le plus haut diplôme obtenu), on constate qu'en $198958 \%$ des Allemands avaient suivi (avec succès) une formation professionnelle "de base" (apprentissage dual ou formation de nature purement scolaire - Berufsfachschulen $)^{15} ; 8 \%$ avaient une formation de technicien moyen, niveau Fachschulabschluß, c'est-à-dire un diplôme d'école technique nonuniversitaire (y compris les diplômes de maitre artisan ou de maîtrise industrielle) préparé la plupart du temps en formation continue, donc par des salariés étant passés au préalable par l'apprentissage; enfin, $11 \%$ avaient un diplôme de l'enseignement supérieur, tandis que $23 \%$ seulement n'avaient pas de diplôme (beruflicher Abschlu $\beta)^{16}$. La comparaison avec les chiffres antérieurs (cf. tableau 7) montre par ailleurs que cette prépondérance des formations professionnelles de base n'a fait que s'affirmer depuis le milieu des années 70 .

Tableau 7. Population active occupée selon le diplôme le plus élevé obtenu

\begin{tabular}{|l|l|l|}
\hline & 1989 & 1976 \\
\hline Diplôme universitaire & $11 \%$ & $7,2 \%$ \\
\hline Formation niveau technicien (Fachschulabschlu $\beta$ ) & $8 \%$ & $6,4 \%$ \\
\hline Formation professionnelle (système dual et Berufsfachschule) & $58 \%$ & $52,1 \%$ \\
\hline Sans diplôme/Sans réponse & $23 \%$ & $34,3 \%$ \\
\hline
\end{tabular}

Source : Statistisches Bundesamt, Mikrozensus 1976 et Mikrozensus 1989. 
En Allemagne, les vingt dernières années n'ont donc pas été seulement une période d'expansion universitaire, puisque, entre 1976 et 1989, le pourcentage des actifs possédant un diplôme universitaire augmente de 3,8 points ; l'important est que, dans le même temps, la part des actifs ayant une formation professionnelle de base a gagné 6 points. En fait, comme les résultats en flux évoqués précédemment le suggèrent, le secteur universitaire et l'apprentissage ont connu une progression proportionnelle. C'est dire que la concurrence entre formations longues et formation professionnelle courte, dont il sera question dans le chapitre conclusif, n'est pas un phénomène sensible depuis longtemps. Comme les formations de technicien ont dans le même temps, elles aussi, progressé, on ne peut observer qu'une forte diminution de la part des actifs nonformés : supérieure à un tiers en 1976, elle représente en 1989 moins du quart de la population de référence ${ }^{17}$.

Si l'on en vient aux conséquences de cette prépondérance dans les niveaux de qualifications sur l'insertion professionnelle, les résultats du micro-recensement allemand de 1989 (cf. tableau 7) corroborent ce que les études des années 70 avaient déjà montré1 ${ }^{18}$ : il existe en Allemagne un lien très étroit entre la formation de l'individu et la qualification reconnue par l'employeur, alors qu'en France cette dernière dépend beaucoup plus de la formation sur le tas et de l'ancienneté. Ainsi, l'accès au statut d'ouvrier qualifié ou d'employé de niveau correspondant est, comme le montrent les tableaux, très largement conditionné par la possession d'un brevet dual. Inversement, les ouvriers n'en étant pas titulaires sont la plupart du temps classés non ou semiqualifiés.

Tableau 8. Niveau de diplôme des ouvriers par niveau de qualification

\begin{tabular}{lrrr}
$\begin{array}{l}\text { En Allemagne; 1989; hors apprentis; en \% } \\
\text { Niveau de diplome }\end{array}$ & $\begin{array}{c}\text { Non qualifiés ou } \\
\text { semi-qualities }\end{array}$ & Qualifiés & Ensemble \\
\hline $\begin{array}{l}\text { Hauptschule, Realschule, sans } \\
\text { formation professionnelle }\end{array}$ & 52,2 & 10,0 & 33,3 \\
$\begin{array}{l}\text { Brevet dual, ou certificat scolaire } \\
\text { equivalent }\end{array}$ & 44,4 & 84,8 & 62,6 \\
Bac seul & 1,2 & 0,3 & 0,8 \\
Bac + brevet dual & 0,7 & 1,9 & 1,2 \\
Brevet de technicien, de maltrise & 1,1 & 2,7 & 1,8 \\
Diplome supérieur & 0,4 & 0,3 & 0,3 \\
\hline Ensemble & 100,0 & 100,0 & 100,0 \\
En France; 1989; hors apprentis; en \% & & & Ensemble \\
Niveau de diplOme & Non qualifies & Qualifies & 59,5 \\
\hline CEP, BEPC, sans formation protessionnelle & 71,4 & 51,7 & 37,0 \\
CAP, BEP & 26,0 & 44,2 & 2,9 \\
Bacs, BT, BP & 2,1 & 3,4 & 0,6 \\
Diplöme supérieur & 0,5 & 0,7 & 100,0 \\
\hline Ensemble & 100,0 & 100,0 &
\end{tabular}

Source : Economie et statistique n² 246-247, sept.-oct. 1991, p. 84

Pour commenter cette relation qualification-emploi, il ne suffit pas d'évoquer une adaptation du système de formation professionnelle à l'appareil économique. D'abord, parce que ce serait ignorer une dimension sociale à laquelle nous avons déjà fait 
allusion en employant le terme de "culture professionnelle". Comme le dit J.-J. Silvestre, il faut parler "d'une identification du système de formation à la forme du salariat industriel qui existe en Allemagne". En effet, "la possession d'un [...] diplôme professionnel n'est pas uniquement un élément de qualification et de savoir-faire : c'est un élément de statut. Sa reconnaissance par les entreprises [...] n'est pas qu'une somme d'appréciations localisées et d'adaptation des formations aux emplois : c'est une norme sociale qui conditionne la forme d'existence de l'entreprise allemande".

De fait, et c'est le deuxième aspect qu'il faut souligner pour apprécier les avantages du système dual, celui-ci marque autant la "forme d'existence" de l'entreprise qu'il s'y adapte. Car, comme l'ont montré les travaux du LEST d'Aix-en-Provence et de l'ISF de Munich à partir d'une comparaison franco-allemande, le système éducatif n'est pas seulement le reflet des structures de l'emploi, mais agit également en retour sur celles$\mathrm{ci}^{19}$. Ainsi, lorsque les auteurs constatent dans les entreprises allemandes une division du travail verticale (nombre d'échelons hiérarchiques) et horizontale (séparation des fonctions) moins poussée qu'en France, une organisation qui y est moins bureaucratique et une définition des tâches qui donne davantage d'autonomie aux personnels qualifiés, ils y voient largement un effet des différences entre les systèmes éducatifs des deux pays.

A l'interface entre les contraintes techniques et l'offre de formation, l'organisation du travail peut profiter en Allemagne d'un personnel non seulement socialisé à l'entreprise, mais ayant acquis, grâce à l'apprentissage, des capacités professionnelles directement ou rapidement utilisables. Elle peut de ce fait être moins lourde et plus flexible qu'en France, où il faut davantage encadrer et former sur le tas. Cette flexibilité, qualité aujourd'hui particulièrement recherchée, explique évidemment le regain d'intérêt que suscite le système dual à l'étranger.

Une autre qualité essentielle du système dual est d'ouvrir des perspectives de promotion après les premières années de la carrière professionnelle. En Allemagne, il n'y a en effet pas de filière scolaire spécifique conduisant au niveau de technicien en formation initiale. La qualification requise par les emplois intermédiaires (entre ouvrier et ingénieur) s'acquiert donc sur la base du brevet dual, soit par promotion interne dans l'entreprise soit en suivant une formation continue dans une Fachschule après quelques années de pratique professionnelle.

Les analyses les plus récentes ont confirmé la stabilité des voies de recrutement à ces emplois en montrant que, depuis le milieu des années 70, la part des techniciens accédant à leur poste après un cursus universitaire ou de Fachhochschule est restée extrêmement minoritaire (moins de $10 \%$ ). Au sein de la voie promotionnelle, qui représente les $90 \%$ restants, la part des Fachschulen pendant cette période n'est, quant à elle, jamais inférieure à un tiers et a depuis quelques années tendance à s'élever. Il existe donc au sein des entreprises allemandes une logique de promotion ouvrière, qui profite doublement à la formation duale sur laquelle elle se construit: les entreprises peuvent gagner une certaine flexibilité dans la gestion de leur personnel grâce à l'apprentissage, puisque celui-ci peut alimenter à terme les échelons intermédiaires de la hiérarchie, et les jeunes, qui peuvent espérer pour leur part une promotion, sont, eux-mêmes, incités à choisir cette filière ${ }^{20}$.

La solidité des liens structurels entre formation et emploi, tels que nous les avons décrits, ne doit pas faire oublier l'existence de dysfonctionnements. Car le fait que la 
formation soit à l'initiative des entreprises ne garantit pas que leur offre corresponde exactement à la demande de l'appareil de production.

Indépendamment des déséquilibres conjoncturels que les employeurs peuvent difficilement anticiper, puisque la formation dure au moins 3 ans, le nombre de places de formation peut être volontairement supérieur aux besoins. C'est le cas dans un assez grand nombre de petites entreprises. Nous avons déjà relevé le phénomène en observant la fluctuation de la part de l'artisanat dans l'offre d'apprentissage en fonction de la situation du marché. Les apprentis, recrutés comme une main d'œuvre bon marché et n'étant pas embauchés en fin de formation, doivent non seulement trouver une autre entreprise, mais bien souvent aussi changer de métier, ce qui entraîne alors une déqualification. On sait que certains métiers de l'artisanat particulièrement concernés, comme celui de boucher ou de boulanger, constituent, en particulier dans les années de crise, un véritable réservoir de main d'œuvre pour l'industrie.

De manière plus générale, les parcours individuels d'insertion ne sont pas toujours aussi rectilignes que pourrait le suggérer notre analyse globale. Certes, les changements d'entreprise quelque temps après la fin de la formation ne doivent pas être a priori interprétés de façon négative. Ils sont pour une part volontaires et, dans la mesure où ils permettent une amélioration de la satisfaction professionnelle (meilleures conditions de travail ou perspectives de carrière), témoignent de la capacité des règlements d'apprentissage à standardiser les qualifications. Mais dans bien des cas, ils sont significatifs de problèmes ou au moins d'incertitudes.

C'est ce que montrent les études sur les trajectoires d'insertion, qui se sont multipliées depuis quelques années, en particulier depuis la pénurie de places d'apprentissage des années 80. A cet égard, le phénomène le plus frappant est le maintien des discontinuités bien qu'on soit entre temps passé à une situation d'excédent. Tout se passe comme si les comportements "d'esquive" (retraits passagers du marché, réorientation et cumul de formations, utilisation des possibilités de stages de formation complémentaire), explicables dans les années 80 par la difficulté d'accès au "droit chemin" de l'apprentissage dual étaient devenus structurels. De plus, l'activité dans les 3 premières années qui suivent la formation est marquée chez un nombre croissant de jeunes par des changements de profession, liés ou non à des périodes de formation continue ou des passages au chômage.

32 Pour expliquer le phénomène, on invoque à la fois les changements de mentalité chez les jeunes (individualisation, différenciation des conditions de vie - pour reprendre les thèmes sociologiques habituels ${ }^{21}$ ), et les mutations du marché du travail (incertitudes concernant les perspectives économiques et banalisation des emplois précaires, en particulier des contrats à durée déterminée pour les jeunes) ${ }^{22}$. L'Allemagne ne peut échapper ici aux évolutions qui touchent tous les pays industrialisés et qui, dans un contexte de flexibilisation générale des normes, brouillent peu à peu les contours du statut de travailleur qualifié.

33 Parallèlement, l'observation du chômage, pierre de touche de l'efficacité du système de formation professionnelle en termes d'insertion, pose elle aussi quelques problèmes. La difficulté principale tient à la structure complexe du marché du travail des jeunes. Il existe en effet ce que les Allemands appellent deux "seuils" (Schwellen) : le premier, situé entre l'école et le système dual, n'est qu'un marché des places d'apprentissage, 
alors que seul le second, au passage de la formation à la vie professionnelle, est un marché du travail au plein sens du terme.

L'observation du premier de ces deux marchés est particulièrement difficile, parce que tous les jeunes ne trouvant pas de place d'apprentissage ne sont pas enregistrés comme chômeurs : ils peuvent accepter une place dans un métier initialement non souhaité, quitte à changer ensuite d'orientation, s'engager dans une formation professionnelle scolaire pour attendre une situation meilleure ou continuer à chercher (en restant à la charge de leur famille) sans pour autant le signaler à l'office du travail. C'est pourquoi un déficit d'offre sur le marché des places d'apprentissage ne se traduit pas forcément par une hausse proportionnelle du taux de chômage des moins de 20 ans. En outre, il peut aussi, par un phénomène de vases communicants dû à la désorganisation des parcours de formation, rejaillir sur le taux de chômage des 20-24 ans. Si l'on ajoute les problèmes de convention statistique ${ }^{23}$ et les difficultés d'interprétation dues aux fluctuations démographiques, il est clair que les chiffres doivent être interprétés avec prudence.

Figure 10. Taux de chômage des groupes dits "à problèmes"

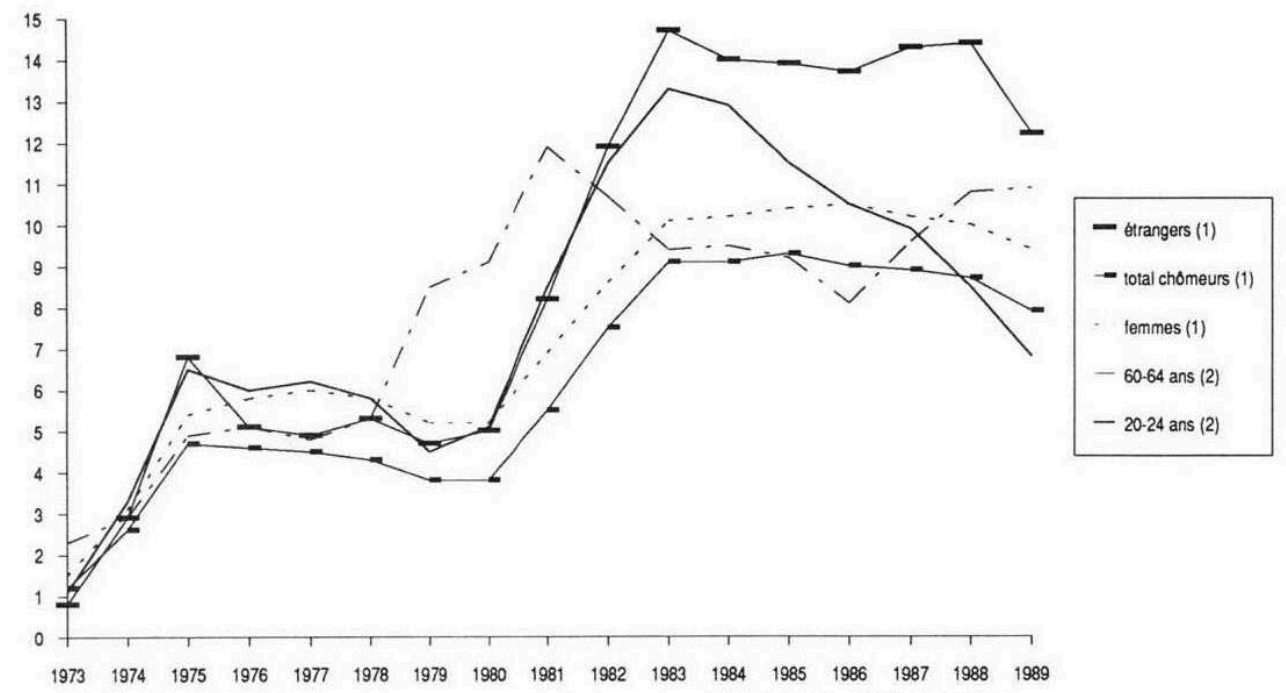

Source : Bundesanstalt für Arbeit

(1) moyenne/an (2) fin septembre 
Figure 11. Taux de chômage (1) en fonction des niveaux de diplôme (1975-1991) (2)

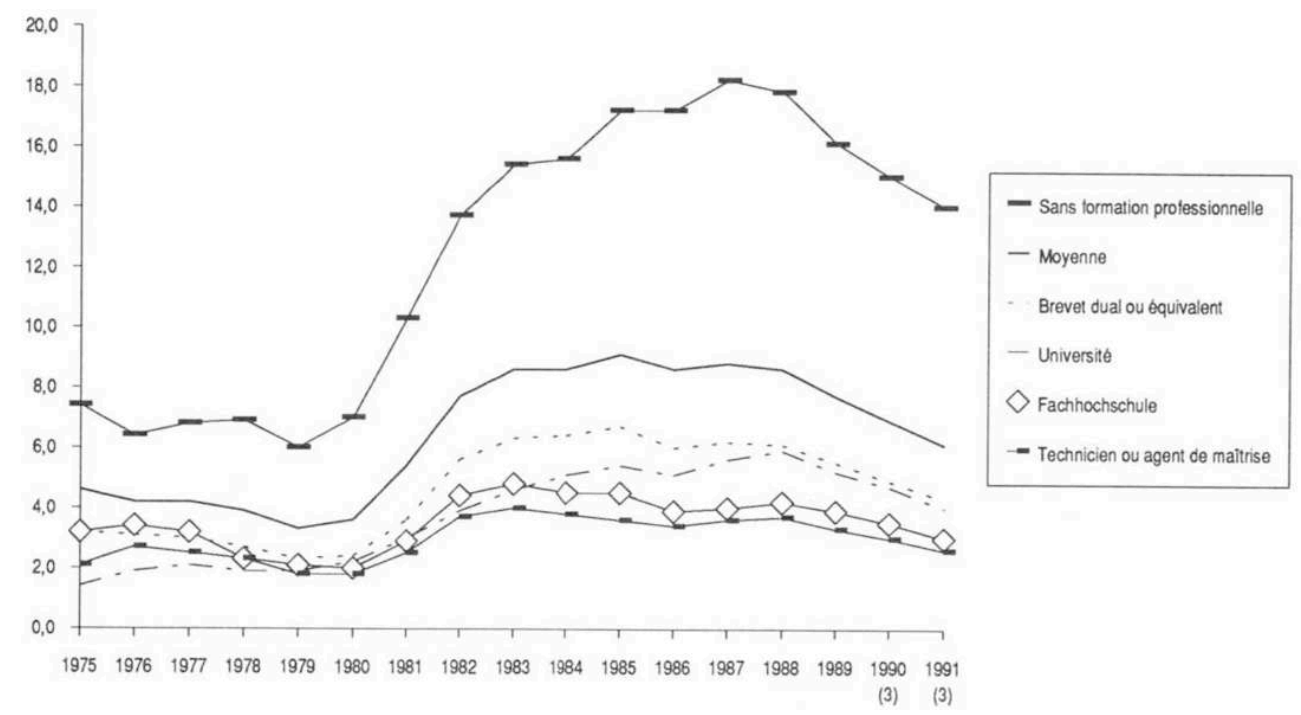

(1) en \% des actifs salairés à qualification équivalente, non compris soldats, appelés et apprentis

(2) fin septembre ; (3) données provisoires

Source : M. Tessaring (IAB), 1992, données non publiées

Malgré tout, les deux séries longues présentées ici (cf. figure 10 et figure 11) restent très parlantes. L'observation des groupes dits "à problèmes" (Problemgruppen) montre que le chômage des jeunes a été supérieur au chômage moyen dans les années de crise (il est aujourd'hui au même niveau que celui-ci). Mais bien que ces années aient vu l'arrivée sur le marché du travail de classes d'âge très nombreuses, au plus fort de la crise conjoncturelle, cet écart n'a jamais dépassé les $5 \%$. Il n'a donc aucune commune mesure avec l'écart que l'on connaît en France (autour de $15 \%$ dans la période considérée). Quant à l'évolution des taux de chômage en fonction des niveaux de diplômes, elle est encore plus probante : établie sur l'ensemble des chômeurs, donc tous âges confondus, cette statistique montre que la formation professionnelle a constamment minimisé le risque de chômage. Indépendamment de l'allongement des phases d'insertion, le système dual continue donc d'être un facteur puissant d'intégration au monde professionnel.

\section{NOTES}

1. "A priori”, car les jeunes peuvent, en empruntant les diverses passerelles décrites au chapitre II, réintégrer l'enseignement général. Les chiffres cités ici, comme ceux qui suivent, proviennent, sauf indication contraire, de Bundesministerium für Bildung und Wissenschaft, Grund- und Strukturdaten Bad Honnef, 1992.

2. Les pourcentages sont calculés par rapport à la moyenne des deux classes d'âge, et en tenant compte respectivement du nombre de jeunes jusqu'à 16, 17 et 19 ans révolus.

3. Les écarts avec les chiffres émanant de la statistique scolaire citée précédemment tiennent à la méthode employée (il s'agit ici de sondages), à l'année d'observation, 1989 au lieu de 1991 (à cet 
égard, les différences manifestent aussi l'attraction croissante qu'exercent les parcours scolaires longs, phénomène dont nous analysons les conséquences au dernier chapitre), enfin et surtout au fait que la catégorie des élèves de Hauptschule inclut aussi le public des écoles spéciales (Sonderschulen), destinées aux handicapés et aux cas sociaux.

4. 6,9\% sont à la Realschule, 3,3\% à la Hauptschule et $1,3 \%$ à la Gesamtschule. Si le total de ces chiffres ne représente pas $100 \%$, c'est qu'il faut y ajouter 5,1\% d'indéterminés, pourcentage qui regroupe les sans réponses, les élèves de Berufsschulen ne suivant pas de formation, mais aussi la frange de jeunes particulièrement doués, qui sont déjà à l'université ou dans une Fachhochschule. Les chiffres reprennent des données fournies dans Wolfgang Böttcher, "Soziale Auslese im Bildungswesen", in : Die deutsche Schule 2/1991.

5. J.-J. Silvestre, “La professionnalisation : l'exemple allemand", in : Pouvoirs 30, 1984.

6. Chiffres in: Grund- und Strukturdaten 1992/93, op. cit. Les valeurs absolues sont en revanche tirées de Bundesministerium für Bildung und Wissenschaft, Berufsbildungsbericht 1992, Bad Honnef, 1992, p. 30. La référence est, dans les deux cas, le nombre total de jeunes en apprentissage, il s'agit donc de chiffres en termes de stocks.

7. Cf. Berufsbildungsbericht 1992, op. cit., p. 30.

8. Idem, p. 41

9. Bien que la loi de 1969 ait créé le certificat de formateur en entreprise, les pouvoirs publics ne se sont pas préoccupés d'organiser (ou de normaliser) des formations correspondantes. Si bien qu'à la différence de la "pédagogie professionnelle" (Wirtschaftspädagogik), qui s'est développée à partir des années 20 après la remise en ordre des Berufsschulen et qui est aujourd'hui une matière universitaire à part entière, la pédagogie d'entreprise (Betriebspädagogik) n'a ni statut scientifique reconnu (ce qui en Allemagne est très important), ni institution scolaire spécifique. Les futurs formateurs en entreprise suivent des séminaires de formation continue qui sont la plupart du temps organisés par les chambres de commerce ou les organismes professionnels.

10. Sur tout ce qui suit, cf. Berufsbildungsbericht 1991, op. cit. et Heinrich Althoff, "Die fortdauernde Zunahme vorzeitiger Vertragslösungen und Erklärungsmöglichkeiten für diese Entwicklung", in : Berufsbildung in Wissenschaft und Praxis 4/1991, ainsi que Berufsbildungsbericht 1992, op. cit., p. 36 sq. et p. $112 \mathrm{sq}$.

11. A la fin des années 70 , c'était le cas pour $1 / 3$ des ruptures de contrat, aujourd'hui, seulement pour $1 / 3$.

12. L'augmentation de l'âge moyen des apprentis (16,6 ans en 1970, 18,8 en 1989 - en 1970, 22 \% avaient 18 ans et plus, aujourd'hui, c'est le cas de $72 \%$ ) peut s'expliquer par la demande, venant des élèves, d'une formation générale plus longue, par la demande, émanant cette fois d'une partie des entreprises, d'une préqualification générale plus élevée, par l'augmentation de la part des formations duales durant 3 ans ou 3 ans et demi, enfin par l'arrivée sur le marché de jeunes s'étant engagés dans des formations préprofessionnelles scolaires au moment de la pénurie de places d'apprentissage et souhaitant accéder enfin à l'apprentissage dual de leur choix.

13. Il n'est pas fortuit p. ex. que ces taux soient passés entre 1978 et 1989 de $15 \%$ à $31,7 \%$ pour l'artisanat (où les meilleures formations côtoient les pires) alors que, dans l'industrie et le commerce, ils ne sont passés que de 10,3 à 15,8\%.

14. En 1990, les chiffres respectifs étaient de 531600 et 148 200, soit un rapport de 3,5 à 1 . On compare ici le nombre des examens terminaux réussis chaque année, d'une part au terme de la formation professionnelle, d'autre part à la fin de tous les cursus de l'enseignement supérieur, universités, Fachhochschulen, etc. Il n'est évidemment pas exclu qu'un jeune ayant obtenu le certificat d'apprentissage poursuive des études pour se retrouver quelques années après dans la statistique des diplômés du supérieur. Les chiffres sont issus de Grund- und Strukturdaten 1992/93, op. cit.

15. Concernant les certificats du système dual et des cursus de Berufsfachschulen, nous employons le terme de "formation professionnelle de base" pour éviter de parler de niveau "ouvrier" 
professionnel. Ces formations concernent en effet autant le tertiaire ou les services publics que l'agriculture ou l'industrie et conduisent d'ailleurs à l'intérieur de cette dernière autant à des professions commerciales qu'à des qualifications ouvrières.

16. La classification allemande est en l'occurrence significative de l'architecture du système éducatif. Tous les diplômes cités, qu'il s'agisse des diplômes universitaires, des diplômes de technicien ou des diplômes de formation professionnelle de base, sont mis sur le même plan et qualifiés de beruflicher Abschluß. Les chiffres cités ici proviennent du Statistisches Bundesamt, Mikrozensus 1989, tels qu'ils sont reproduits par le Erich Schmidt Verlag (fiche 247154 2/91).

17. Chiffres du Statistisches Bundesamt reproduits dans iwd (Institut der deutschen Wirtschaft). Pour de très intéressantes comparaisons franco-allemandes, cf. Martine Möbus/Patrick Sevestre, "Formation professionnelle et emploi : un lien plus marqué en Allemagne", in: Economie et statistique $\mathrm{n}^{\circ}$ 246-247, sept.-oct. 1991. Alors que les titulaires d'un brevet dual ou d'un diplôme d'école professionnelle à plein temps représentent selon ces données $55,4 \%$ de la population active occupée allemande en 1989, les titulaires français d'un CAP ou d'un BEP ne représentent que 28,9\%. Les chiffres produits par Burkhardt Lutz à partir de données de 1970/71 montrent une différence plus accusée encore : 55,7 \% contre 20,3 \% (Burkhardt Lutz: "Bildungssystem und Beschaftigungsstruktur in Deutschland und Frankreich. Zum Einfluss des Bildungssystems auf die Gestaltung betrieblicher Arbeitskraftestrukturen", in: Hans-Georg Mendius et al., Betrieb, Arbeitsmarkt, Qualifikation, Francfort s. M./New York, 1976).

18. Cf. l'étude conjointe de l'ISF de Munich et du LEST d'Aix-en-Provence, B. Lutz, "Bildungssystem...", op. cit., et Marc Maurice/François Sellier/Jean-Jacques Silvestre, Politique d'éducation et organisation industrielle en France et en Allemagne, Paris, PUF, 1982.

19. Cf. B. Lutz, "Bildungssystem..., op. cit. 1976 et M. Maurice/F. Sellier/J.-J. Silvestre, Politique d'éducation..., op. cit.. La présentation la plus synthétique est due à Burkhardt Lutz, "Die Verantwortung der Bildungspolitik und der sogenannte Bedarf des Beschäftigungssystems einige Fakten und kritische Überlegungen“, in: Wilfried Schlaffke/Rainer Zelder (dir.), Die Zukunft der Berufsausbildung, Cologne, 1977.

20. Cette analyse reprend les conclusions de Ingrid Drexel/Philippe Mehaud, "L'accès aux emplois de technicien : voie scolaire ou voie promotionnelle ? Une comparaison France-RFA", in : Marine Möbus/Eric Verdier (dir.), Le système de formation professionnelle en RFA. Résultats de recherches françaises et allemandes, Paris (CEREQ, Collection des études n 61), février 1992.

21. Cf. par exemple le "classique" Ulrich Beck, Risikogesellschaft. Auf dem Weg in eine andere Moderne, Francfort s. M., 1986.

22. Sur ces questions, cf. Berufsbildungsbericht 1992, op. cit., p. 61 sq., qui cite les conclusions d'un certain nombre d'études récentes sur les parcours d'insertion. Cf. aussi Erich Raab, "Berufsausbildung und Arbeitsmarktchancen Jugendlicher in den alten Bundesländern", in : Aus Politik und Zeitgeschichte, 11/09/92 et surtout Gisela Westhoff/Axel Bolder (dir.), Entwarnung an der zweiten Schwelle ? Übergänge von der Berufsausbildung ins Erwerbsleben, Berlin/Bonn, 1991.

23. Ainsi, les séries statistiques détaillées permettant de ventiler le chômage par âge et par niveau de qualification sont seulement établies deux fois par an, en mai et en septembre. Comparer le chômage des jeunes avec le chômage de l'ensemble des classes d'âge actives, qui est établi en moyenne annuelle, n'est donc pas rigoureux. Mais comparer les valeurs de septembre pour lesquelles on dispose des séries longues les plus complètes - conduit également à des distorsions, car, compte tenu des variations saisonnières, le chômage global y est plutôt au plus bas, tandis que, par suite des calendriers scolaires, le chômage des jeunes (en particulier de moins de 20 ans) est supérieur à sa moyenne annuelle. Par ailleurs, le calcul habituel du taux de chômage, qui rapporte le nombre de chômeurs à la population active totale, peut biaiser les comparaisons France/Allemagne : les jeunes en formation ne se retrouvent pas dans le chiffre du dénominateur en France, alors qu'ils sont décomptés comme actifs en RFA. Cf. à ce sujet Gero Lenhardt/Karen Schober, "Berufliche Bildung und Arbeitsmarkt. Der schwierige Berufsstart: 
Jugendarbeitslosigkeit und Lehrstellenmarkt”, in: Max-Planck-Institut für Bildungsforschung Projektgruppe Bildungsbericht (éd.), Bildung in der Bundesrepublik Deutschland, Daten und Analysen 2, Reinbeck bei Hamburg, 1980, p. 939 sq. 


\section{Bilan et perspectives}

\section{Les vertus de l'alternance}

$1 \mathrm{Au}$ terme de cette étude, une constatation s'impose, c'est la grande capacité d'adaptation du modèle allemand d'apprentissage ${ }^{1}$. Celle-ci est évidente sur le long terme si l'on n'oublie pas que le terme "système dual"2 légitime si l'on s'attache à la continuité institutionnelle, recouvre en fait plusieurs types de formation professionnelle qui se succèdent en l'espace de 100 ans. Du modèle initial issu de l'idéal artisanal, plutôt archaïque au regard des réalités de la grande entreprise, on passe progressivement à partir des années 30 à la formation spécifique d'un travailleur de type nouveau, l'ouvrier professionnel d'industrie. Puis, après la consolidation légale de 1969 et une période de remise en cause radicale dans les années 70, le système connaît une nouvelle mutation, qui lui permet de retrouver sa pleine légitimité en intégrant l'effet des changements technologiques ${ }^{3}$.

2 Mis à part le dualisme institutionnel, l'autre élément de continuité dans cette longue histoire est ainsi le ressort de la modernisation réussie : c'est l'implantation au cœur de la sphère productive, qui permet à l'apprentissage de bien appréhender les besoins de qualification auxquels il se donne pour fonction première de répondre.

Ce diagnostic est confirmé par l'importance qu'a eue dans l'évolution récente du système la discussion sur l'évolution des modes de production ${ }^{4}$. Les années 70 avaient été dominées par un regard plutôt pessimiste sur les perspectives de l'organisation du travail. Le discours sociologique dominant ${ }^{5}$, considérant que le processus de rationalisation industrielle allait provoquer une polarisation croissante des qualifications, encourageait en effet une réforme du système éducatif allant vers un tronc commun jusqu'au bac qui intègre la formation professionnelle. Compte tenu de l'importance du phénomène de déqualification attendu, l'accès généralisé à une formation scolaire longue trouvait sa justification pour la gauche et les syndicats dans l'objectif d'une plus grande égalité des chances, ainsi que d'un développement des facultés critiques et de l'épanouissement personnel qui ne paraissaient guère favorisés dans le travail. Les vertus d'une formation intégrée à la production passaient nettement à l'arrière-plan. 
Depuis le milieu des années 80 , on assiste à un retournement de tendance. Un grand nombre d'analystes affirment que l'évolution des technologies crée les conditions d'une organisation du travail plus valorisante. Les nouveaux modes de production ${ }^{6}$ peuvent faire régresser le taylorisme et la centralisation, en permettant par exemple le travail en petits groupes au sein desquels se réaliserait une "reprofessionnalisation", notamment par la réintégration aux tâches de production de certaines tâches de contrôle et d'entretien. Dès lors que patrons et syndicats misent de concert sur un tel scénario, l'apprentissage dual, système cogéré, retrouve une forte légitimité : tel est l'acquis des nouveaux règlements de formation, qui ne peuvent être centrés sur la polyvalence que parce que chaque partenaire y voit la chance de profiter au mieux des potentialités technologiques nouvelles ${ }^{7}$.

5 Avec l'effacement de la perspective des années 70, qui prenait pour seul critère la démocratisation du système éducatif, l'apprentissage dual peut de nouveau faire valoir ses qualités intrinsèques ${ }^{8}$ :

1. il assure à l'économie une offre abondante de main d'œuvre hautement qualifiée ;

2. il contribue à maintenir à un niveau très bas le chômage des jeunes en les formant à des professions reconnues par les employeurs, puisque codéfinies par les entreprises et effectuées en leur sein;

3. il participe, compte tenu d'un taux d'apprentissage qui, rapporté à une génération, approche les $70 \%$, à l'intégration sociale de la majorité des jeunes en leur offrant une formation et des perspectives d'emploi ;

4.il remplit une fonction de socialisation en procurant aux apprentis les premières expériences de l'entreprise et de la vie professionnelle ;

5. enfin, il forme une main d'œuvre professionnelle au sein de laquelle, par le jeu de la mobilité interne, peuvent être recrutés un grand nombre de cadres moyens et même supérieurs ${ }^{9}$.

\section{Le tribut de la modernisation}

6 L'efficacité reconnue du système dual ne doit cependant pas faire oublier une série de problèmes. Les premiers sont d'ordre qualitatif et représentent en quelque sorte le tribut de la modernisation.

7 Comme nous l'avons dit au chapitre IV, l'importance désormais donnée aux qualifications-clés exige des entreprises un effort pédagogique et d'organisation, quand elle ne leur demande pas de former à des domaines techniques ne correspondant pas à leur équipement productif. Si une telle contrainte peut être maîtrisée par les grandes sociétés, qui ont des ateliers d'apprentissage, elle risque de mettre en difficulté beaucoup de PME. Celles-ci jouent pourtant un rôle stratégique, puisqu'elles offrent environ $2 / 3$ des contrats de formation. Dans ces conditions, les centres d'apprentissage inter-entreprises sont de plus en plus nécessaires. Si l'on considère cependant que les quelques 600 centres actuellement en service - pour la plupart dans l'artisanat occasionnent déjà des frais courants de plus de 800 millions de mark par an, il apparaît que la généralisation d'une telle solution risque de poser des problèmes financiers. Pourra-t-on en rester aux procédures de financement internes à l'économie (collecte de contributions par les chambres de commerce ou par des organismes de branche) ? Ou le débat sur un cofinancement public ne va-t-il pas bientôt ressurgir, avec ses suspicions et ses polémiques sur le risque d'étatisation du système ? $^{10}$. 
8 L'évolution qualitative du système a par ailleurs affecté le rôle de l'école professionnelle. L'objectif des nouveaux règlements de formation est en effet de transmettre aux jeunes non seulement des savoirs et des savoir-faire, mais de les rendre "capables de prendre des initiatives" (Handlungskompetenz) à l'intérieur de leur domaine technique. Les méthodes pédagogiques qui correspondent à cette ambition tendent donc à faire éclater les frontières tant disciplinaires que fonctionnelles, notamment en structurant l'enseignement autour de projets. C'est dire que la division du travail traditionnelle, déjà peu adaptée, qui confie la théorie à l'école et la pratique à l'entreprise n'a plus guère de sens.

9 Pour répondre aux nouveaux besoins, il faudrait non seulement que les Berufsschulen coopèrent étroitement avec les entreprises, mais aussi qu'elles rénovent et restructurent leur propre enseignement. Or, l'école professionnelle est en crise, car elle a trop peu de crédits, manque d'enseignants (faute de postes, mais aussi de candidats à ces postes), qui ont en outre trop peu d'occasions de se recycler. Sa position est donc faible, en particulier face aux grandes entreprises, qui organisent de plus en plus un enseignement théorique et professionnalisent leur pédagogie. Pour certains analystes, le déséquilibre est déjà si fort qu'on peut craindre une privatisation rampante de l'apprentissage ${ }^{11}$.

\section{Les problèmes de recrutement}

10 Mais les difficultés les plus préoccupantes pour l'avenir du système dual se manifestent de façon quantitative. L'évolution démographique et la modification des comportements éducatifs risquent en effet de poser à terme de sérieux problèmes de recrutement. Non seulement le nombre de jeunes en âge de faire un apprentissage diminue notablement par suite de l'arrivée des classes creuses, mais l'apprentissage dual voit sa prédominance dans le système éducatif allemand peu à peu grignotée, alors même que les scénarios d'évolution des structures de production ne laissent pas présager une diminution des besoins dans les types de qualifications qu'il délivre.

11 A cet égard, le fait que les effectifs universitaires aient, pour la première fois dans l'histoire éducative de l'Allemagne, dépassé en 1990 ceux de l'apprentissage a joué le rôle de révélateur dans l'opinion. La statistique, largement reproduite par la presse, est certes trompeuse compte tenu de l'inégale durée des formations, 3 ans et demi pour la formation duale, 6 ans en moyenne pour les études universitaires. Mesuré en flux d'entrée, le nombre de nouveaux apprentis reste aujourd'hui encore plus de deux fois supérieur à celui des étudiants de première année. Mais dix ans plus tôt, il y avait deux fois moins d'universitaires que d'apprentis.

12 C'est dire que le phénomène d'allongement des études n'est pas nouveau non plus en Allemagne, mais ayant commencé plus tard qu'en France et s'étant tout d'abord opéré sans diminuer la base de recrutement du système dual, il était jusqu'alors moins visible. Comme le montre la figure 12, de 1960 à 1986, le système dual a presque maintenu sa part dans les classes d'âge de 15 à 19 ans. Au vu des chiffres, cette stabilité semble s'expliquer par un effet de substitution : l'apprentissage a pu puiser dans le réservoir des jeunes qui auraient auparavant exercé une activité sans formation préalable pour compenser les départs en direction du système scolaire. Pour schématique que soit cette interprétation, elle situe bien le problème actuel: au fur et à mesure que le 
système scolaire attire davantage de jeunes, le "vivier" des apprentis potentiels ne peut que s'amenuiser. Le système dual semble donc arrivé à un tournant de son évolution.

Figure 12. Position vis-à-vis du système éducatif des 15-19 ans - en \% (population résidente)

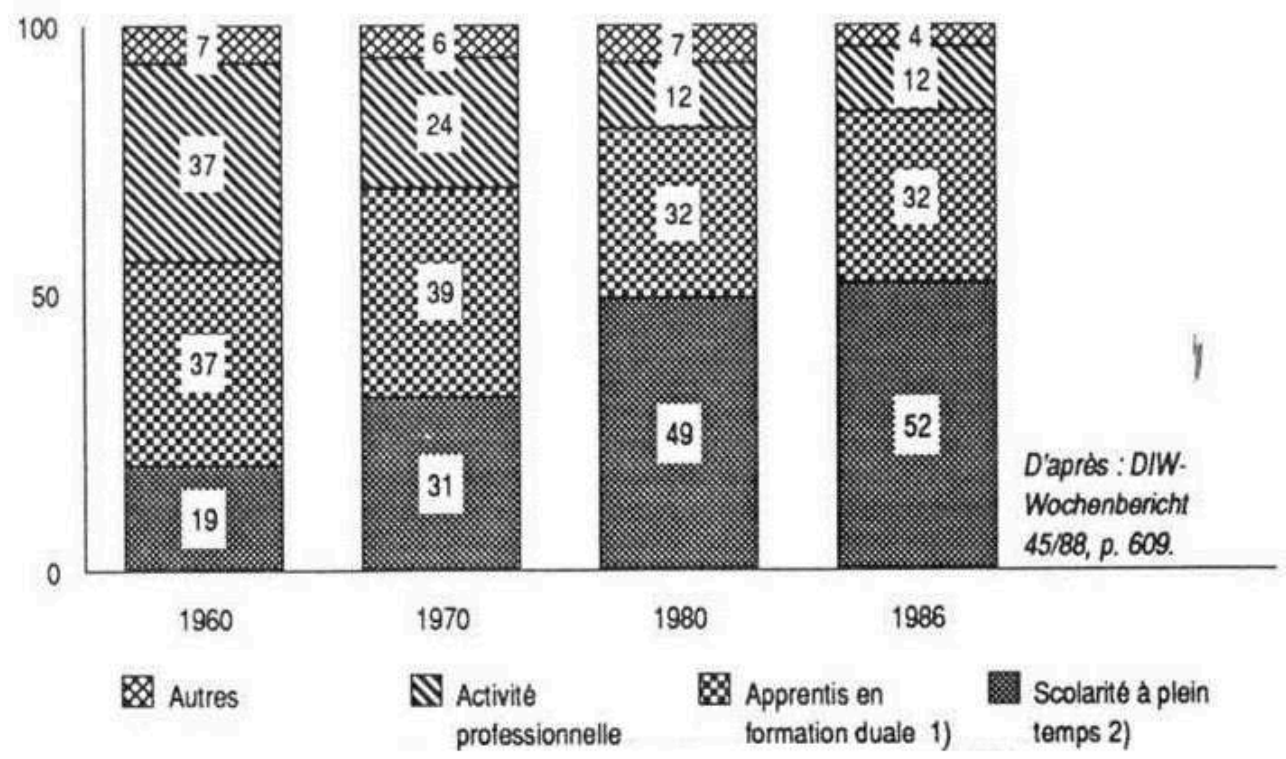

(1) Y compris les jeunes en activité ou au chômage de moins de 18 ans, qui sont tenus d'aller à mitemps à la Berufsschule. (2) Enseignement général, enseignement supérieur, écoles professionnelles à plein temps.

En pratique, ce phénomène se traduit déjà par un manque de main d'œuvre qualifiée. Selon le dernier rapport sur la formation professionnelle du gouvernement fédéral, l'offre de places d'apprentissage dual dépassait de plus de $20 \%$ la demande. Il est vrai que les données sur l'offre et la demande d'apprentissage publié par l'office fédéral du travail dramatisent la situation dans la mesure où, dans une telle conjoncture, un nombre croissant de jeunes trouvent une place d'apprentissage sans passer par les bureaux de placement officiels, mais le solde déficitaire n'est pas contestable : pour l'année 1991, il s'élève à environ $120000^{12}$.

14 Ce déséquilibre global recouvre des phénomènes différenciés. Il se manifeste à des degrés divers non seulement selon les régions, mais aussi selon les secteurs et les professions. L'artisanat est particulièrement touché : depuis le maximum de 1979 $(40,2 \%)$, sa part dans le système dual est tombée en 1990 à un minimum historique de $33 \%$. Cette évolution a certes un aspect favorable, dans la mesure où, après des années d'offre insuffisante, les jeunes ont de nouveau le choix et peuvent légitimement refuser d'aller dans les entreprises dont la formation est insuffisante. Mais au delà du retournement conjoncturel, apparaît un facteur plus profond, qui touche à la nature des choix professionnels: c'est la désaffection croissante pour les professions manuelles et techniques, ainsi que pour les activités commerciales peu qualifiées au profit des activités de service de moyen et haut niveau et, de façon générale, des métiers administratifs et de bureau.

15 Certes, parallèlement à l'augmentation du nombre de jeunes atteignant le niveau bac (environ $30 \%$ des sorties du système scolaire et $35 \%$ des classes d'âge scolarisées correspondantes $\left.{ }^{13}\right)$, le système dual recrute de plus en plus à ce niveau $(17,2 \%$ des apprentis en 1991). Cette évolution correspond d'ailleurs à l'élévation des qualifications 
requises dans un certain nombre de cas, en particulier par certaines professions commerciales et bancaires, où les produits se complexifient et le contact avec la clientèle devient de plus en plus important, ou encore dans certaines branches du travail des métaux, où des connaissances informatiques deviennent indispensables pour programmer les machines. En recrutant des bacheliers, les employeurs risquent cependant de ne pas amortir leur investissement-formation, car l'apprentissage n'est pour une part croissante de ceux-ci (près de $20 \%$ actuellement) qu'une étape avant la poursuite ultérieure des études.

entreprises peuvent tenter de retenir les jeunes après la fin de leur formation par une politique systématique de formation continue et l'aménagement de plans de carrière attractifs. Mais les possibilités de promotion sont souvent limitées par la configuration hiérarchique. Reste la motivation par le niveau de rémunération, mais celle-ci rencontre des effets de butoir du fait des coûts salariaux déjà très élevés et des classifications en vigueur dans les conventions collectives.

Lorsque la nature des qualifications à transmettre n'exige pas absolument de faire appel à des candidats bacheliers, les employeurs essaient d'échapper à ce risque en recrutant systématiquement des jeunes sortant de la Realschule ou de la Hauptschule, même s'ils n'ont pas obtenu le certificat de fin d'études. Cela signifie que globalement le déficit de recrutement peut finalement profiter à des jeunes jusque-là exclus de l'apprentissage en raison de leur échec scolaire. Encore faut-il que les entreprises en question fassent l'effort nécessaire en comblant les lacunes de formation générale par un renforcement de l'enseignement théorique dans l'établissement et par l'organisation d'un suivi pédagogique beaucoup plus serré. En la matière, la Berufsschule, qui aurait aussi un rôle important à jouer, ne le remplit que très imparfaitement.

18 Pour rétablir les grands équilibres, il faudrait donc 1) attirer davantage de jeunes vers le système dual, 2) faire en sorte que ceux qui y rentrent se tournent aussi vers les professions artisanales ou industrielles, 3) et que s'ils choisissent l'apprentissage dans le tertiaire, ce soit plus souvent pour exercer le métier correspondant que pour continuer immédiatement un parcours de formation visant les études supérieures. Devant l'ampleur des problèmes, tous les acteurs du système dual ont pris conscience de la nécessité d'engager une politique globale.

19 Pour le gouvernement, qui est largement approuvé par les organisations d'employeurs, il faut privilégier deux orientations : faire en sorte que la formation professionnelle ait "la même valeur" (Gleichwertigkeit) que le deuxième cycle d'enseignement général et reconnaître la différenciation des niveaux ${ }^{14}$.

20 En instituant l'"équivalence" du cursus professionnel et du secondaire long, il s'agit d'éviter que l'apprentissage soit perçu comme un cul-de-sac. Sur le plan formel, cela suppose que soit décerné un certificat de fin de formation qui ouvre directement l'accès aux filières de l'enseignement supérieur. Sur le plan des contenus, une adaptation des programmes, notamment l'amélioration de l'enseignement de la Berufsschule, en est la condition nécessaire.

21 Par ailleurs, le gouvernement prône une différenciation officielle des brevets de formation, indépendamment de la hiérarchie implicite qui existe déjà selon les professions et les entreprises choisies. Il souhaite en effet que, à l'intérieur d'un même établissement et pour un même métier, la diversité des capacités et des résultats individuels puisse être prise en compte. En offrant les options qui permettraient 
l'épanouissement des individualités ${ }^{15}$, et en les reconnaissant par des mentions correspondantes, le système dual pourrait espérer retenir les jeunes les plus doués, tout en continuant de former les autres.

Ces propositions sont loin de susciter un accord unanime. L'idée d'une équivalence entre la formation professionnelle et le second cycle d'enseignement général est vue d'un bon oeil par les syndicats et par les professeurs de Berufsschulen. Elle ne suscite pas l'enthousiasme des présidents d'université, qui, loin de croire qu'une telle mesure renforcera l'attrait du système dual, craignent un afflux supplémentaire dans leurs établissements, déjà surchargés.

Mais, le point le plus controversé est le principe de la différenciation, auquel les syndicats sont résolument hostiles ${ }^{16}$. Ils considèrent que les différences de niveau existant entre professions et entreprises sont déjà suffisantes. Ils redoutent que, sous couvert de sauver l'apprentissage, la réforme ne permette la réintroduction systématique de professions semi-qualifiées que l'évolution des règlements de formation depuis la guerre avait pratiquement éliminées. Pour certains experts (comme Burkhardt Lutz), une telle différenciation n'est pas souhaitable pour une raison plus fondamentale, car elle risque d'institutionnaliser au sein même du secteur de l'enseignement professionnel les mécanismes méritocratiques qui nuisent à sa pérennité. Pour les syndicats, il faut chercher la solution dans une formation professionnelle pour tous, implantée dans le second cycle des lycées.

Une telle revendication de cursus intégré formation professionnelle + baccalauréat semble nous ramener à la discussion des années 70. Mais si le débat actuel a effectivement des points communs avec celui d'il y a vingt ans, il s'en distingue sur un aspect fondamental. L'évolution de la théorie sociologique, de l'analyse des besoins de qualification et des modes d'organisation du travail conduit pratiquement tous les acteurs ${ }^{17}$ à reconnaître que les valeurs-clés du professionnalisme et de la polyvalence sont étroitement liées à l'alternance, c'est-à-dire au rôle primordial de l'entreprise dans la formation.

Reste à savoir si le consensus nécessaire à une réforme pourra se dégager et surtout si des aménagements pédagogiques et institutionnels suffiront. Pourra-t-on infléchir les comportements éducatifs de façon significative et durable sans revaloriser le statut de l'ouvrier qualifié, ce qui implique notamment une évolution des hiérarchies de rémunération? Car il en va de la formation professionnelle comme de l'éducation dans son ensemble: on ne peut traiter ses problèmes sans dépasser l'horizon du système éducatif lui-même.

\section{NOTES}

1. Cf. à ce sujet Wolf-Dietrich Greinert, "Hat das duale System der Berufsausbildung seine Zukunft bereits hinter sich ?", in : Berufsbildung n 46 (1992) 2, p. 69-72.

2. Ce terme n'est d'ailleurs apparu qu'en 1964. 
3. Cf. Wolf-Dietrich Greinert, Das duale System der Berufsausbildung in der Bundesrepublik Deutschland, Eschborn, 1992.

4. Cf. à ce sujet Burkhardt Lutz, "Herausforderungen an eine zukunftsorientierte Berufsbildungspolitik", in: Bundesinstitut fur Berufsbildung, Die Rolle der beruflichen Bildung und Berufsbildungsforschung im internationalen Vergleich (colloque du 25/10/1990), Berlin/Bonn, 1991.

5. Cf., en particulier, Horst Kern/Michael Schumann, Industriearbeit und Arbeiterbewu $\beta$ tsein, Francfort s. M., 1970.

6. Les premières analyses en ce sens émanent de Michael J. Piore/Charles F. Sabel, The Second Industrial Divide. Possibilities for Prosperity, New York, 1984, et Horst Kern/Michael Schumann, Das Ende der Arbeitsteilung? Rationalisierung in der industriellen Produktion, Munich, 1984. En français, cf. p. ex. Benjamin Coriat, L'atelier et le robot, Paris, 1990.

7. A cet égard, la réforme des contenus prolonge et approfondit une tendance à la déspécialisation déjà visible à la seule évolution du nombre de règlements d'apprentissage : d'environ 600 en 1970, on est passé aujourd'hui à moins de 400.

8. Nous nous appuyons ici sur la typologie de Dieter Timmermann, "Szenario des Berufsbildungssystems", in: Frank Strikker/Dieter Timmermann (dir.), Berufsausbildung und Arbeitsmarkt in den 90er Jahren, Francfort s. M., 1990.

9. Les filières de promotion vers les emplois de techniciens ont été évoquées au chapitre VI. Pour l'importance significative de la promotion jusqu'aux fonctions de "cadres supérieurs", cf. l'étude de Michel Bauer et Bénédicte Bertin-Mourot, Les viviers des 200 en France et en RFA, (CNRS-Heidrick \& Struggles), Paris, 1991. La présence de dirigeants issus "du rang" fait évidemment bien plus pour la "culture d'entreprise" que tous les discours et toutes les politiques de communication interne.

10. Cf. à ce sujet Hermann Schmidt, "Zukunft von Qualifikation und Bildung”, in : Joachim Jesse et al., Zukunftswissen und Bildungsperspektiven, Baden-Baden, 1988; et Josef Hilbert/Helmut Voelzkow, "Neue theoretische Analysen des Berufsbildungssystems", in: F. Strikker/D. Timmermann (dir.), Berufsbildung und Arbeitsmarkt..., op. cit.

11. Cf. p. ex. Rainer Janisch, "Zur Gefahr einer Privatisierung der beruflichen Erstausbildung in der Bundesrepublik Deutschland", in : arbeiten und lernen, 8/1989, et Wolf-Dieter Greinert, "Auf dem Wege zum Marktmodell? - Bemerkungen zur heraufziehenden Krise der dualen Berufsausbildung in der Bundesrepublik", in : Klaus Harney/Gunter Pätzold, Arbeit und Ausbildung, Wissenschaft und Politik, Francfort s. M., 1990. Sur la situation de l'école professionnelle, cf. p. ex. Heinz-Peter Hahlhege, "Berufsschule und Berufsausbildung: Einseitige Anpassung oder wechselseitige Ergänzung ?”, in : F. Strikker/D. Timmermann, Berufsausbildung..., op. cit.

12. Bundesministerium für Bildung und Wissenschaft, Berufsbildungsbericht 1992, Bad Honnef, 1992, p. 18.

13. Ces chiffres - et les suivants - ont été calculés d'après Bundesministerium für Bildung und Wissenschaft, Grund- und Strukturdaten 1992/93, Bad Honnef, 1992.

14. Pour le point de vue gouvernemental, cf. Fritz Schaumann, "Die Zukunft des dualen Systems", in: Berufsbildung in Wissenschaft und Praxis 1/1991, et "Experten: Ernstmachen mit Gleichwertigkeit der Berufsbildung", in: Handelsblatt 10/12/1992; pour celui du patronat, "Differenzierung, Durchlässigkeit, Leistung. Bildungspolitische Position der Spitzenverbände der Wirtschaft", in : Die berufsbildende Schule, mars 1992.

15. Il est question d'élargir la formation aux champs professionnels voisins ou de permettre l'acquisition de qualifications complémentaires, p. ex. de connaissances techniques pour les agents commerciaux et de notions commerciales pour les apprentis dans des métiers techniques. 16. Cf. p. ex. Andreas Gruschka, "Diplom kontra Gesellenbrief", in: Gewerkschaftliche Bildungspolitik, 3/1992.

17. Le pédagogue Karlheinz A. Geissler représente une exception notable. Pour lui, le système dual a son avenir derrière lui parce qu'il est fondé sur un rapport au travail historiquement 
dépassé, que, par référence à Hannah Arendt, il nomme le "paradigme de la fabrication". Il a défendu ses thèses lors d'un congrès organisé en octobre 1990 par le BIBB ("Perspektiven der Weiterentwicklung des Systems der dualen Ausbildung in der Bundesrepublik", in : BIBB, Die Rolle der beruflichen Bildung und Berufsbildungsforschung im internationalen Vergleich, Berlin/Bonn, 1991) et les a publiées sous une forme légèrement remaniée dans Leviathan 1/1991: "Das Duale System der industriellen Berufsaus-bildung hat keine Zukunft". 


\section{Orientation bibliographique}

\section{En français}

Gesa Chomé, La Formation professionnelle en RFA, Paris, CIRAC, 1985.

Adelheid Hege, "Apprentissage et insertion professionnelle en RFA", in: La Revue de l'IRES, $\mathrm{n}^{\circ}$ 3, printemps-été 1990.

Marc Maurice/François Sellier/Jean-Jacques Silvestre, Politique d'éducation et organisation industrielle en France et en Allemagne, Paris, 1982.

Martine Mobus/Patrick Sevestre, "Formation professionnelle et emploi : un lien plus marqué en Allemagne", in : Economie et statistique, n 246-247, sept.-oct. 1991.

Martine Möbus/Eric Verdier (dir.), Le Système de formation professionnelle en RFA. Résultat de recherches françaises et allemandes, Paris, 1992.

Jean-Jacques Silvestre, "La Professionnalisation : l'exemple allemand", in : Pouvoirs, n 30, 1984.

\section{En allemand}

Bundesministerium für Bildung und Wissenschaft, Grund- und Strukturdaten 199./9., Bad Honnef 199. (annuel).

Bundesministerium für Bildung und Wissenschaft, Berufsbildungsbericht 199., Bad Honnef 199. (annuel).

Wolf-Dietrich Greinert, Das duale System der Berufsbildung in der BRD, Struktur und Funktion, Eschborn, 1992.

Wolf-Dietrich Greinert, "Hat das duale System der Berufsausbildung seine Zukunkft bereits hinter sich ?", in : Berufsbildung, n 4, 1992, p. 69-72.

Joachim Münch (dir.), Das berufliche Bildungswesen in der Bundesrepublik Deutschland, Berlin, 1987. Edition en anglais : Vocational Training in the Federal Republic of Germany, $3^{\mathrm{e}}$ édition, Berlin, 1991.

Karlwilhelm Stratmann/Manfred Schlösser, Das duale System der Berufsbildung, eine historische Analyse seiner Reformdebatten, Francfort s. M., 1990.

Wolfgang Streeck et al., Steuerung und Regulierung der beruflichen Bildung. Die Rolle der Sozialparteien in der Ausbildung und beruflichen Weiterbildung in der Bundesrepublik Deutschland, Berlin, 1987. 
Frank Strikker/Dieter Timmermann, Berufsausbildung und Arbeitsmarkt in den 90er Jahren, Francfort s. M., 1990. 


\section{Adresses utiles}

Commission de l'économie allemande pour la formation professionnelle

1 Kuratorium der Deutschen Wirtschaft für Berufsbildung

2 Buschstraße 83

3 D-5300 BONN 1

4 Tél. : (49-228) 212076

\section{Confédération des syndicats allemands}

5 Deutscher Gewerkschaftsbund

6 Hans-Böckler-Straße 39

7 D-4000 DÜSSELDORF 30

8 Tél. : (49-211) 43 01-0

\section{Fédération des chambres de commerce et d'industrie}

9 Deutscher Industrie- und Handelstag

10 Adenauerallee 148

11 D-5300 BONN 1

12 Tél. :(49-228) 104-0

\section{Institut fédéral de formation professionnelle}

13 Bundesinstitut für Berufsbildung

- Fehrbelliner Platz 3 
Ministère fédéral de la Formation et de la Science

Institut de recherche sur le marché du travail rattaché à l'Office fédéral du travail

Centre européen pour le développement de la formation professionnelle

Conférence permanente des ministres de l'Education des Länder (instance de coordination de la politique d'éducation des Länder)

\footnotetext{
$30 \quad$ Standige Konferenz des Kultusminister der Länder der Bundesrepublik Deutsehland

$31 \quad$ Nassestr. 8

32 D-5300 BONN 1

33 Tél. : (49-228) 501-0
} 


\section{Liste des tableaux et figures} 1973-1990

Tableau 1 (p. 25) :

Répartition des apprentis du système dual par secteur d'activité dans l'ancienne RFA

Tableau 2 (p. 27) :

Les dix professions les plus choisies en 1990 par les apprentis masculins et féminins dans l'ancienne RFA

Tableau 3 (p. 50) :

Répartition des apprentis par catégorie d'établissement

Tableau 4 (p. 76) :

Exemples de coût/apprenti (pour l'année 1990)

Tableau 5 (p. 87) :

Position des 17-18 ans vis-à-vis du système éducatif selon la profession du chef de famille en 1989

Tableau 6 (p. 88) :

Scolarisation des 13-14 ans selon la profession du chef de famille en 1989

Tableau 7 (p. 94) :

Population active occupée selon le diplôme le plus élevé obtenu

Tableau 8 (p. 96) :

Niveau de diplôme des ouvriers par niveau de qualification

Figure 1 (p. 24) :

Nombre total d'apprentis dans les Länder occidentaux 1975-1990

Figure 2 (p. 32) :

Les écoles professionnelles en RFA

Figure 3 (p. 54) :

Procédure d'élaboration des règlements de formation

Figure 4 (p. 59) : 
Formation par niveau pour 14 professions du bâtiment

Figure 5 (p. 62) :

Nouvelles professions de la transformation des métaux - Industrie

Figure 6 (p. 63):

Nouvelles professions de l'électronique - Industrie

Figure 7 (p. 66):

Nouvelles professions de la transformation des métaux - Artisanat

Figure 8 (p. 67) :

Nouvelles professions de l'électronique - Artisanat

Figure 9 (p. 84) :

Le système d'enseignement en République fédérale d'Allemagne

Figure 10 (p. 101):

Taux de chômage des groupes dits "à problèmes"

Figure 11 (p. 102) :

Taux de chômage en fonction des niveaux de diplôme (1975-1991)

Figure 12 (p. 110) :

Position vis-à-vis du système éducatif des 15-19 ans - en \% (population résidente) 


\section{Index thématique}

Artisanat : 14, 17, 25, 51-52, 65-67, 73, 77, 90, 93, 98, 105, 111

Atelier d'apprentissage : 15, 51, 108

Ausbildungsberater - chambres consulaires

Ausbildungsordnung - règlement de formation

Ausbildungsquote - taux d'apprentissage

Berufsaufbauschule : 31,32

Berufsbildungsausschu $\beta$ - commission de formation professionnelle

Berufsbildungsgesetz - loi sur la formation professionnelle

Berufsfachschule - école professionnelle à plein temps

Berufsgrundbildungsjahr - formation professionnelle de base

Berufsschule - école professionnelle du système dual

Berufsvorbereitungsjahr : 29

Bundesinstitut für Berufsbildung (BIBB) - Institut fédéral pour la formation professionnelle

Bundesministerium für Bildung und Wissenschaft - ministère fédéral de la Formation et de la Science

Centres de formation inter-entreprises : $38,44,60,71,73,108$

Chambres consulaires : 40, 41-43

Conseillers d'éducation (Ausbildungsberater) : 43

Chômage : 93, 100-103, 107

Cogestion : 16, 18, 19, 21, 39, 42-43, 48, 49, 55-56, 58-69, 107

Commission de formation professionnelle des Länder (Landesausschu $\beta$ ) : 18, 42

Commission de formation professionnelle (Berufsbildungsausschu $\beta$ ) : 18, 19

Conférence permanente des ministres de l'Education des Länder (KMK) : 40, 42, 55-56

Ecole professionnelle à plein temps (Berufsfachschule) : 29, 30, 32, 33, 94

Ecole professionnelle du système dual (Berufsschule) : 16, 18, 30, 40-47, 56, 60, 71, 72, 73, $75,91,109,113$ 
Examens : 41, 42, 52, 90, 91

Fachgymnasium - lycée technique

Fachhochschule : 31, 32, 98

Fachoberschule : 31,32

Fachschule : 31, 32, 97-98

Fédéralisme scolaire : 16, 37, 56

Financement de l'apprentissage : 20, 21, 42, 47, 60, 71-82, 108

Formateurs en entreprise : 38, 41, 43, 46, 52, 74-75, 81, 91

Formation continue : 38,80

Formation professionnelle de base (Berufsgrundbildungsjahr) : 29, 30, 32, 34, 57

Handicapés : 28

Hauptausschufi (BIBB) - Bundesinstitut für Berufsbildung

Hiérarchie dans l'entreprise : 81

Insertion professionnelle : 73, 79-80, 82, 93-103, 107

Institut fédéral de formation professionnelle : $38,40,55,72$

- commission représentative des Länder (Länderausschuß) : 55

- commission centrale (Hauptausschu $\beta$ ) 38, 40, 55

Interruption du contrat d'apprentissage : 92-93

Jeunes en difficulté : 28

Kultusministerkonferenz (Ständige Konferenz der Kultusminister der Länder- KMK) conférence permanente des ministres de l'Education des Länder

Länderausschu $\beta$ - Bundesinstitut für Berufsbildung (BIBB)

Landesausschüsse - commissions de formation professionnelle des Länder

Loi sur la formation professionnelle (de 1969) : 17-19, 40, 57, 91, 105

Lycée technique (Fachgymnasium) : 31, 32

Marché des places d'apprentissage : 20, 21, 28, 34, 100

Ministère fédéral de la Formation et de la Science : 40, 42, 55

Mobilité professionnelle : 48, 97-98, 107

Organisation du travail : 39, 81, 106

Polyvalence : 49, 52, 57-69

Promotion professionnelle : 48, 97-98, 112

Recrutement des apprentis : 46, 89-90, 97-98

- modalités : 89-90

- origine scolaire : 90

Règlement de formation (Ausbildungsordnung) : 18, 25, 40, 45, 49, 52-69, 107

Subsidiarité : 14,39

Syndicats de salariés : $16-18,28,38-41,55,56,61,64,106,113-114$

Système scolaire (de formation générale) : 83-89, 90, 106, 109-111

Taux d'apprentissage : 26, 50-52, 107

Université : 95, 109-110, 112-113 